Portland State University

PDXScholar

1996

\title{
Jitter and Wander Reduction for a SONET DS3 Desynchronizer Using Predictive Fuzzy Control
}

Kevin Blythe Stanton

Portland State University

Follow this and additional works at: https://pdxscholar.library.pdx.edu/open_access_etds

Part of the Electrical and Computer Engineering Commons Let us know how access to this document benefits you.

\section{Recommended Citation}

Stanton, Kevin Blythe, "Jitter and Wander Reduction for a SONET DS3 Desynchronizer Using Predictive Fuzzy Control" (1996). Dissertations and Theses. Paper 1164.

https://doi.org/10.15760/etd.1163

This Dissertation is brought to you for free and open access. It has been accepted for inclusion in Dissertations and Theses by an authorized administrator of PDXScholar. Please contact us if we can make this document more accessible: pdxscholar@pdx.edu. 


\section{JITTER AND WANDER REDUCTION FOR A SONET/DS3 DESYNCHRONIZER USING PREDICTIVE FUZZY CONTROL}

by

\section{KEVIN BLYTHE STANTON}

\section{A dissertation submitted in partial fulfillment of the} requirements of the degree of

\section{DOCTOR OF PHILOSOPHY \\ in \\ ELECTRICAL AND COMPUTER ENGINEERING}

Portland State University

(C) 1996 
UMI Number: 9635657

Copyright 1996 by Stanton, Revin Blythe

All rights reserved.

UMI Microform 9635657

Copyright 1996, by UMI Company. All rights reserved.

This microform edition is protected against unauthorized copying under Title 17, United States Code.

\section{UMI}

300 North Zeeb Road

Ann Arbor, MI 48103 


\section{DISSERTATION APPROVAL}

The abstract and dissertation of Kevin Blythe Stanton for the Doctor of Philosophy in Electrical and Computer Engineering were presented July 17, 1996, and accepted by the dissertation committee and the doctoral program.

\section{COMMITTEE APPROVALS:}
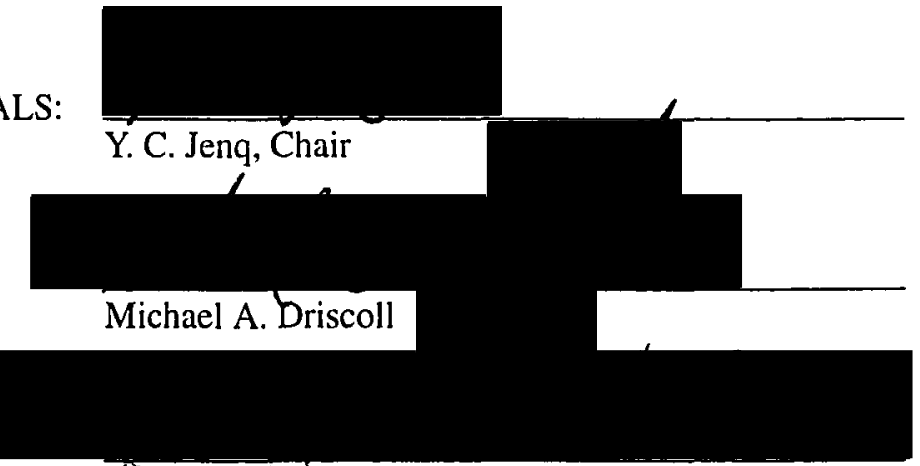

Doughas V/Hall

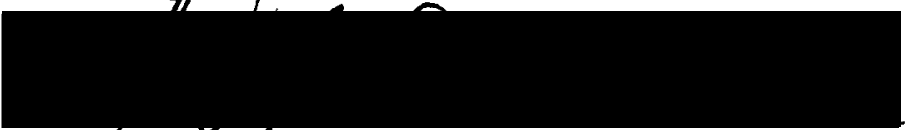

George G. Lendaris

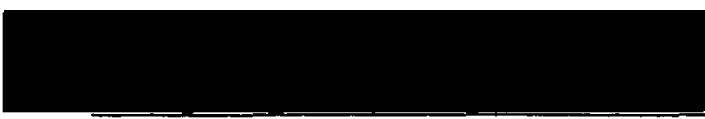

David A. Turcic

Representative of the Office of Graduate Studies

DOCTORAL PROGRAM APPROVAL

Rolf Schaumann, Chair

Department of Electrical Engineering

\section{ACCEPTED FOR PORTLAND STATE UNIVERSITY BY THE LIBRARY}

by on

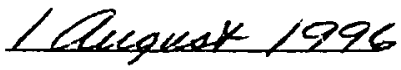




\begin{abstract}
An abstract of the dissertation of Kevin Blythe Stanton for the Doctor of Philosophy in Electrical and Computer Engineering presented July 17, 1996.

Title: Jitter and Wander Reduction for a SONET/DS3 Desynchronizer using Predictive Fuzzy Control
\end{abstract}

Excessive high-frequency jitter or low-frequency wander can create problems within synchronous transmission systems and must be kept within limits to ensure reliable network operation. The emerging Synchronous Optical NETwork (SONET) introduces additional challenges for jitter and wander attenuation equipment (called desynchronizers) when used to carry payloads from the existing Plesiochronous Digital Hierarchy (PDH), such as the DS3. The difficulty is primarily due to the large phase transients resulting from the pointer-based justification technique employed by SONET (called Pointer Justification Events or PJEs).

While some previous desynchronization techniques consider the buffer level in their control actions, none has explicitly considered wander generation. Instead, compliance with jitter, wander, and buffer-size constraints have typically been met implicitly - through testing and tuning of the Phase Locked Loop (PLL) controller.

We investigated a fuzzy/rule-based solution to this desynchronization/constraintsatisfaction problem. But rather than mapping the input state to an action, as is done in standard fuzzy logic, our controller maps a state and a candidate action to a desired result. In other words, this control paradigm employs prediction to evaluate which of a set of candidate actions would result in the "best" predicted performance. Before the 
controller could predict an action's affect on buffer and wander levels, appropriate models were required. The model of the buffer is simply the integral of the frequency difference between the input and output of the PLL, and a novel MTIE Constraint Envelope technique was developed to evaluate future wander performance.

We show that a predictive knowledge-based controller is capable of achieving the following three objectives:

- Reduce jitter implicitly by avoiding unnecessary frequency changes such that the jitter limits specified in relevant standards are met

- Explicitly satisfy both buffer-level and wander (MTIE) constraints by trading off performance in one to meet the hard limit of the other

- When both buffer-level and wander constraints are in danger of violation and cannot be satisfied simultaneously, maintain the preferred constraint by sacrificing the other.

We also show that the computation required for this control algorithm is easily within the reach of modern microprocessors. 


\section{ACKNOWLEDGEMENTS}

First, I would like to thank Coreen. She has been a loving wife, a selfless mother, and a faithful friend. She has never doubted that I could finish whatever I put my mind to. I would also like to thank my mother and father for being a persistent source of encouragement and support. I could not have asked for a better family. A big thanks to my advisor Dr. Y.C. Jenq who was excellent to work with. He made sure that I always had the tools necessary to complete each task and it is doubtful that I would have finished when I did were it not for the guidance and insights he provided each week. I'd like to acknowledge NEC America for funding this research. Specifically, Mr. Brian Reilly and Mr. Steven Gorshe contributed by discussing ideas, offering assistance with T1 standards and documents, and providing their industrial experience. It is doubtful that these results would have been achieved without the freedom they gave me to explore and create. The other members of my committee deserve acknowledgement as well: Dr. Driscoll for his logical/sequential analysis of my research and for telling me early on that to earn a Ph.D. I would "have to really, really want it" (I decided that I did); Dr. Hall for sharing his many practical experiences and insights, time spent on numerous occasions to train me as a teacher, and his convincing arguments for completing my education sooner rather than later; Dr. Lendaris for his thorough and frank evaluation of my ideas and writings, and for a genuinely friendly, personal interest in me and my fam-

ily; Dr. Turcic for his encouragement and flexibility, and for bringing up the question of simulation validity early on so that I had time to consider this critical component. I would like to acknowledge the EE staff and several of my many supportive friends: Tim Grant, Brian Heerwagen, Dan Marvin, Larry Trout, and Will Rogers; my sisters, sistersin-law, brothers-in-law, and parents-in-law for their encouragement; and daughters Tiffany and Chandelle for keeping my spirits up. Finally, I thank my God and Savior for giving me the ability, the opportunity, and the endurance to finish what I started. 


\section{TABLE OF CONTENTS}

PAGE

ACKNOWLEDGEMENTS............................................................................. ii

LIST OF TABLES............................................................................................. vii

LIST OF FIGURES.................................................................................... viii

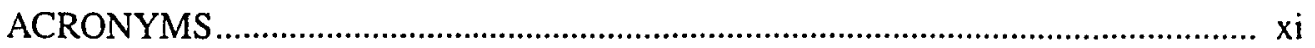

\section{CHAPTER}

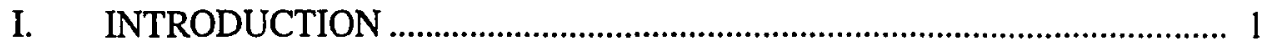

II. SONET/DS3 DESYNCHRONIZATION ................................................. 5

A. DIGITAL COMMUNICATIONS .......................................................... 5

1. Plesiochronous Digital Hierarchy (PDH) ....................................... 6

2. Synchronous Digital Hierarchy (SDH) ........................................... 6

3. Mixing the Hierarchies. ................................................................ 9

B. THE SONET/DS3 DESYNCHRONIZATION PROBLEM .................... 11

1. Basic Architecture ................................................................... 11

2. Sources of Input Jitter .............................................................. 11

3. Desynchronizer Requirements/Constraints .................................. 14

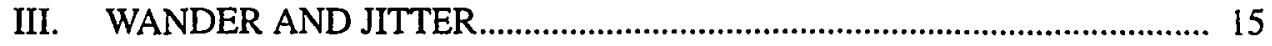

A. WANDER

1. MTIE Definition ........................................................................ 15

2. Wander Filter Design ................................................................ 17

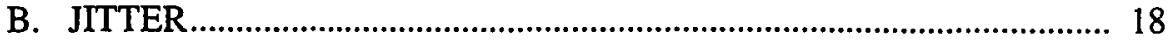

1. Peak-to-Peak Jitter Measurement ............................................... 18

2. Jitter Filter Design ...................................................................... 18

3. Minimum TIE Sample Frequency ............................................ 19 
(iv

C. MTIE CONSTRAINT ENVELOPE .................................................. 23

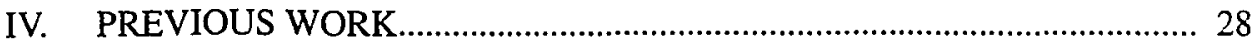

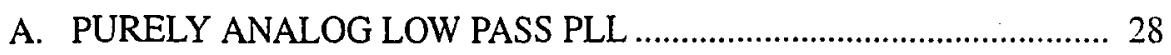

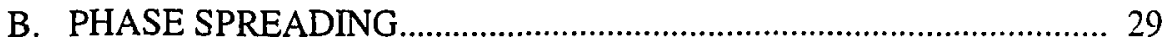

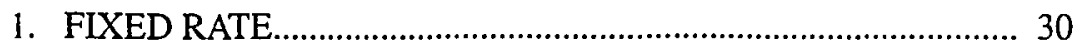

2. VARIABLE RATE................................................................... 30

E. STUFF THRESHOLD MODULATION (STM) ................................. 30

F. ADAPTIVE DITHER BIT LEAKING ................................................ 31

G. FEED FORWARD POINTER SPREADING ...................................... 31

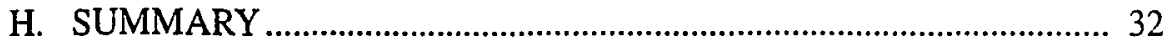

V. THE KNOWLEDGE-BASED CONTROLLER ......................................... 33

A. REASONS FOR CHOOSING AN HEURISTIC APPROACH .............. 34

1. Goal Equations are Nonlinear..................................................... 34

2. Various Modes of Operation ............................................................ 34

3. Opposing Constraints.................................................................. 34

B. THE TRADITIONAL KNOWLEDGE-BASED CONTROLLER .......... 35

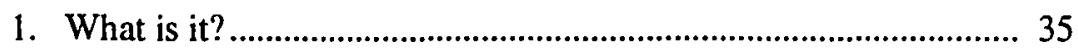

2. When is a Fuzzy Knowledge-Based Controller appropriate?......... 41

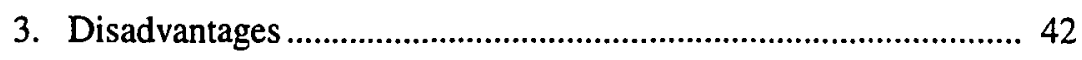

C. PREDICTIVE KNOWLEDGE BASED CONTROLLER ....................... 42

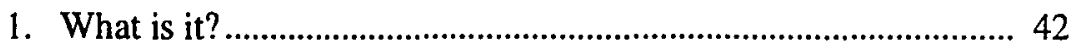

2. When is a PKBC Appropriate....................................................... 47

3. Disadvantages .............................................................................. 47

VI. PKBC FOR THE SONET/DS3 DESYNCHRONIZER …............................. 49

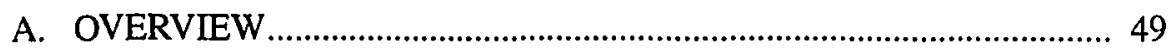

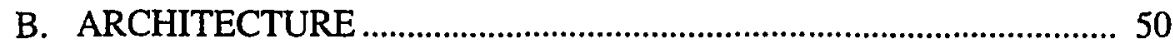




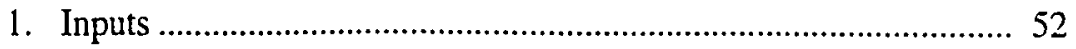

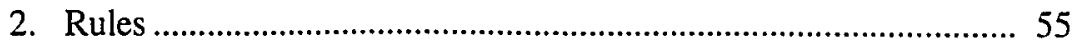

3. Fuzzy Sets .............................................................................. 55

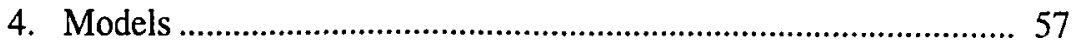

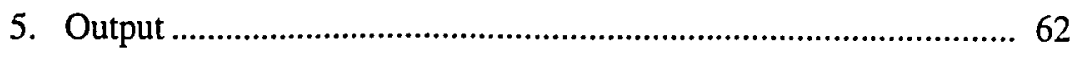

C. STRATEGIES FOR RULE/FUZZY SET DEVELOPMENT .................. 62

1. Partitioning the Space ...................................................................... 63

2. Delta Ruts ……………………………………………………...... 64

3. Full Domain Fuzzy Set Spanning Property .................................... 65

4. Output Fuzzy Sets............................................................................. 67

5. Maintaining MTIE Compliance ………………………………….... 67

6. Maintaining Buffer Compliance ..................................................... 68

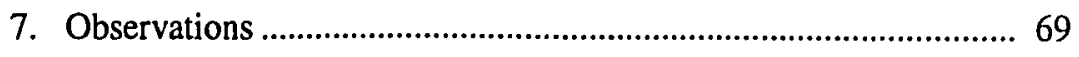

D. FINAL CONTROLLER SPECIFICATION …………………………..... 70

1. FUZZY SETS ……………………………………………....... 71

2. RULES ............................................................................... 72

VII. SIMULATION ENVIRONMENT............................................................ 75

A. SIMULATION FRAMEWORK ……………………………………..... 75

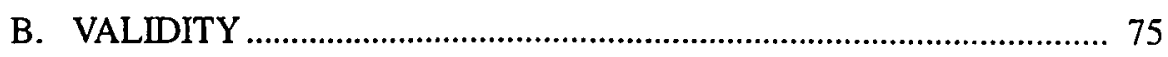

C. SIMULATION MODULES.................................................................. 77

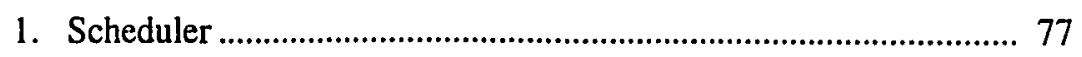

2. Input Signal.................................................................................... 77

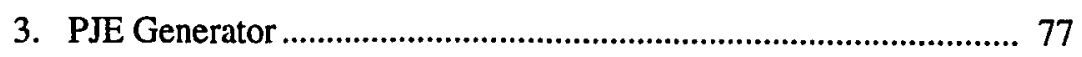

4. DDS Output ................................................................................... 78

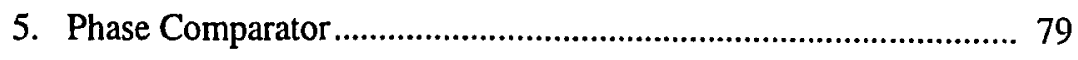

D. SIMULATION MEASUREMENTS ………………………................... 80 
1. TIE Reference Clock ............................................................. 80

2. Jitter Measurement.................................................................. 80

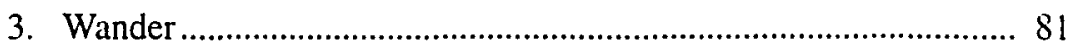

E. GRAPHICAL USER INTERFACE ................................................... 81

F. COMPUTATIONAL COMPLEXITY …............................................ 85

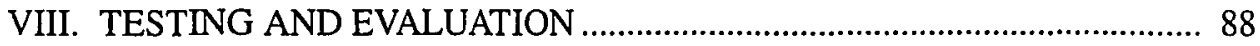

A. STANDARD JITTER TESTS...................................................... 88

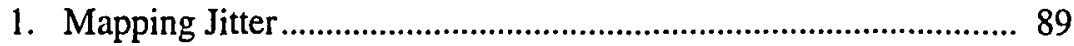

2. Standard Mode ........................................................................... 93

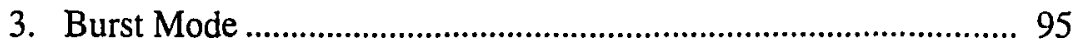

4. Phase-Transient Burst Mode........................................................ 98

5. Degraded Mode .......................................................................... 101

6. Jitter Summary .......................................................................... 111

C. CONSTRAINT-SATISFACTION TESTS .......................................... 111

1. Wander Satisfied ....................................................................... 112

2. Buffer Satisfied ................................................................... 115

IX. CONCLUSIONS/FUTURE WORK ............................................... 117

A. CONCLUSION................................................................................. 117

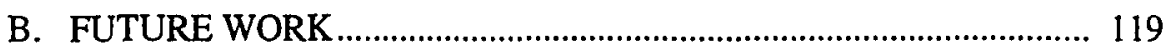




\section{LIST OF TABLES}

TABLE

PAGE

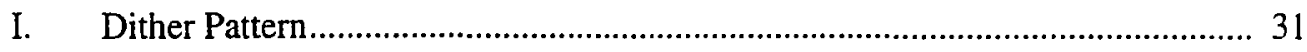

II. Fuzzy Set Definitions .................................................................................. 56

III. Computational Complexity Multipliers ...................................................... 87

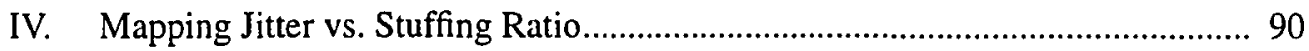

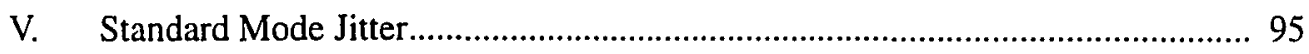

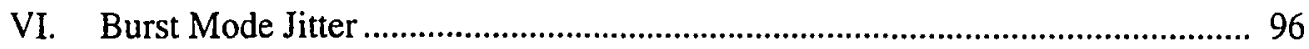

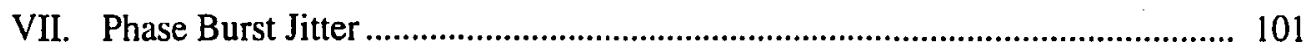

VIII. Degraded Mode (Continuous Pattern) Jitter .................................................. 104

IX. Degraded Mode (87-3 Pattern) Jitter .............................................................. 109

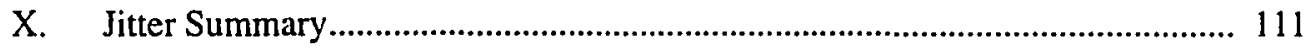

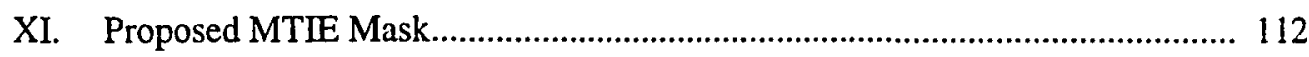




\section{LIST OF FIGURES}

FIGURE

PAGE

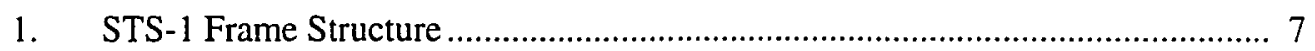

2. SPE Floating Within A Frame

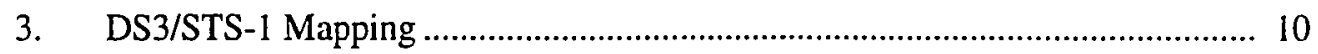

4. SONET/DS3 Desynchronizer...................................................................... 12

5. Illustrated MTIE Definition ....................................................................... 16

6. Percent Error vs. Sample Frequency ........................................................... 22

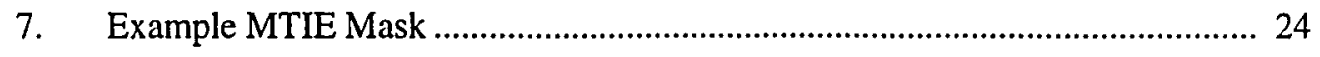

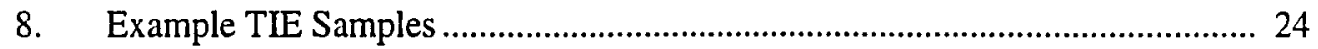

9. Example MTIE Constraint Envelope.............................................................. 25

10. Actual MTIE Constraint Envelope with TIE Projection ................................... 27

11. Architecture for the Phase Spreading technique ............................................. 29

12. Crisp and Fuzzy Definition of "tall" ................................................................ 36

13. Fuzzy Set Definitions for Sample KBC........................................................... 38

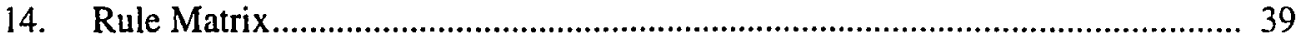

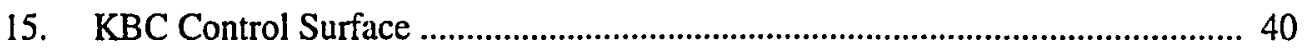

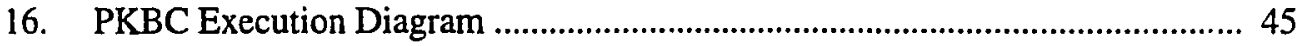

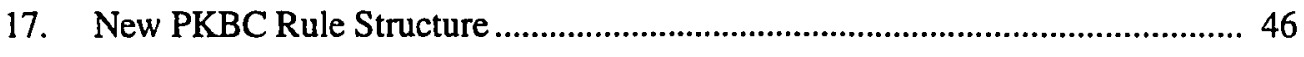

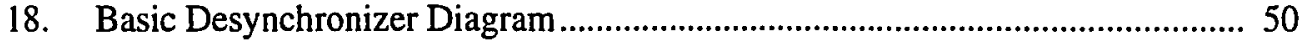

19. PKBC Desynchronizer Controller .................................................................. 51

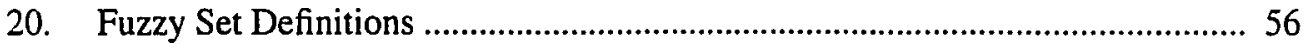

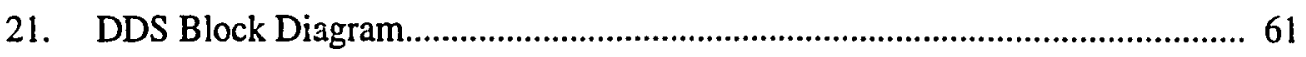

22. Natural and Controlled Vector Fields ................................................................ 64

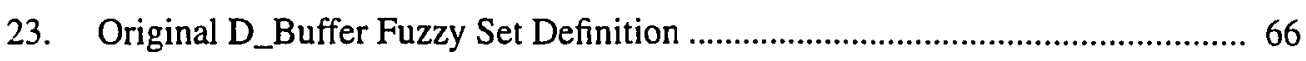


24. Improved (for PKBC) D_Buffer Fuzzy Set Definition........................................ 66

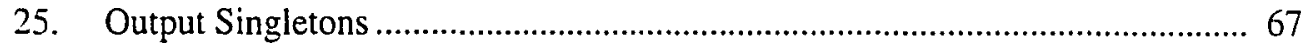

26. Final Buffer Fuzzy Set Definitions ................................................................ 71

27. Final $\Delta \mathrm{Buffer}$ Fuzzy Set Definitions ............................................................ 71

28. Wander Fuzzy Set Definitions ....................................................................... 72

29. Simulator Object Interaction Diagram .......................................................... 76

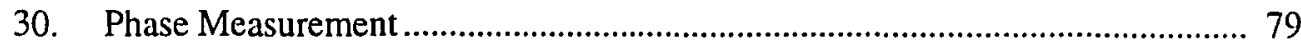

31. Simulation Example Showing Predicted d_buffer movement............................ 83

32. Simulation Example Showing d_buffer movement ............................................. 84

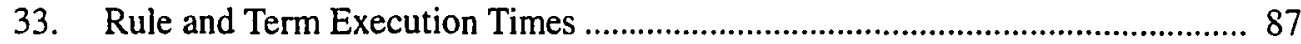

34. Mapping Jitter Simulation Example ................................................................. 91

35. Mapping Jitter vs. Stuffing Ratio.................................................................. 92

36. Standard Mode PJE Test Sequence .................................................................. 93

37. Standard Mode Simulation Example................................................................ 94

38. Standard Mode Jitter.................................................................................... 95

39. Burst Mode PJE Test Sequence ......................................................................... 96

40. Burst Mode Simulation Example ...................................................................... 97

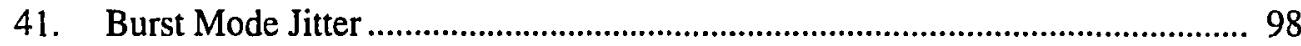

42. Phase-Transient Burst Mode PJE Test Sequence ................................................ 99

43. Phase-Transient Burst Mode Simulation Example......................................... 100

44. Phase-Transient Burst Mode Jitter ...................................................................... 99

45. Degraded Mode PJE Test Sequence (Continuous) ............................................. 102

46. Degraded Mode Simulation (Continuous) Example ........................................ 103

47. Degraded Continuous Pattern Jitter .................................................................... 106

48. Degraded Mode PJE Test Sequence (Continuous) …...................................... 107

49. Degraded Mode Simulation (87-3) Example ..................................................... 108 


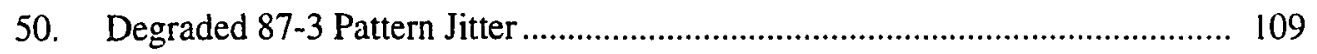

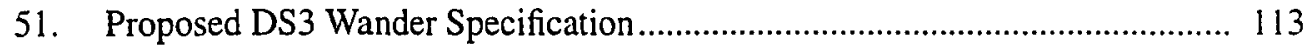

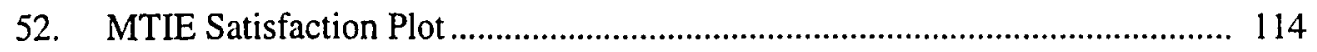

53. MTIE Satisfaction Plot With Buffer........................................................ 115

54. Buffer Satisfaction Plot ....................................................................... 116 


\section{ACRONYMS}

\begin{tabular}{ll}
\hline ATM & Asynchronous Transfer Mode \\
dB & Decibels \\
DDS & Direct Digital Synthesis \\
DS3 & Digital Signal level-3 \\
HPF & High-Pass Filter \\
KBC & Knowledge-Based Controller \\
LPF & Low-Pass Filter \\
MTIE & Maximum Time Interval Error \\
Mbps & Million (Mega)-Bits Per Second \\
MHz & Mega-Hertz \\
NE & Network Element \\
p-p & Peak-to-Peak \\
PDH & Plesiochronous Digital Hierarchy \\
PJE & Pointer Justification Event \\
PKBC & Predictive Knowledge-based Controller \\
PLL & Phase Locked Loop \\
POH & Path OverHead \\
SDH & Synchronous Digital Hierarchy \\
SONET & Synchronous Optical NETwork \\
SPE & Synchronous Payload Envelope \\
STS-1 & Synchronous Transport Signal level-1 \\
SUT & Signal Under Test \\
T3 & Transmission-level term for DS3 \\
TIE & Time Interval Error \\
UI & Unit Interval
\end{tabular}




\section{CHAPTER I}

\section{INTRODUCTION}

The demand for high-speed digital communications is experiencing explosive growth as more people expect more information in less time. The emerging ATM (Asynchronous Transfer Mode) standard promises efficient transfer of diverse types of payload over high-speed channels, and other network technologies are likely to follow. Common to these existing and future services is the need for a physical layer communications link - typically synchronous transmission - and common to all synchronous data transmission systems, is synchronization.

Both the ubiquitous $\mathrm{T} 1$ and $\mathrm{T} 3$ physical layer services and the emerging SONET (Synchronous Optical NETwork) hierarchy employ synchronous data transmission, and thereby require synchronization. In addition, SONET employs global synchronization [1], whereas the Plesiochronous (pronounced "plē sē ä ' krō nas") Digital Hierarchy (PDH), of which the $\mathrm{T} 1$ and $\mathrm{T} 3$ services are members, employs only local synchronization (point to point).

However, when the typically slower PDH services are carried by the faster SONET, the difficult problem of desynchronizing the PDH signal from SONET is introduced. In particular, imperfections in the clock distribution network of any synchronous network topology necessitate realignment of the data payload from time to time to prevent the network element buffers from underflowing or overflowing. SONET implements these justification events by allowing the first byte of each frame of the payload to be advanced or retarded by one byte as needed. However, these so-called justification 
events appear as eight-bit phase transients in the data stream when the PDH payload is reconstructed. Limits on both high-frequency phase transients (jitter) and lowfrequency phase transients (wander) [2] are established by bodies such as ANSI and Bellcore and must be met before equipment can be certified.

Our investigation focused on this - the SONET/DS3 Desynchronization problem. A desynchronizer receives and buffers the "bursty" data to produce a smoother outgoing data rate. Desynchronizers typically consist of an elastic store (buffer) and a Phase Locked Loop (PLL). A PLL, in turn, contains a phase comparator, a loop filter, and a programmable oscillator. If the buffer in the desynchronizer were infinitely large, jitter and wander could be eliminated - at the cost of data latency. If jitter and wander were not a concern, the buffer could be very small - reducing data latency. In practice, however, the buffer has a limited size and jitter and wander must meet specifications. An intelligent controller would use the buffer as much as possible to reduce jitter and wander, yet would not allow it to overflow or underflow. In the event both buffer and wander constraints reach their violation threshold, a decision would be made to maintain one constraint by allowing the other to be violated.

Industry standards [3] specify jitter performance in terms of a set of PJE profiles, applied during testing. Wander specifications [4], however, are universal and apply to any and all possible scenarios. Although current desynchronizer designs exist which satisfy the jitter performance requirements, none explicitly consider wander generation. Our objective was to investigate the ability of a Predictive Knowledge-Based Controller $\mathrm{PKBC}$ to excel in jitter reduction but also, and more importantly, satisfy wander and buffer constraints dynamically.

The PKBC methodology operates on a rulebase ("Knowledge-Based") and uses functional models to choose an action which will result in the highest performance ("Predictive"). Rather than simply specifying condition-action rules, as is the case with 
standard fuzzy logic, the PKBC specifies condition-action-result rules which allow the controller to optimize the "result". In our case, wander and buffer level measures are explicit results, and jitter reduction is implicit in the rules.

We began by investigating the PKBC methodology. We found that an extension of the rule structure allowed us to better express the desired actions of the controller. Then the controller was designed, implemented, and attached to a custom control system simulation environment for evaluation and testing. We found that the PKBC was able to attenuate jitter up to one order of magnitude below the limit and satisfy both wander and buffer constraints when it was possible to do so.

Following is an overview of remaining chapters. In Chapter 2 we explain important concepts needed as background to the desynchronization process, including; PDH, SDH, and the mapping between the two; reasons for PJEs, and their affect on the DS3 payload; and an introduction to jitter and wander. In Chapter 3 we expand the definitions and measurement of jitter (peak-to-peak) and wander (MTIE) and derive the measurement error as a function of sample frequency. We also introduce the MTIE Constraint Envelope, a new development of this work, as well as its efficient calculation. Chapter 4 describes previous work in the area of desynchronization and jitter reduction. The (non-predictive) Knowledge-Based Control paradigm including a section on fuzzy sets and operations on fuzzy sets is described in Chapter 5, along with the extensions necessary to realize a Predictive Knowledge-Based controller (PKBC). Chapter 6 establishes our framework for solving the desynchronization problem with a PKBC and also presents observations and insights gained during the investigation. Simulation is an integral part of this investigation. Thus Chapter 7 is dedicated to the simulator; both its architecture and its implementation. Chapter 8 chronicles the tests that were used to demonstrate the capabilities of the PKBC. Both standard tests of jitter performance and custom tests demonstrating wander and buffer compliance are illustrated, along with 
appropriate graphs and tables to illustrate the simulation results. Finally, Chapter 9 draws conclusions and suggests topics of future work which would build upon our contributions. 


\section{CHAPTER II}

\section{SONET/DS3 DESYNCHRONIZATION}

Before discussing the details of the problem domain in which we frame our investigation, we first provide a brief overview of the field of digital communications. Then, in Section B. we define the specifics of the problem we set out to solve.

\section{A. DIGITAL COMMUNICATIONS}

Common to both digital hierarchies discussed below is the concept of synchronous data transmission. Synchronous transmission means that there is an a priori agreed frequency of transmission between Network Elements (NEs), and thus there is no handshaking between transmitter and receiver. The transmitter blindly sends data at the predetermined data rate. The receiver locks on to the data rate and uses this "recovered" clock to sample the line.

A synchronous hierarchy is a multi-level network with many network elements (NEs) which operate at multiples of the same fundamental frequency and are mutually synchronized. Conversely, an plesiochronous hierarchy is a set of NEs which, while they may communicate synchronously from point to point, are not synchronized to a global frequency reference. The prefix plesio is derived from a greek word meaning "close to", or "near". So, although the levels of the PDH are not strictly synchronous, they are nearly synchronous. 


\section{Plesiochronous Digital Hierarchy (PDH)}

In this hierarchy we have formats and rates commonly known as DS1, DS2, DS3, and so on (alternately $\mathrm{T} 1, \mathrm{~T} 2, \mathrm{~T} 3$ ). When signals which are lower in the hierarchy are combined (multiplexed) to form higher level signals, the incoming bit streams are adaptively padded with "stuff bits" in order to bring them up to a common (higher than nominal) rate before being bit-wise interleaved to form the higher level signal. When demultiplexed, the stuff bits are removed and a desynchronizer is used to smooth the resulting data rate. This is called asynchronous multiplexing, since higher rates are not integer multiples of lower rates.

\section{Synchronous Digital Hierarchy (SDH)}

In contrast to the PDH, all NEs of the SDH (also called the Synchronous Optical NETwork or SONET in North America) operate at a rate traceable to a Stratum-1 global frequency reference (accurate to one part in $1 \times 10^{11}$ ), and higher levels operate at integer multiples of the lower rates. Thus no rate adjustment (stuffing) is required under normal circumstances when multiplexing lower-level signals into a higher-level signal, since the higher rate is synchronized to a multiple of the lower-level signals and both are derived from the same global reference clock. If this were implemented in a perfect environment, our research in this area would be unnecessary. However, imperfections in the clock distribution network occasionally require PJEs for timing justification. PJEs produce jitter and wander as described below.

a. Frame Structure. For historical reasons, SONET is based on a frame-rate of $8 \mathrm{KHz}$ - the bit-rate for a single, digitized telephone line. The frame is organized as nine rows and $3+87 n$ columns, where $\mathrm{n}$ is the level in the hierarchy. The lowest signal in the hierarchy, STS-1 (Synchronous Transport Signal, level 1), operates at a rate of $51.84 \mathrm{MHz}$. All other rates are derived from this rate. Figure 1 illustrates the STS-1 
frame structure.

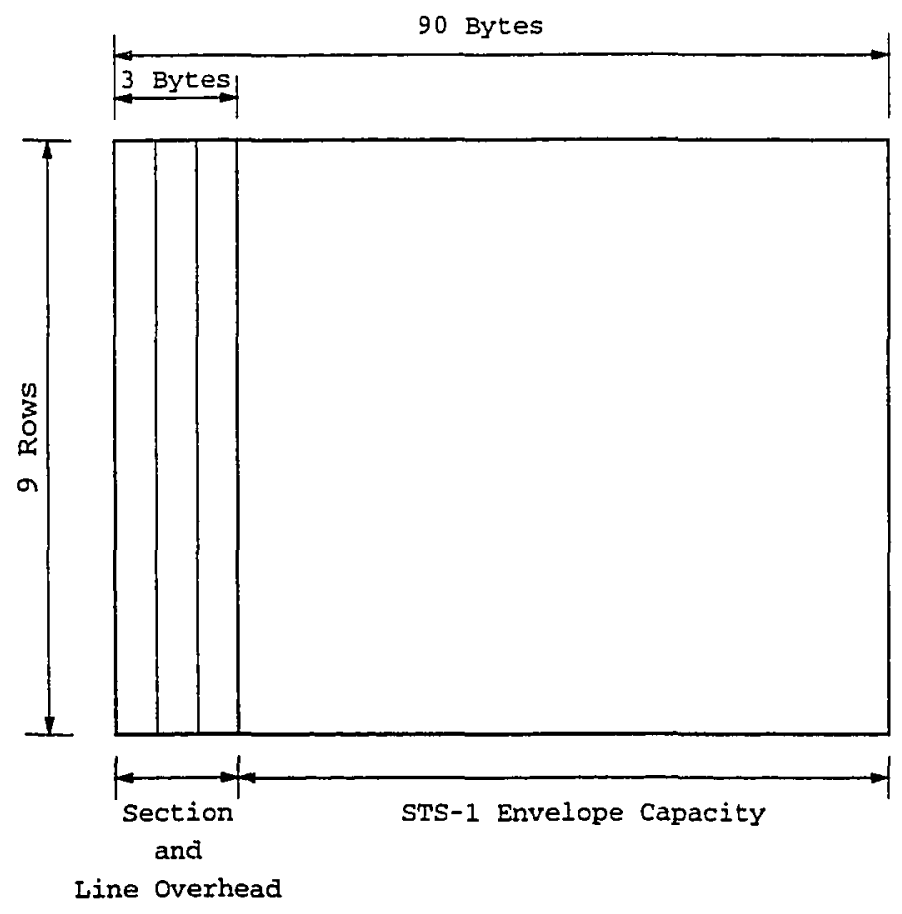

Figure 1. STS-1 Frame Structure

Each row of the frame begins with three bytes of what is called Transport OverHead (TOH). The remaining row capacity is termed the Synchronous Payload Envelope, or SPE. The first byte of the SPE is Path OverHead (POH), leaving $86 n$ bytes for the payload.

b. Pointer Processing. Suppose an NE receives an STS-1, processes it, and retransmits it. If, for reasons described later, the incoming STS-1 data rate were to lag behind the outgoing rate, the network element's transmitting circuit would eventually run out of data, causing an underflow error. Conversely, if the incoming rate were higher than the outgoing rate, the storage buffer would eventually overflow. For this reason, the first byte of the Synchronous Payload Envelope (SPE) is allowed to float 
with respect to the first byte of the frame.

The location of the first byte of the SPE within the frame is indicated by the value of a pointer in the Transport Overhead. If the incoming data rate is higher than the outgoing data rate, a pointer processing module responds by decrementing the "beginningof-envelope" pointer as needed. The result is an advance of subsequent envelopes of data by one byte. Since the first byte of the SPE where the pointer adjustment took effect is the same location in the frame as the last byte of the previous SPE, a special byte of storage is reserved in the overhead for such an occasion. Figure 2 illustrates the floating-SPE concept.

An analogy is drawn in [5] between SONET pointer processing and a man walking through a moving train: "If he moves toward the front, he is moving slightly faster than the train. If he moves toward the rear, he moves slower than the train. A pointer would be a person watching him and always knowing where he is on the train".

Each PJE produces an eight bit phase transient when a signal is extracted (desynchronized) from the SPE. These transients must be smoothed to meet jitter and wander specifications.

This is a difficult problem. First, phase transients resulting from a PJE are eight times larger than those in a PDH network. Also, PJEs may occur at an arbitrarily low frequency and have a long-term nominal rate of zero. A nominal rate of zero has been shown to produce worst-case waiting-time jitter [6].

c. Virtual Tributaries. As the lowest level of the SONET hierarchy, the STS-1 signal can not have other SONET signals as tributaries. Instead, virtual tributaries are defined which allow other payload types to be mapped into the STS-1 frame [7]. The specific mapping is presented in Section 3. 


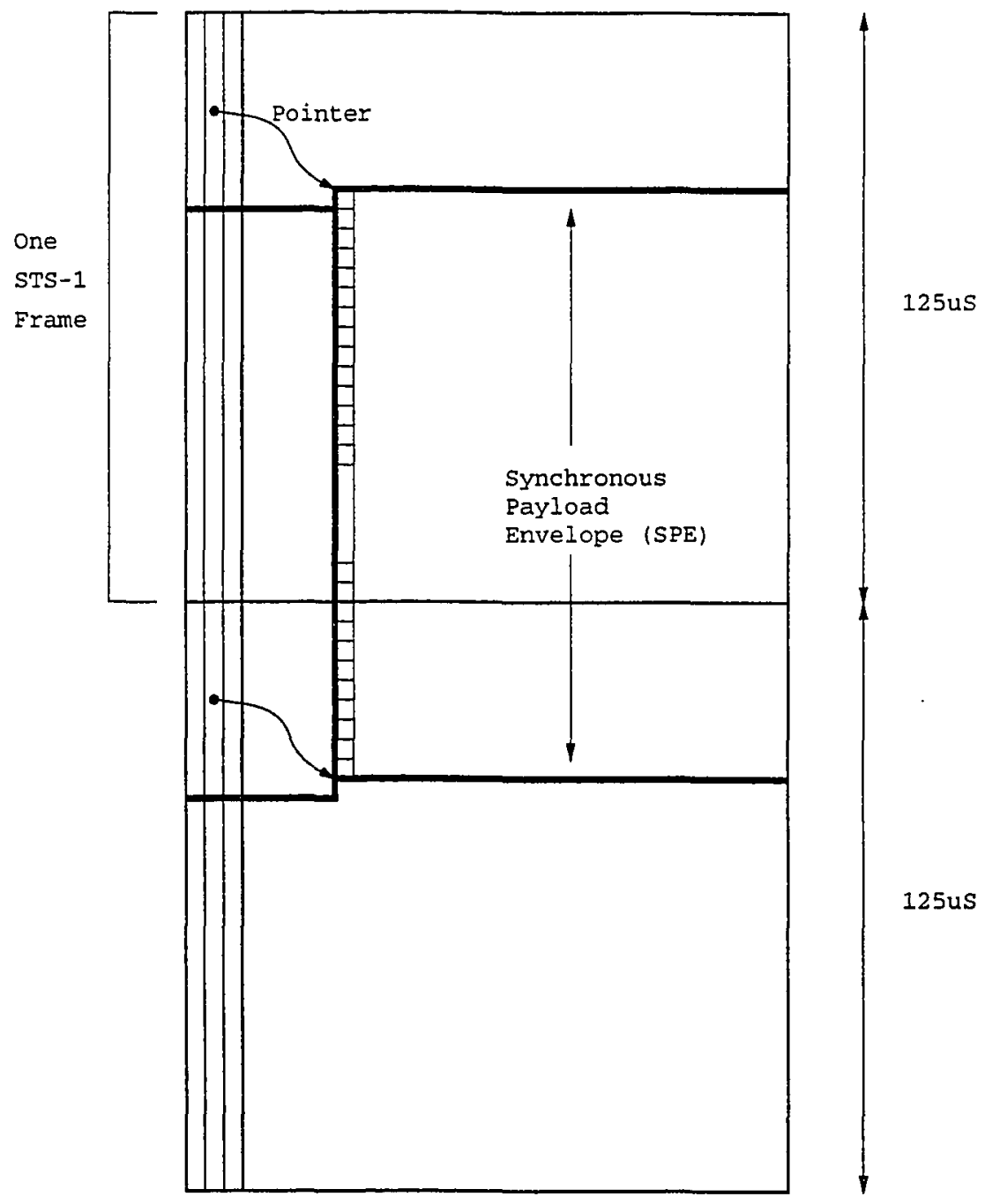

Figure 2. SPE Floating Within A Frame

\section{Mixing the Hierarchies.}

With the growth in demand for commercial DS3 services has come the need for higher and higher public network rates. Converting the DS3 payload into the STS-1 format for transmission within a higher-speed SONET network is an attractive solution. We now look at two primary tasks related with this conversion: mapping, and 
unmapping.

a. Mapping/Synchronization. DS3 operates at a rate of 44.736 Million Bits Per Second (Mbps), and the STS-1 rate is $51.84 \mathrm{Mbps}$. To account for the difference in nominal rate, fixed locations within each SPE row are assigned fixed stuff bits. To account for fluctuations of the DS3 signal with respect to the STS-1 rate, one special stuff bit per row may be assigned payload data as necessary, depending on the relative incoming and outgoing rates. Whether or not this "stuff opportunity" is taken is indicated by a majority vote of the previous Stuff Control (C) bits. It turns out that if incoming and outgoing rates are exactly nominal, the stuff opportunity is taken two thirds of the time. Figure 3 shows the precise location of the Path OverHead (POH), the fixed stuff bits ("R"), the DS3 bits ("I"), the stuff bit ("S"), and the stuff Control bits ("C") [7].

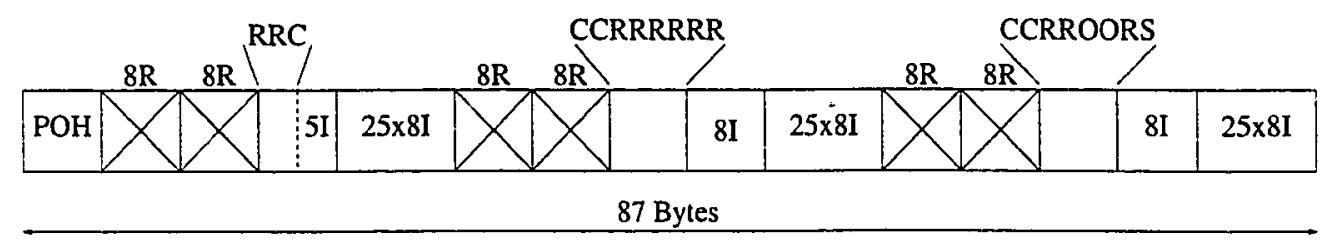

Figure 3. DS3/STS-1 Mapping

b. UnMapping/Desynchronization. Upon arrival at a SONET/PDH mapping node, it is necessary to remove the overhead and fixed stuff bits from the SPE and convert the SONET-mapped DS3 back to its original rate. As the payload data is received, the elastic store write-clock is inhibited during the SONET overhead bytes, fixed stuff bits, and untaken stuff opportunities, since they were not part of the original DS3 payload. The result is a write-clock whose instantaneous rate is that of the STS-1, but with gaps which lower the average rate to the original DS3 level. Thus, after unmapping, the DS3 must be desynchronized - brought back to the rate of the original DS3 signal. To 
accomplish this, the data is stored in a buffer using the gapped clock and read out using the clock generated by the desynchronizer, which is the topic of the next section.

\section{B. THE SONET/DS3 DESYNCHRONIZATION PROBLEM}

\section{Basic Architecture}

A SONET/DS3 desynchronizer is illustrated in Figure 4. The DS3 data is written into the buffer using a gapped clock and the phase comparator measures the phase difference between the gapped write clock and the smooth read clock. The loop filter performs a low-pass operation on these measurements and sends an appropriate command to the programmable oscillator such that future phase difference measurements tend toward some desired value.

The loop filter has the responsibility of both smoothing jitter and wander and preventing buffer overflow - two opposing requirements as we have seen earlier. Past attempts at addressing this challenge are presented in Chapter IV and our approach is presented in Chapter VI. But first, we look more closely at the major causes of jitter in the write (input) clock.

\section{Sources of Input Jitter}

Jitter is related to the variation in the bit arrival time of a data stream. When we speak of jitter caused by the DS3/SONET mapping, we can identify three major sources: mapping jitter, pointer justification events, and bit-stuffing jitter.

a. Mapping Effects. The gaps in the DS3 datastream caused by the removal of SONET overhead and fixed stuff bits are a source of high-frequency jitter which must be suppressed by the desynchronizer. This jitter is constant, since the number of gaps and their location is identical from row to row and frame to frame. 


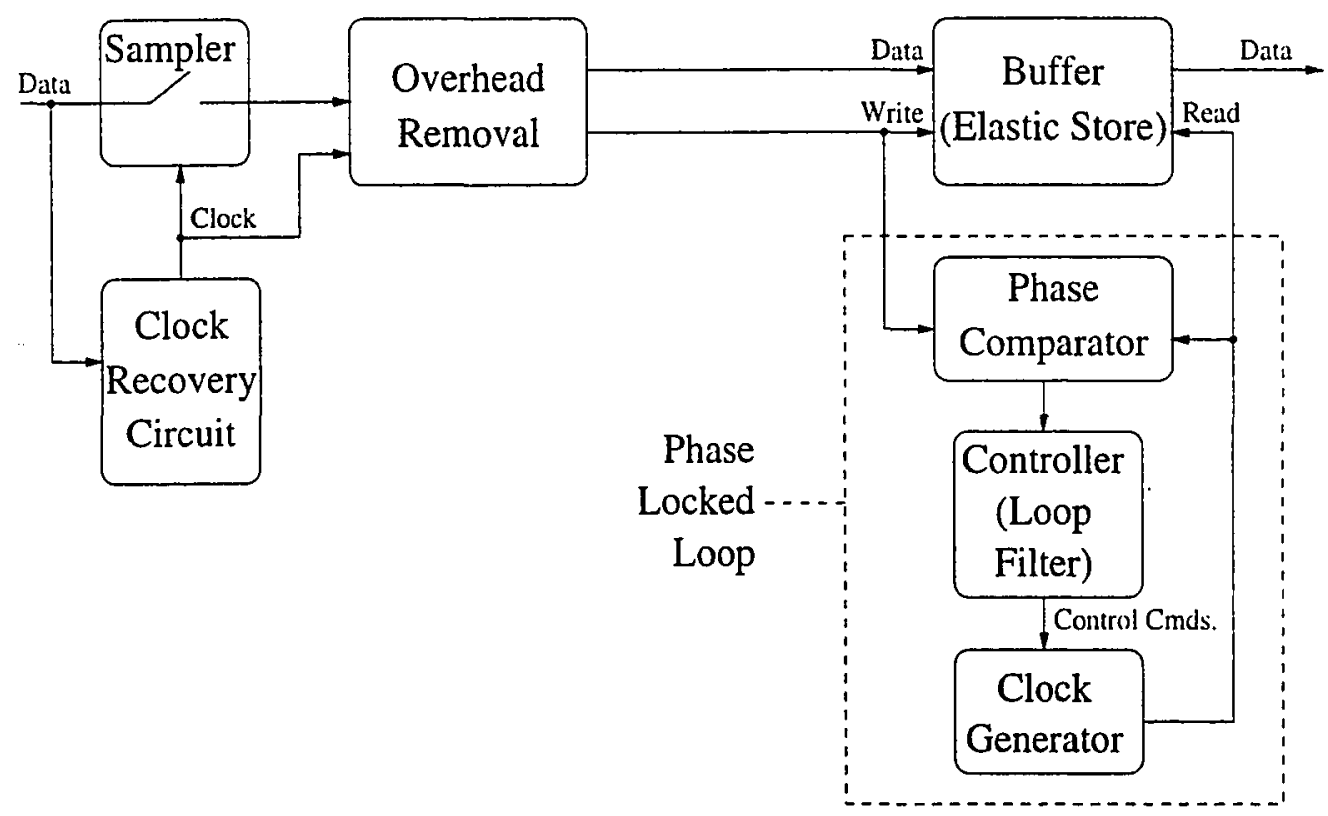

Figure 4. SONET/DS3 Desynchronizer

b. Pointer Justification Events (PJEs). Ideally, pointer adjustments within a SONET network should never be necessary since all network elements are synchronized to the same global reference source. But due to imperfections in the clock distribution network and other problems, pointer adjustments are needed to maintain network synchronization.

As SONET pointer adjustment statistics are observed, four major patterns emerge, which we describe below. The first three occur in spite of traceability to a Stratum-1 reference, and the fourth is due to a loss in Stratum-1 traceability.

Standard Mode. Under normal conditions, occasional PJEs will occur as a result of imperfections in the clock distribution network. Examples include atmospheric changes for airborn signals, fiber length variations and changes in equipment characteristics as temperatures vary, and even the yearly temperature cycle. These PJEs are typically found at intervals of 30 seconds or more. On average, a PJE will eventually be 
canceled by a negative PJE since timing is derived from a common source.

Burst Mode. Under normal conditions, consecutive NEs may be near the threshold for generating a PJE due to reasons described above. A PJE generated at the first NE would then trigger an additional PJE at subsequent NEs, resulting in a sudden burst of PJEs. Although the odds of this happening are not high, a desynchronizer must be capable of attenuating these large, infrequent bursts.

Phase-Transient Burst Mode.

This mode is similar to Burst mode, except that the pointer adjustments are greater in number and arrive at a lower rate.

Degraded Mode. If a NE looses its connection to the clock distribution network due to equipment failure or a broken link, it temporarily switches to a local oscillator. This is called "holdover" mode. Since the required accuracy of the holdover oscillator is much lower than Stratum-1, a frequency offset results. The next NE which is still synchronized to a Stratum-1 would then need to rapidly generate pointer adjustments to resolve the difference. According to tests specified in standards documents, these pointer adjustments may be spaced as closely as 0.034 seconds or as far apart as $10 \mathrm{sec}-$ onds. A desynchronizer must be capable of attenuating the high-frequency jitter resulting from this mode of operation.

c. Bit Stuffing Jitter. To accommodate a range of input DS3 rates, the adaptive bit stuffing mechanism determines on a row-by-row basis whether to include an extra data bit in the stuffing location defined above. The stuffing rate can be derived as

$$
\begin{aligned}
C & =\frac{\text { DS } 3 \text { BitRate }}{\text { RowFrequency }}-D S 3 \text { BitsPerRow } \\
& =\frac{D S 3 \text { BitRate }}{8 K \cdot 9}-621
\end{aligned}
$$

At the nominal DS3 rate of $44.736 \mathrm{Mbs}, C$ equals $1 / 3$. Stuffing jitter is considered a 
"mapping" effect.

\section{Desynchronizer Requirements/Constraints}

We now look at the desynchronizer and constraints it must satisfy.

a. Buffer. Typically, the PLL controller attempts to keep the buffer half-full. If the fill-level is too low, underfiow is threatened. If the fill-level is too high the danger is overflow.

A half-full buffer is said to have a fill-level of zero. Similarly, a buffer which is more than half full is said to have a positive fill-level and a buffer which is less than half-full is said to have a negative fill-level. If an infinite buffer were used, any fixed output frequency less than the input would yield optimal jitter performance. Of course such a requirement cannot be met in practice. In addition, large data buffers increase the data latency of the communications channel, slowing response times.

b. Phase Variation (Jitter and Wander). Both jitter and wander calculations begin with a measure of phase variation, called Time Interval Error (TIE, pronounced " $t \bar{i}$ "). TIE is a measure of the difference in phase between a signal under test and a reference signal,

$$
T I E(t)=\int_{0}^{t} f_{S U T}(t)-f_{R E F} d t
$$

where $f_{S U T}(t)$ is the frequency of the signal under test, and $f_{R E F}$ is the constant reference frequency. TIE is typicaily specified in Unit Intervals, or UI (cycles of the nominal frequency). Both low frequency and high frequency (jitter) phase variation measures are derived from this signal.

The next chapter defines wander and jitter and derives criteria for accurately measuring them in the discrete-time domain. Also, the method for predicting compliance with wander constraints is developed. 


\section{CHAPTER III}

\section{WANDER AND JITTER}

Bounded jitter and wander are necessary for error-free operation of synchronous transmission networks. In this chapter we define "jitter" and "wander" in the context of the PDH hierarchy and discuss established measurement procedures. For a broader treatment of jitter in digital transmission systems the reader is referred to [8].

In Section $\mathrm{C}$, after defining jitter and wander in Sections $\mathrm{A}$ and $\mathrm{B}$, we present a novel technique for transforming past measurements and wander specifications into a constraint envelope which can be used to predict future wander compliance.

\section{A. WANDER}

\section{MTIE Definition}

Maximum Time Interval Error (MTIE, pronounced "em $t \bar{i}$ ") is the primary wander measure. Rather than characterizing wander with a single quantity, MTIE is a function of the width of an observation window in which a peak-to-peak measurement is taken, and is expressed as a curve. Essentially, MTIE is a time-independent measure of long-term drift. Thus it is logical to first pass the TIE measurements through a low-pass filter. But before deriving the filter equation, we first illustrate the MTIE calculation itself.

We define $X(t)$ to be the filtered TIE samples, of which $N$ samples have been taken at an interval of $t_{0}$ seconds. Figure 5 illustrates the MTIE calculation. First, a peak-to-peak calculation is performed within a window (observation period) of size $S$. 


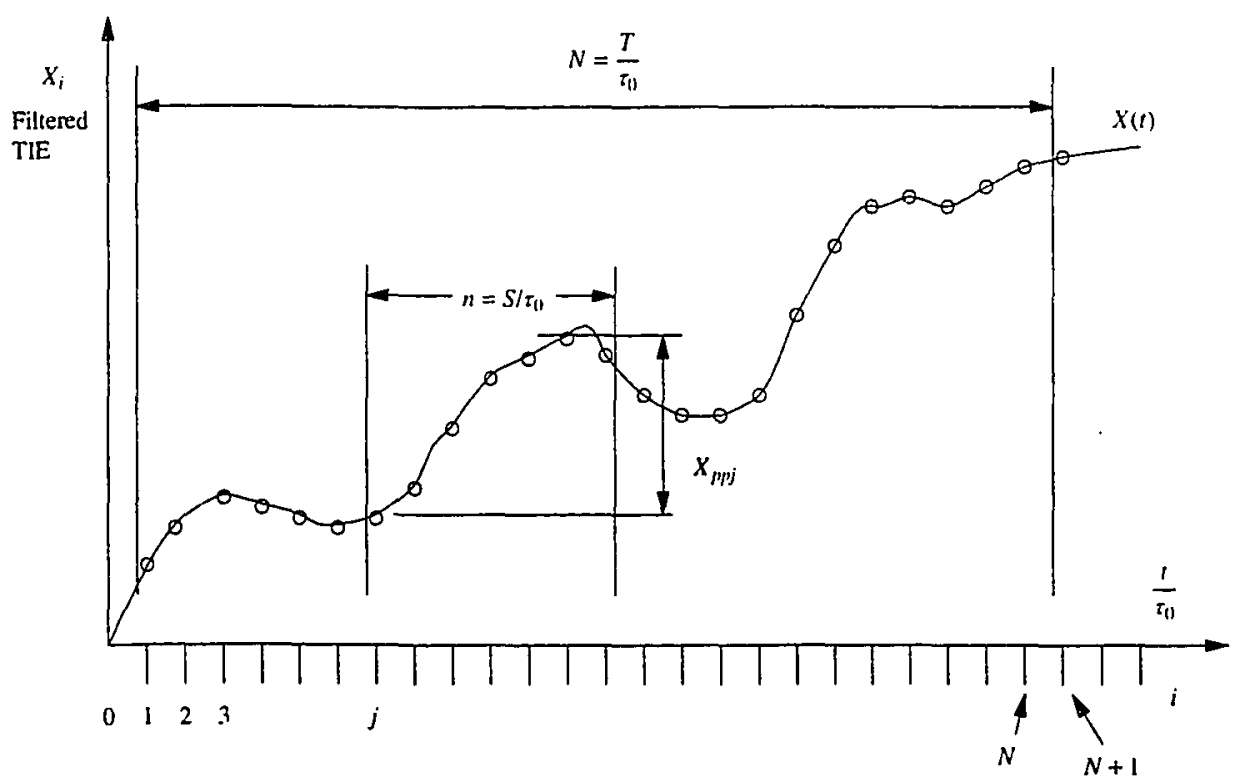

Figure 5. Illustrated MTIE Definition

Then, the largest peak-to-peak value for all such windows of size $S$ within the $N$ samples becomes the MTIE value for that observation period. Window sizes from 2 to $N$ are used, resulting in an MTIE curve.

Standards documents specify an MTIE mask, under which the measured MTIE curve must fall. This measurement is then used to test MTEE compliance. Our goal is to ensure MTIE compliance.

In order to ensure compliance, we developed a novel algorithm for combining past TIE samples and the MTIE mask to determine the limits of future TIE samples, and thereby restrict the actions of the controller in real-time to those which conform to the MTIE mask. This so-called MTIE Constraint Envelope is discussed in Section C. 


\section{Wander Filter Design}

ANSI specifications [2] require the TIE signal to be passed through a single-pole low-pass filter with a $10 \mathrm{~Hz}, 3 \mathrm{~dB}$ cutoff before performing the MTIE calculation. Next we briefly describe the design of a digital filter with these characteristics.

The expression for the discrete-time low-pass filter may be derived from a standard continuous-time, single-pole low-pass filter,

$$
H_{L P}(s)=\frac{\alpha}{s+\alpha}
$$

with an appropriate substitution for $s$ using the bilinear transformation,

$$
H_{L P}(z)=\left.H_{L P}(s)\right|_{s=\frac{1-z^{-1}}{1+z^{-1}}}
$$

$\alpha$ must be pre-warped because of the nonlinearity of the bilinear transformation [9], based on the cutoff frequency $f_{c}$

$$
\alpha=\tan \left(\frac{\pi f_{c}}{f_{s}}\right)
$$

To simplify calculations, we define $a$ and $b$ as,

$$
a=\frac{1-\alpha}{1+\alpha}
$$

and

$$
b=\frac{\alpha}{1+\alpha}
$$

The transfer function in the $\mathrm{z}$-domain then becomes,

$$
H_{L P}(z)=\frac{b+b z^{-1}}{1-a z^{-1}}
$$

This expression may be realized by an Infinite Impulse Response (IIR) filter with the following difference equation, 


$$
y_{L P}[n]=b x[n]+b x[n-1]+a y_{L P}[n-1]
$$

where $x[]$ is the sequence of raw TIE samples and $y_{L^{P}}[]$ is the filtered result.

\section{B. JITTER}

\section{Peak-to-Peak Jitter Measurement}

Jitter is a measurement of high-frequency TIE variations. Unlike wander. which is expressed as a curve, jitter is represented by a single value.

Once TIE measurement is finished (the simulation is over), the peak-to-peak (p-p) value of the filtered TIE samples is used to represent total jitter. This is analogous to a single MTIE calculation with an observation period $S$ equal to the entire measurement period $(N)$.

Although ANSI specifications places restrictions on p-p jitter, the limit is different for each test scenario (see Chapter VIII), and therefore cannot be used as a global constraint in the same sense that MTIE is used as a constraint.

Nevertheless, p-p jitter is an important measure of a desynchronizer's performance and is often used by equipment manufactures to represent the performance of a desynchronizer.

\section{Jitter Filter Design}

The jitter signal is generated by passing TIE samples through a single-pole, highpass filter with a $10 \mathrm{~Hz}, 3 \mathrm{~dB}$ cutoff frequency [2].

In the discrete-time domain, the filter may be derived as

$$
H_{H P}(z)=\left.H_{H P}(s)\right|_{s=\frac{1-z^{-1}}{1+a^{-1}}} \frac{s}{s+\alpha}
$$


Since the low-pass wander filter and high-pass jitter filter have the same cutoff frequency, we can use an equivalence relation to derive the expression of one from the other. Specifically,

$$
\begin{aligned}
H_{l p p} & =1-H_{l p} \\
& =\frac{(1-b)-(a+b) z^{-1}}{1-a z^{-1}}
\end{aligned}
$$

which yields the difference equation:

$$
y_{H P}[n]=(1-b) x[n]-(a+b) x[n-1]+a y_{H P}[n-1]
$$

where $x[]$ is the sequence of raw TIE samples and $y_{H P}[]$ is the filtered result.

\section{Minimum TIE Sample Frequency}

Since MTIE considers only low-frequency components of the TIE signal, a TIE sample rate which is several multiples of the cutoff frequency is adequate. Jitter, on the other hand, requires a filtered TIE signal with frequency components which extend to infinity. Unfortunately, a discrete-time system such as ours is not able to take sample fast enough to meet this requirement. Fortunately, however, we can calculate the error which results from a non-infinite sample frequency. Once this expression is know, choosing the sample rate is reduced to specifying an allowable error and finding the corresponding sample frequency. In the following we assume that output jitter results only from frequency steps, which is true for a controller employing a DDS (Direct Digital Synthesis, described in Section 4.E of Chapter VI) for frequency generation.

First we derive an exact expression for jitter resulting from a frequency step, and then for the jitter measured by a discrete-time system. Comparison of these two results gives us the percent error as a function of sample period. 
a. Continuous-Time Jitter Calculation. Jitter is a peak-to-peak measure of the filtered integral of a frequency step. The integral of a step function is a ramp, with slope equal to the magnitude of the step (mag in $\mathrm{Hz}$ ). Thus the jitter signal $J(s)$ is

$$
H_{j}(s)=\operatorname{mag} \frac{s}{s\left(s+2 \pi f_{c}\right)}
$$

where $f_{c}$ is the cutoff frequency of the high-pass filter in Hz. The inverse Laplace transform yields the time-domain jitter signal

$$
j(t)=\frac{m a g}{2 \pi f_{c}}\left(1-e^{-2 \pi f_{\mathrm{c}} t}\right)
$$

Since the expression is monotonically increasing, its peak-to-peak measurement is equal to the difference between its initial and final values

$$
\underset{t \rightarrow 0}{j(t)}=\frac{m a g}{2 \pi f_{c}}\left(1-e^{-2 \pi f_{c} 0}\right)=0
$$

and

$$
\underset{t \rightarrow \infty}{j(t)}=\frac{m a g}{2 \pi f_{c}}\left(1-e^{-2 \pi f_{c} \infty}\right)=\frac{m a g}{2 \pi f_{c}}
$$

Thus tine jitter resulting from a frequency step of magnitude mag is

$$
\text { jitter }(\mathrm{p}-\mathrm{p})_{\text {continuous }}=\frac{m a g}{2 \pi f_{c}}
$$

This implies that with a cutoff frequency $f_{c}$ of $10 \mathrm{~Hz}$, one UI of jitter is produced by a frequency step of $2 \pi f_{c} \approx 62.8 \mathrm{~Hz}$

b. Discrete-Time Jitter Calculation. In the discrete-time domain, a ramp with slope mag multiplied by the high-pass filter designed earlier yields

$$
J(z)=\operatorname{mag} T_{s} \frac{z^{-1}}{(1+\alpha)-2 z^{-1}+(1-\alpha) z^{-2}}
$$

where the frequency variable $\alpha$ must now be pre-warped using the bilinear 
transformation

$$
\alpha=\tan \left(\frac{\pi f_{c}}{f_{s}}\right)
$$

As before, we derive the resulting peak-to-peak jitter measure by finding the initial and final values of the jitter signal, this time by applying initial and final value theorems. The initial value is

$$
\begin{aligned}
\lim _{t \rightarrow 0} j(t) & =\lim _{z \rightarrow \infty} J(z) \\
& =0
\end{aligned}
$$

and the final value is

$$
\begin{aligned}
\lim _{t \rightarrow \infty} j(t) & =\lim _{z \rightarrow 1}\left(1-z^{-1}\right) J(z) \\
& =\frac{m a g}{2} \frac{1}{\tan \left(f_{c} \frac{\pi}{f_{s}}\right) f_{s}}
\end{aligned}
$$

Peak-to-peak jitter is the difference between the two limits;

$$
\operatorname{jitter}(\mathrm{p}-\mathrm{p})_{\text {discrete }}=\frac{m a g}{2} \frac{1}{\tan \left(f_{c} \frac{\pi}{f_{s}}\right) f_{s}}
$$

For validation, we verified that the discrete-time jitter expression converged to the continuous-time jitter expression as the sample frequency approached infinity.

$$
\lim _{f_{s} \rightarrow \infty} \frac{m a g}{2} \frac{1}{\tan \left(f_{c} \frac{\pi}{f_{s}}\right) f_{s}}=\frac{m a g}{2 \pi f_{c}}=\operatorname{jitter}(\mathrm{p}-\mathrm{p})_{\text {continuous }}
$$


c. Jitter Measurement Error. Combining the results from the previous two sections we arrive at an expression for the jitter measurement error resulting from sampling TIE at a rate less than infinity,

$$
\operatorname{error}(\%)=100\left(1-\frac{f_{c} \pi}{\tan \left(\frac{f_{c} \pi}{f_{s}}\right) f_{s}}\right)
$$

Although a closed-form expression for the sample frequency as a function of error percentage does not exist, the above expression decreases monotonically above the singularity at $f_{c}$, so numerical solutions are easily found. This may be seen in Figure 6 .

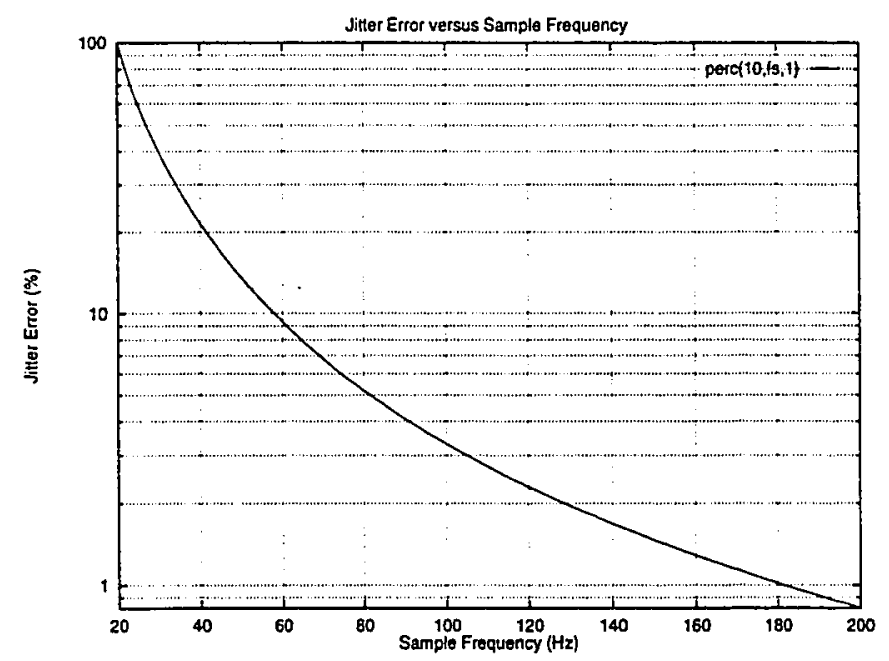

\section{Figure 6. Percent Error vs. Sample Frequency}

Notice that the error, in percent, is independent of the magnitude of the frequency step mag. This property increases the utility of this result since, although the controller adjusts the frequency with steps of varying magnitudes, the measurement error remains constant. 
Suppose an error of $1 \%$ were deemed acceptable. Equation (1) states that a TIE sample frequency of $200 \mathrm{~Hz}$ would suffice, yielding an error of only $0.82 \%$.

\section{MTIE CONSTRAINT ENVELOPE}

One contribution of our research is the development of an MTIE Constraint Envelope. The MTIE calculation is defined for a set of low-pass filtered TIE samples. If the MTIE mask is to be used as feedback to the control algorithm, some method is needed to determine the constraints on future TIE samples. Given a mask defining MTIE over observation periods from $S_{0}$ to $S_{n}$, a set of previous TIE samples TIE[i] and a proposed frequency offset $f_{\text {offset }}$ from the nominal frequency, the question may be asked: At what time in the future will the mask be violated?

Following are expressions for the upper and lower bound on future TIE samples. Any future TIE sample which lies above the upper boundary or below the lower boundary will necessarily result in a violation of MTIE.

$$
\begin{aligned}
& \operatorname{upper}[p]=\min _{i=0}^{N_{\text {maxk }}-p}\left[\min _{j=0}^{i}(T I E[j]+\operatorname{mask}(j+p))\right] \\
& \operatorname{lower}[p]=\max _{i=0}^{N_{\text {maxk }}-p}\left[\max _{j=0}^{i}(T I E[j]-\operatorname{mask}(j+p))\right]
\end{aligned}
$$

where $p$ is the envelope index, with $p=1$ as the first envelope point. $T I E[i]$ refers to previous TIE samples with $i=0$ indicating the current sample and $i=1$ the previous TIE sample. $N_{\text {mask }}$ is the maximum observation period of the mask, in units of TIE sample periods, and mask $(j+p)$ is the MTIE mask value for an observation time of $(j+p) T_{T I E}$ where $T_{T I E}$ is the TIE sample period.

We illustrate the calculation of the MTIE constraint envelope with a simple example. We will use the mask shown in Figure 7 and the TIE samples (taken at an interval 


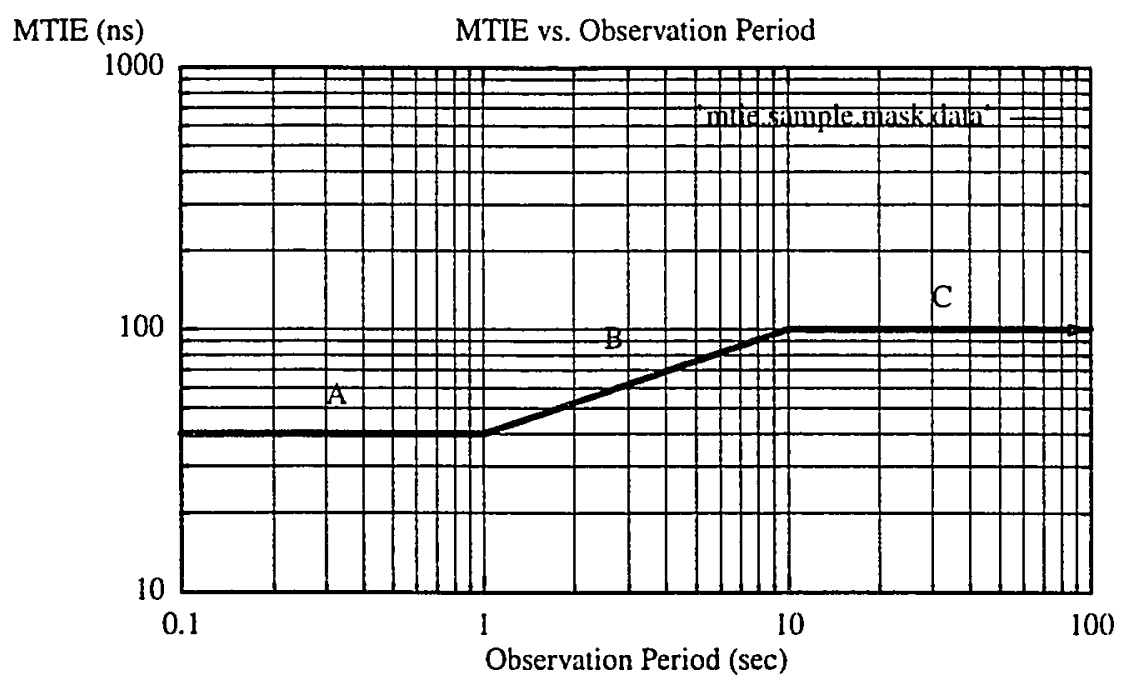

Figure 7. Example MTIE Mask

of $S_{0}=0.1 \mathrm{~s}$ ) shown in Figure 8 .

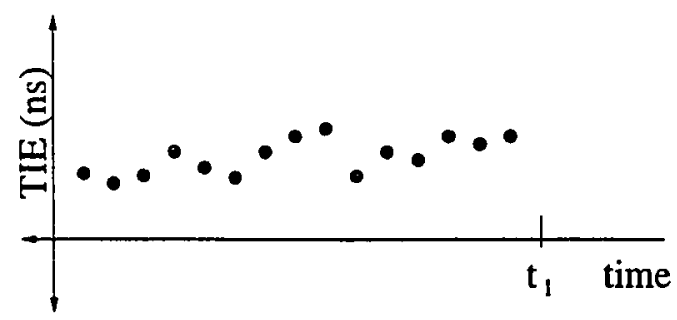

Figure 8. Example TIE Samples

The question is: What values of TIE for the next sample period $\left(t_{1}\right)$ will satisfy the MTIE mask? To answer the question, one must look at the most recent sample, add and subtract the MTIE value for an observation period of $S_{0}$, and plot the two points to form an upper and lower bound. Next, the maximum and minimum of the two most recent samples are calculated and the corresponding mask value for an observation period of $2 \cdot S_{0}$ is subtracted and added, respectively. This process continues until either 
all TIE samples are exhausted or the upper limit of the observation period defined by the mask is reached. The smallest upper bound and the largest lower bound values become the first envelope pair.

Next, the above process is repeated, beginning with the current TIE, but this time using an observation period of $2 \cdot S_{0}$. The result is the second envelope pair. The envelope is generated in this way, and terminates when either the mask is exhausted or the desired length of the envelope is reached. Figure 9 illustrates the envelope which would result from this example.

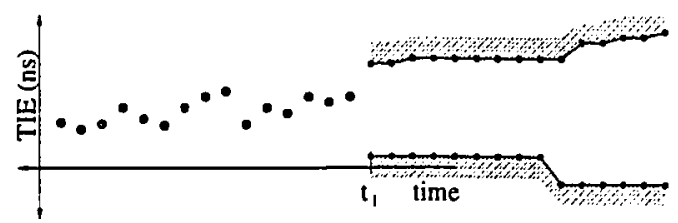

Figure 9. Example MTIE Constraint Envelope

After the envelope is generated, the projected TIE plot from a proposed offset from the nominal frequency ( $f_{\text {offset }}$ ) is plotted with the envelope

$$
T I E\left[n \cdot T_{T I E}\right]=T I E[0]+f_{\text {offset }} t\left(-n \cdot T_{T I E}\right)
$$

where the index $n$ is negative, since it is indexing into the future.

Finally, a search is performed to find the point (if it exists) where the above TIE projection crosses either the upper or lower boundaries of the envelope. The crossing, $n_{\text {viol }}$, indicates the time where the proposed frequency offset would result in an MTIE violation. This quantity is used by the controller to evaluate the "goodness" of the proposed offset.

The computational complexity [10] of the envelope generation increases as $O\left(n_{\text {mask }} \cdot n_{\text {env }}\right)$ where $n_{\text {mask }}$ is the length of the mask and $n_{e n v}$ is the desired length of the 
envelope, both in units of $T_{T I E}$. This complexity may be understood by realizing that it is necessary to consider each observation period of the mask for each previous TIE sample. If, for example, the mask extends to $5000 \mathrm{~s}, T_{T I E}$ is $0.1 \mathrm{~s}$, and the maximum number of envelope points is desired, the above calculation would require storage of 50,000 past TIE samples, and more than $50,000 \cdot 5000$ total iterations of the loop. This calculation, carried out repeatedly (one per TIE sample period) would soon dominate the total computational load of the algorithm.

So instead of generating the envelope in linear time $(p=n T)$, we modified the increment of $p$ to increase exponentially $\left(p=b^{n T}\right)$. The parameter $b$ determines the growth rate of the index, and the computational complexity of the algorithm is thus reduced to $O\left(n_{\text {musk }} \log _{b} n_{e n v}\right)$. The resulting computational load is minimal compared to the rest of the simulator for $b>1.5$.

The loss of resolution is not a concern, since the more important, close envelope points are calculated with better resolution than envelope points which are far away. As an added feature, when the crossing point $n_{\text {viol }}$ falls between two envelope points (which is nearly always the case), interpolation is used to estimate the violation time more precisely.

Figure 10 shows a representative MTIE constraint envelope and the projected TIE resulting from a proposed frequency offset in a real simulation. From the Figure we see that the proposed frequency offset would violate MTIE in about 600 sample times. 


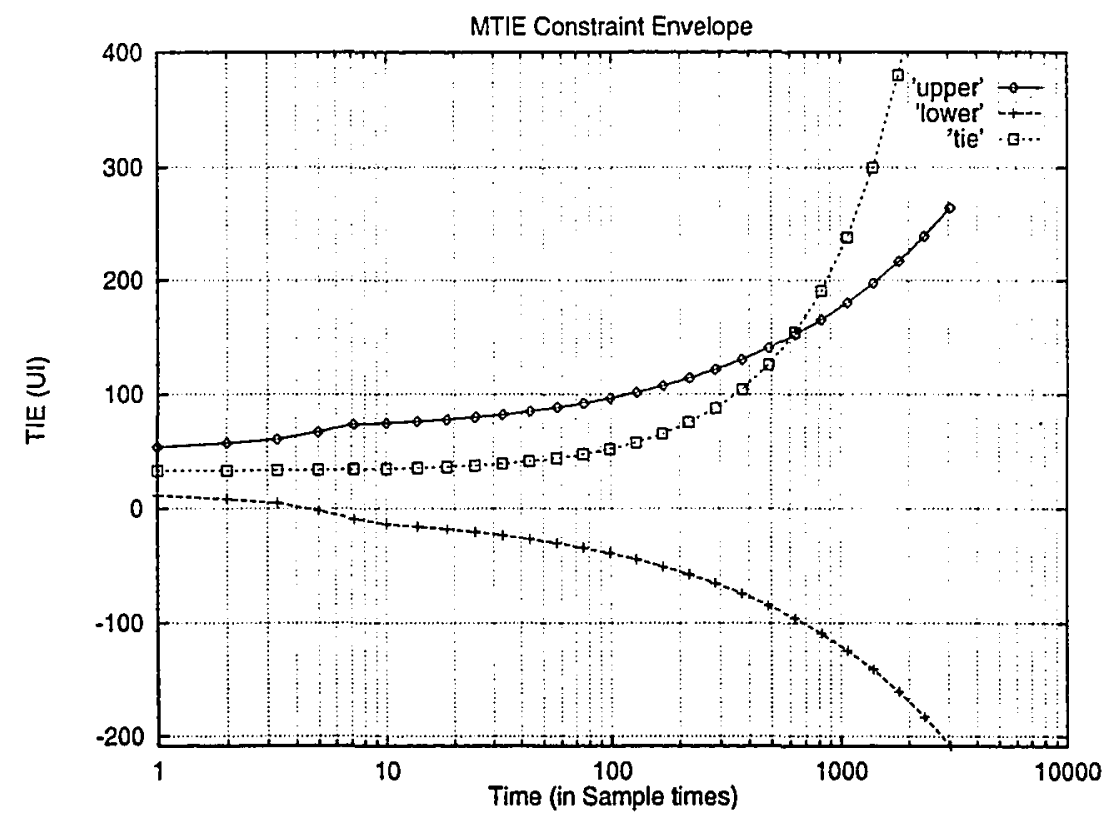

Figure 10. Actual MTIE Constraint Envelope with TIE Projection 


\section{CHAPTER IV}

\section{PREVIOUS WORK}

Published academic research in the area of Pointer Adjustment jitter and wander reduction has begun to appear only recently. An online literature search turns up less than 10 relevant journal papers and only one relevant masters thesis [11]. Quoting from a recently published paper [12]:

With the exception of [13] which focuses on oscillator design for digital Phase Locked Loops (PLLs), the present authors are unaware of any published paper devoted to this subject [analysis of pointer adjustment jitter and the search for efficient techniques to compensate for $i t$ ] which is of paramount importance to the operation of SDH networks.

However, Nunn [14] does give an analysis of the general requirements for jitter and wander reduction when transmitting PDH payload over SONET.

In the following sections we outline some of the major desynchronization techniques which have been studied. While it has been shown that some have the ability to attenuate jitter to levels set forth in the relevant standards, none specifically address the MTIE wander constraint or algorithms for satisfying it.

\section{A. PURELY ANALOG LOW PASS PLL}

It has been said [12] that an analog implementation of a SONET PJE jitter attenuation filter would be "virtually impossible" due to the necessity of an extremely low cutoff frequency. Instead, analog PLLs are augmented with pointer spreading 
techniques which ease the accuracy requirements of the analog components. Examples of such techniques are described below.

\section{B. PHASE SPREADING}

Figure 11 shows the basic architecture of the phase spreading technique [15].

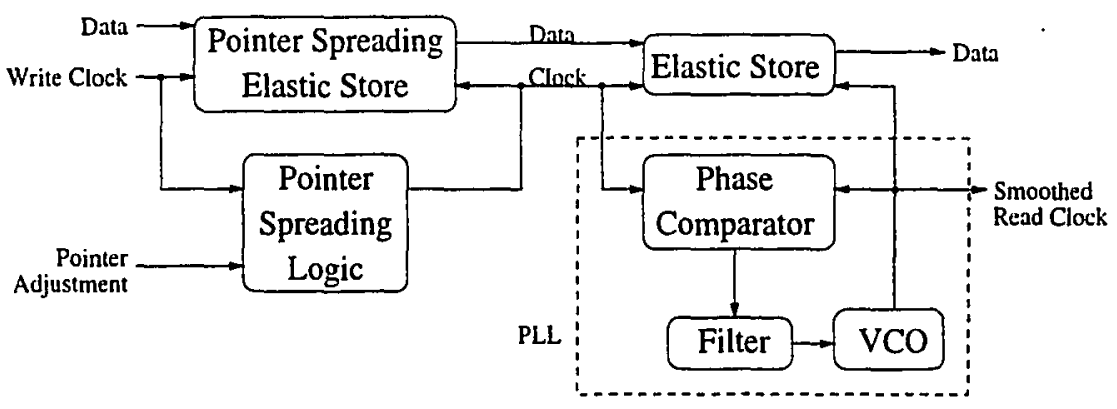

Figure 11. Architecture for the Phase Spreading technique

The main problem addressed here is the large magnitude of the phase transient resulting from a PJE. Rather than filtering the entire 8UI phase hit, a digital circuit captures the PJE and leaks it to the analog PLL one bit or fraction of a bit at a time. This strategy is sometimes also called "Bit Leaking". The pointer spreading logic in the above diagram is implemented in one of two ways: fixed rate and variable rate.

Kusyk [15] shows that with constant-rate pointer adjustments resulting from a $20 \mathrm{ppm}$ frequency offset, both fixed and variable rate pointer spreading techniques result in acceptable jitter magnitudes when applied to the DS1/VT1.5 mapping. Note, however, that SONET pointer processing allow sustained frequency offsets of up to $320 \mathrm{ppm}$ [16]. 


\section{FIXED RATE}

For the fixed rate method, some leak rate must be chosen beforehand. Slow rates result in superior jitter performance, but risk buffer overflow or underflow when excessive phase accumulates too rapidly in the Pointer Spreading Elastic Store. Larger leak rates reduce the risk of a buffer spill but generate additional jitter.

Still, under restricted operating conditions, this technique is capable of meeting the jitter constraints [15]. Unfortunately, fixed rate phase spreading is not guaranteed to meet jitter performance objectives for some payload mappings [12].

\section{VARIABLE RATE}

An improvement to the fixed rate method adjusts the leak rate based on the statistics of incoming pointer adjustments [17]. If a single PJE is encountered, it is leaked at a rate which generates negligible jitter. If a second pointer adjustment is encountered before the first is leaked to the PLL, the leak rate is increased to reduce the risk of a buffer spill. As might be expected, this modification allows variable-rate phase spreading to achieve better jitter performance than the fixed-rate method.

\section{E. STUFF THRESHOLD MODULATION (STM)}

Waiting time jitter poses a problem in desynchronizer design since its frequency components have no lower bound [6]. An approach was put forth by Grover et al. [18] wherein the threshold at which pointer adjustments occur is modulated at a relatively high frequency. The result is a stream of positive and negative PJEs which act to shift the jitter spectral components up in frequency to where they may be more readily attenuated by the low-pass filter of the desynchronizer [19].

This method was been studied theoretically by [15] and compared to pulsespreading techniques by [20], where it was shown to significantly reduce the jitter 
resulting from isolated PJEs. However, this strategy is implemented by the synchronizer and would require a change to the SONET standards. No move in this direction has been observed.

\section{F. ADAPTIVE DITHER BIT LEAKING}

This method, proposed by Sari and Karam [12], combines concepts from the two previous methods. Bit leaking is performed one bit at a time by a modulated, 128-bit pattern shown in Table I

\section{TABLE I}

Dither Pattern

$\begin{array}{llll}00000000 & 00010000 & 00100000 & 10000100 \\ 01000100 & 01001001 & 00101010 & 10101010 \\ 10101010 & 10101011 & 01101101 & 11011101 \\ 11011110 & 11111011 & 11110111 & 1111111\end{array}$

The modulated sequence is passed on to the low pass PLL where it resembles a ramp. The above pattern generates a zero to one transition but it may also be scaled in magnitude and duration to achieve the desired transfer of PJEs to the PLL.

In addition, an extension was suggested whereby a sequence of PJEs could be tracked by adjusting the slope of the the phase ramp such that the subsequent PJE would be canceled out just as it arrived.

\section{G. FEED FORWARD POINTER SPREADING}

This method requires that the synchronizer and desynchronizer work together. The synchronizer passes (in some manner) phase information to the desynchronizer so that when a pointer adjustment occurs, the desynchronizer will have anticipated the event and leaked the appropriate amount of phase already — resulting in a zero phase 
error upon arrival of the adjustment.

As will be seen in a later chapter, our controller is able to do precisely this when the PJEs occur in predictable cycles - without information from the synchronizer.

A system with this advanced information has the potential of jitter performance which is superior to other methods. However, both the sending and the receiving equipment must support this (non-standard) mode of operation. This technique does not operate with existing equipment and there is little evidence suggesting that the current trend will change.

\section{H. SUMMARY}

While some of the above techniques have been shown to attenuate jitter resulting from pointer adjustments, none is guaranteed to meet the constraints. Specifically, a violation of the buffer constraint results in data errors, and a violation of the MTIE constraint can lead to network instability and a loss of synchronization. This "selfevaluation" is the primary distinction between these techniques and the technique which was the subject of our investigation. We set out to determine if a controller could evaluate how a particular frequency change would affect the wander and buffer constraints before that frequency change was applied. We proposed that such a controller would be capable of satisfying both wander and buffer constraints for as long as possible. Jitter reduction was another goal, though not considered explicitly by the controller. 


\section{CHAPTER V}

\section{THE KNOWLEDGE-BASED CONTROLLER}

We set out to investigate a control methodology which could meet our two primary objectives. First, we wanted very small jitter compared with levels specified by ANSI for the standard PJE tests. This would represent good performance under normal conditions. Second, we wanted the controller to systematically satisfy the two primary constraints. Specifically, if the wander of the output approached the maximum allowed by MTIE specifications, we wanted the controller to adjust the output such that the wander constraint would not be violated until absolutely necessary — until the buffer was also in danger of overflow or underflow. Likewise, if the buffer were about to overflow, we wanted the controller to adjust the output in such a way that it wouldn't overflow until absolutely necessary. Finally, when all other options are exhausted, the controller would choose which constraint to violate, and maintain the other within specification.

Although we initially implemented a simple Proportional/Derivative-type controller, it soon became clear that more controller knowledge was necessary to accomplish this task. The prospect of incorporating expert knowledge in rule form seemed promising and we later found that a predictive knowledge-based controller was suitable to the task.

In Section A of this chapter we list some of the virtues of the heuristic approach to controller design and implementation. In Section B, the classical knowledge-based (or fuzzy-logic) controller is described. Finally, Section $\mathrm{C}$ presents the foundations of the PKBC applied to the desynchronization problem. 


\section{A. REASONS FOR CHOOSING AN HEURISTIC APPROACH}

\section{Goal Equations are Nonlinear}

First, it was our expectation that common-sense rules facilitate the design of a controller with acceptable performance in the usual case, thus satisfying condition one. While this reason permits an heuristic approach, it doesn't mandate one since other techniques can also meet jitter constraints.

MTIE and jitter calculations require peak-to-peak and other nonlinear operations - removing many linear controllers from consideration. A heuristic approach would specify appropriate actions as the wander and buffer values approached critical levels. In addition, if prediction were used, the result of any control action could be evaluated before that action is taken, adding confidence to its ability to operate within the constraints.

\section{Various Modes of Operation}

The goals of the PLL are slightly different when experiencing different modes of PJE generation. With rule-based approaches, such changes in operating regions are natural. Since the current mode (or its estimate) may be incorporated into each rule, smooth switching between operational strategies is possible.

\section{Opposing Constraints}

With no constraints on the buffer, ideal jitter may easily be realized. With no constraints on jitter generation, no buffer would be needed. It is the coupling between these opposing constraints which makes the problem such a challenge. A set of if-then rules seemed to be a natural way of expressing the desired actions of the controller when operating under varying conditions. 


\section{B. THE TRADITIONAL KNOWLEDGE-BASED CONTROLLER}

\section{What is it?}

a. Philosophy. The $\mathrm{KBC}$ is a type of expert system. The "expert" is typically a human who has the knowledge and experience necessary to control the system. The expert expresses his knowledge of the control task in the form of If-Then rules which use vague terms like "large" or "slow". The vague terms are defined by fuzzy sets [21] and an inferencing method is used to transform plant measurements into control actions.

b. Architecture. The $\mathrm{KBC}$ is an input/output system with a many-to-one mapping. The basic architecture consists of $\mathrm{N}$ inputs and a single output. If many independent outputs are desired, the basic architecture is replicated as needed. Each input variable is assigned some number of fuzzy sets, and each fuzzy set is assigned a label. Typically, the output is also specified in terms of a fuzzy set. The rules associate conditional statements with an output, and the truth of the conditional determines the strength of the association. The truth of a rule is sometimes called it's "firing strength".

c. Sets. Fuzzy set theory is a generalization of classical set theory. Whereas in classical set theory an element is either a member of a set or it is not a member of the set, fuzzy sets allow partial membership; the "membership function" of classical sets always evaluates to one (true) or zero (false) but the membership function of a fuzzy set can take on any value from zero to one.

Figure 12 illustrates these comparisons with both classical and fuzzy membership functions for the set of "tall" men. A man measuring 5' 10" would be classified as not "tall" by the classical membership function, but "tall" to degree 0.67 by the fuzzy membership function. The truth of a statement such as "John is tall" is synonymous with the degree of membership of the meaurement of John's height in the set "tall". 

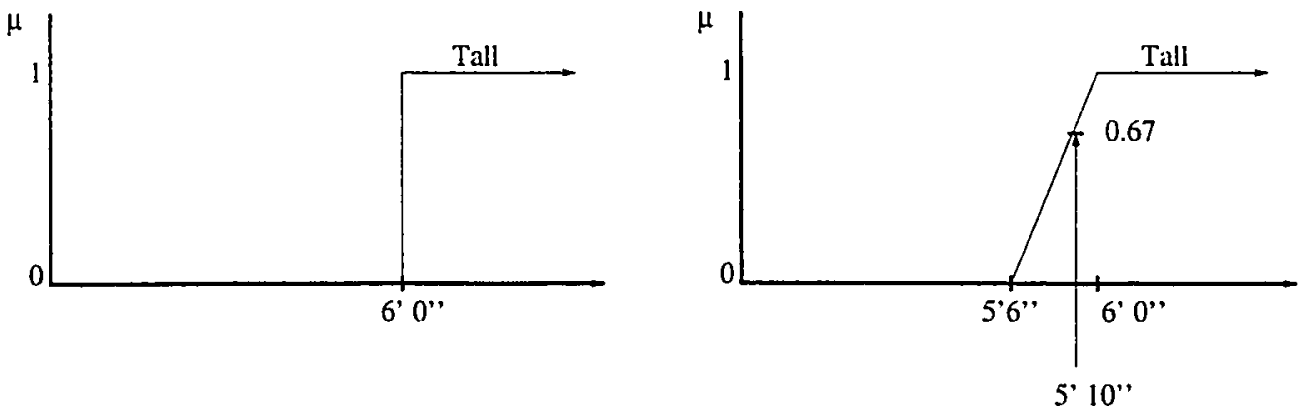

Figure 12. Crisp and Fuzzy Definition of "tall"

d. Logical Operators. Although there are many logical operators defined for fuzzy sets (see [22], for example), we present only conjunction (AND), disjunction $(O R)$, and complement (NOT).

- Conjunction/AND

The conjunction, or $A N D$ operator is typically defined by the the $\min ()$ function. For example,

$$
0.4 \cap 0.5=\min (0.4,0.5)=0.4
$$

An alternative definition is algebraic product. With this definition,

$$
0.4 \cap 0.5=0.4 \cdot 0.5=0.2
$$

- Disjunction/OR

The disjunction, or $O R$ operator is typically defined by the $\max ()$ operator. For example,

$$
0.4 \cup 0.5=\max (0.4,0.5)=0.5
$$

An alternative definition is $\min (a+b, 1)$. With this definition,

$$
0.4 \cup 0.5=\min (0.4+0.5,1)=0.9
$$


- ComplementNOT

The complement, or NOT operator is almost universally defined as $1-a$. For example,

$$
\neg(0.3)=1-0.3=0.7
$$

Notice that if $a$ and $b$ are constrained to the crisp set $\{0,1\}$, each logical fuzzy operator is reduced to the corresponding boolean definition.

e. Implication. Each input variable of a rule is fuzzified with the input fuzzy sets to produce a truth value. Then these truth values are combined with the logical operators to produce the aggregate truth of the conditional statement. This process is repeated for each of the rules, yielding a set of truth values and an action associated with each (the right hand side of each rule). Some method must be used to determine how each desired action should be modified in the case of a conditional statement with a truth value less than one.

The most common inferencing method was proposed first by Mamdani [23] and states that the output fuzzy set of a rule $r$ is

$$
Y_{r}(x)=\min \left(K_{r}, C_{r}(x)\right)
$$

where $K_{r}$ is the truth of the antecedent and $C_{r}(x)$ is the stated consequence of the rule (the "then" part of the rule). If the output fuzzy sets are triangles, this inference scheme produces trapezoids - triangles with flattened tops.

Another method is product inference. This method states that the output fuzzy set of a rule $r$ is equal to the product of the truth of the antecedent $K_{r}$ and the stated consequence $C_{r}$

$$
Y_{r}(x)=K_{r} \cdot C_{r}(x)
$$

With this method, the general shape of the output set is not changed but is simply scaled 
proportionally.

f. Defuzzification. Once all the rules have been evaluated, the resulting output fuzzy sets (modified according to inferencing method) must be combined to produce a single output value. Typical methods include: the centroid, the maximum value, the median value, the weighted sum of the maximums, height defuzzification, and others [24]. The center of sums is the most common defuzzification method and consists of computing the weighted average of each resulting output fuzzy set.

g. Controller Example. We demonstrate the above with a simple desynchronizer controller. We begin by defining the controller's inputs and output. The inputs consist of the buffer level, and its derivative. The output, or control effort, is a frequency adjustment to the DDS, in Hz. The next step is to define fuzzy sets on the input and output domains. Figure 13 shows a possible configuration.
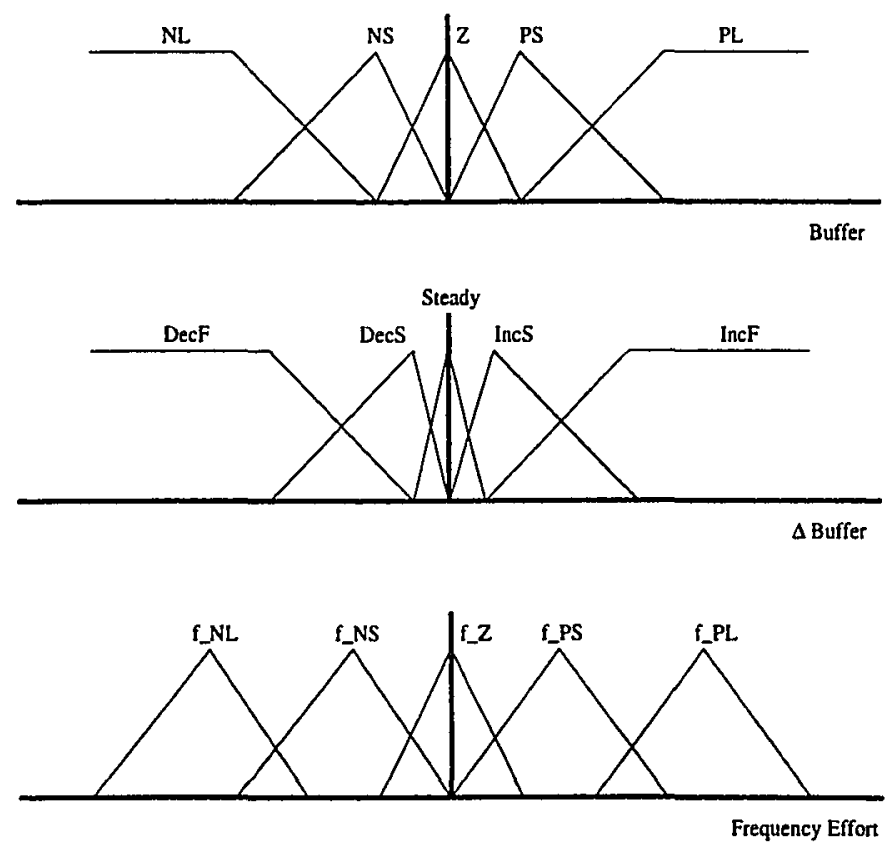

Figure 13. Fuzzy Set Definitions for Sample KBC 
where NL, NS, Z, PS, and PL stand for "Negative Large", "Negative Small", “Zero", "Positive Small", and "Positive large" respectively. For the $\Delta$ Buffer input, Dec and Inc stand for "Decreasing" and "Increasing" respectively, and the trailing F and S stand for "Fast" and "Slowly" respectively. The preceding $f_{-}$in the third plot indicates that these fuzzy sets correspond to the "frequency" output and to distinguish them from the sets of the Buffer input variable. Notice that for the input fuzzy sets, adjacent fuzzy sets overlap 50\%. It has been shown in [25] that this and other considerations lead to faster rise times and less overshoot [24]. Next, rules which map an input state to an output set are constructed. Rules have the form

IF buffer IS [ ] AND $\triangle$ buffer IS [ ] THEN effort IS [ ] and may be represented by a two-dimensional matrix as seen in Figure 14.

\begin{tabular}{|l|l|l|l|l|l|}
\hline \multicolumn{5}{|c|}{ Frequency } \\
\hline \hline \multirow{2|}{*}{ ferror $^{\text {error }}$} & NL & NS & Z & PS & PL \\
\hline \hline IncF & f_Z & f_NS & f_NS & f_NS & f_NL \\
\hline IncS & f_PS & f_Z & f_Z & f_NS & f_NS \\
\hline Steady & f_PS & f_Z & f_Z & f_Z & f_NS \\
\hline DecS & f_PS & f_PS & f_Z & f_Z & f_NS \\
\hline DecF & f_PL & f_PL & f_PS & f_PS & f_Z \\
\hline
\end{tabular}

\section{Figure 14. Rule Matrix}

The rule matrix states that if the buffer is PL and the rate of change is IncS, then the desired action is to decrease the output frequency by the amount specified by f_NS. The task of constructing the rule matrix requires knowledge of the control task and is often assisted by simulation.

Due to overlapping of the fuzzy sets, there will usually be two active rows and two active columns for any input values. This implies that as many as four rules will fire - to different degrees - resulting in four scaled or clipped output fuzzy sets. 
These output fuzzy sets are then defuzzified using one of the methods mentioned above to produce a single output value.

Since the KBC is a stateless input-output system, it is possible to characterize its transfer function by applying all possible input pairs and plotting the resulting surface, such as that shown in Figure 15 where we see that the control surface of this $\mathrm{KBC}$ is nonlinear.

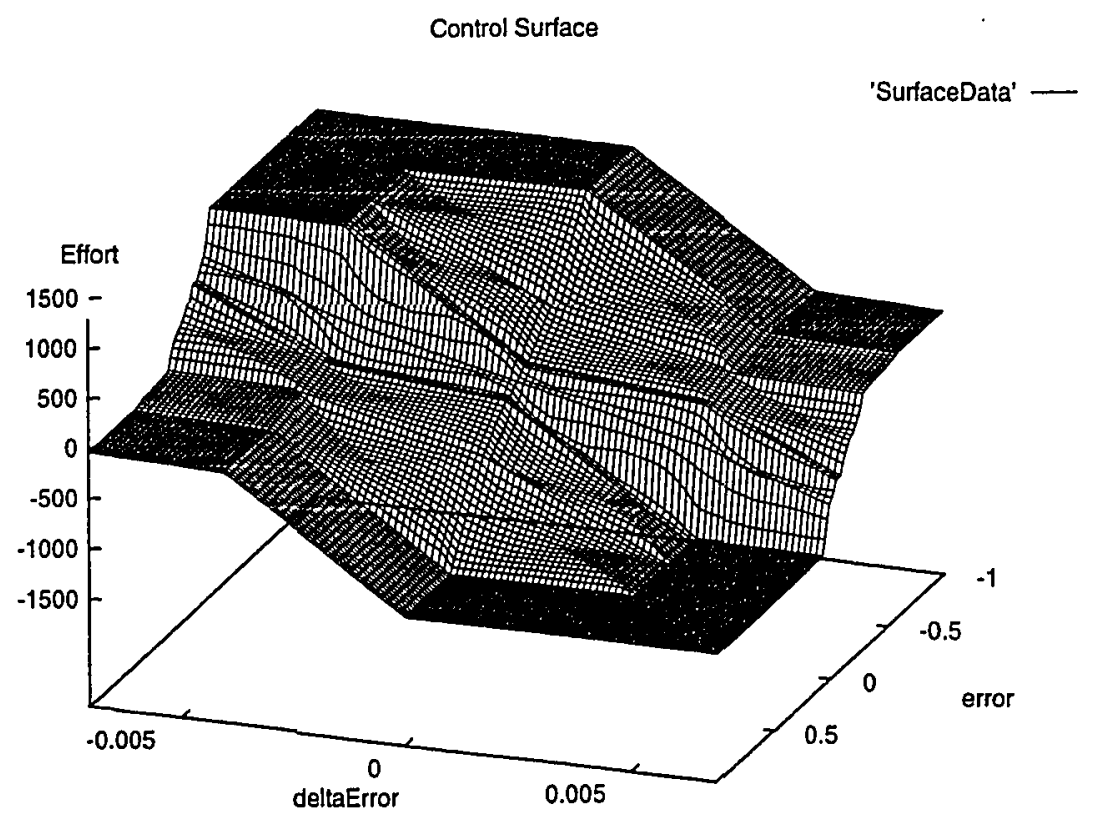

Figure 15. KBC Control Surface

Output saturation is due to the flatness of the input fuzzy sets at extremes. [26] describes the conditions under which linearity may be achieved. 
2. When is a Fuzzy Knowledge-Based Controller appropriate?

a. Difficult or Intractable Mathematics. There are control applications where it is very difficult to characterize the physics of a system due to its complexity, nonlinearity, or changing parameters. The design of a fuzzy controller doesn't require a mathematical plant model, but instead relies on an implicit understanding of the plant's operation and knowledge of which actions are appropriate under which circumstances.

b. Accelerated Update Rates. Fuzzy sets may be represented discretely with only a few points, and can be easily stored in a small memory [27]. Also, the common operations on fuzzy sets are easily implemented in hardware, and often do not require multiplication or division. Thus an entire controller may be easily implemented by an inexpensive microcontroller. Tools exist which map a fuzzy controller into lookup tables and inferencing routines. The parallel nature of the algorithm also makes it a candidate for concurrent processing.

In addition, specialized stand-alone VLSI chips are capable of evaluating as many as 30 million rules per second and are available for only a few dollars [28].

c. Non-Mathematical control objective. If the control objective is not readily expressed mathematically, analytical design methods tend to break down. In other situations, the control objectives may be expressed mathematically, but are more easily expressed as if-then rules.

d. Universal stability proof not required. Many critics will claim that there exists no mechanism for ensuring the stability of a fuzzy controller. While stability analysis such as is available for linear controllers does not apply to fuzzy controllers, there are nonlinear stability analysis techniques which may be used to demonstrate, for example, asymptotic stability. Still, these techniques are rarely used in practice, and the stability of most applications of fuzzy logic is verified by simulation or extensive tests. See [24] 
for a survey of stability methods.

\section{Disadvantages}

a. No algorithm/mathematical algorithm for design/tuning. The rulebase of any knowledge-based system will only be as good as the expert who constructed the rules. Of course the expertise of both designer and controller increase as performance is observed and appropriate changes to the controller are made, but the process often follows a path of trial-and-error.

b. Stability. As mentioned earlier, stability studies have not yet been conducted for the PKBC paradigm. Applications where such guaranteed characteristics are mandatory must look to other control strategies.

\section{PREDICTIVE KNOWLEDGE BASED CONTROLLER}

\section{What is it?}

a. Philosophy. Predictive Knowledge-Based Control (PKBC) is an extension of standard $\mathrm{KBC}$. Like $\mathrm{KBC}$, it uses fuzzy sets and fuzzy set operators to evaluate the truth of each rule statement. However, unlike standard PKC, the PKBC uses prediction to evaluate the effect the stated action would have on the state of the system at a future time. The predicted state is then evaluated in the same way as the current state was evaluated by the KBC.

If the input and plant processes are well represented by the predictive models, rules which would result in an undesired state are eliminated from consideration. This can result in improved functionality and reliability. 
b. History. PKBC was developed in Japan in the early 1980s by a group led by Yasunobu [29], [30], and [29]. Their goal was to replace human subway operators with automatic, computational controllers. After interviewing operators (the experts) they identified several relevant performance parameters:

- Passenger safety

- Energy consumption

- Adherence to schedule

- Passenger comfort

- Speed limit constraints

- Accuracy of stopping at stations

A person driving a vehicle builds an internal model of the vehicle's mechanics which is consulted to decide if, for example, the current deceleration rate will result in the desired stopping position or if some adjustment is necessary. This concept was used to extend the standard $\mathrm{KBC}$ to a Predictive KBC. The group found that the above performance parameters could be met through the application of a set of carefully crafted rules of the form:

if ( $u$ is $A \rightarrow x$ is $J$ and $y$ is $K$ and $z$ is $L$ )

then $u$ is $C$ sub $i$

For example,

If (when the power control is on coast, the safety is good and comfort is good and energy consumption is good) then coast

A model of the train's dynamics is used to predict the state that would result from the action $u=A$ and other models predict the value of the variables $X, Y$, and $Z$. Finally, the truth of the rule is evaluated using the fuzzy sets $J, K$, and $L$, and the candidate action associated with the rule with the highest truth value is chosen and applied to the output of the controller. 
This control method makes concurrent use of three techniques: fuzzy set theory, which evaluates multiple system objectives; artificial intelligence in the form of experiential If/Then rules; and real-time simulation and prediction of control results [31].

Simulations comparing PKBC to PID control were encouraging. Compared to a PID controller, the standard deviation of stopping error was $10 \mathrm{~cm}$ for $\mathrm{PKBC}$, and $28 \mathrm{~cm}$ for the PID. For riding comfort, the PKBC made 6.6 command changes versus 16.0 by the PID (which equates to a smoother ride). Field tests of PKBC on the subway in Sendai Japan produced results similar to the simulations. The PKBC exhibited more expert, human-like characteristics than other controllers, and conserved energy compared to other methods (including human control). The controller was still in active operation in 1995.

As a side note; even under automatic control there is still a human operator near the train's controls as a backup. Comparisons between human control and PKBC are made with a human operator who - to retain his skills - operates the train during nonpeak hours.

Other applications of PKBC include car parking [32] and the control of mobile robots [33]. In both cases, the controller uses prediction to evaluate a set of candidate actions and choose the one deemed most appropriate.

c. Architecture. The PKBC adds models and a predictor to the standard $\mathrm{KBC}$ architecture as illustrated in Figure 16.

Each rule in the rulebase is evaluated in turn. The candidate action of the rule is passed through the predictor which generates "virtual measurements" - measurements which would be expected in the future if the proposed action were taken. The measurements are fuzzified and the inferencing rule determines the strength of the rule. The optimal action is then chosen based on the firing strengths of the rules. 


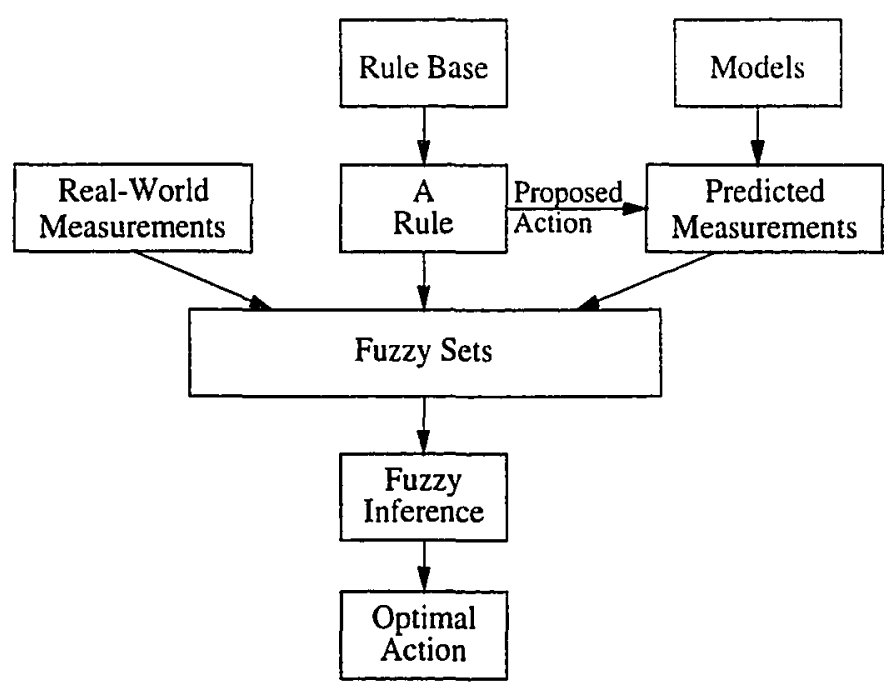

Figure 16. PKBC Execution Diagram

d. Rules. We developed a new rule structure for our controller. The Yasunobu method, described above, consisted of an action and the results desired from that action. We modified the grammar to allow an independent conditional statement before prediction. This may also be seen as combining standard (conditional-only) rules with the standard predictive rules. We also removed the redundant action from the end. Figure 17 illustrates our new grammar.

The conditional part of the rule is the same as before, but now a candidate action is included in each rule. An additional step uses prediction models to estimate the truth of the dependent condition based on the proposed action. The rules may include any number of terms, and any number of rules are allowed.

Our inclusion of the conditional statement is a departure from the architecture originally developed by Yasunobu. If the prediction models are complete and accurate, the two architectures are largely the same. In particular, if the conditional statement is removed from each rule, the two architectures are precisely the same. Our modification 


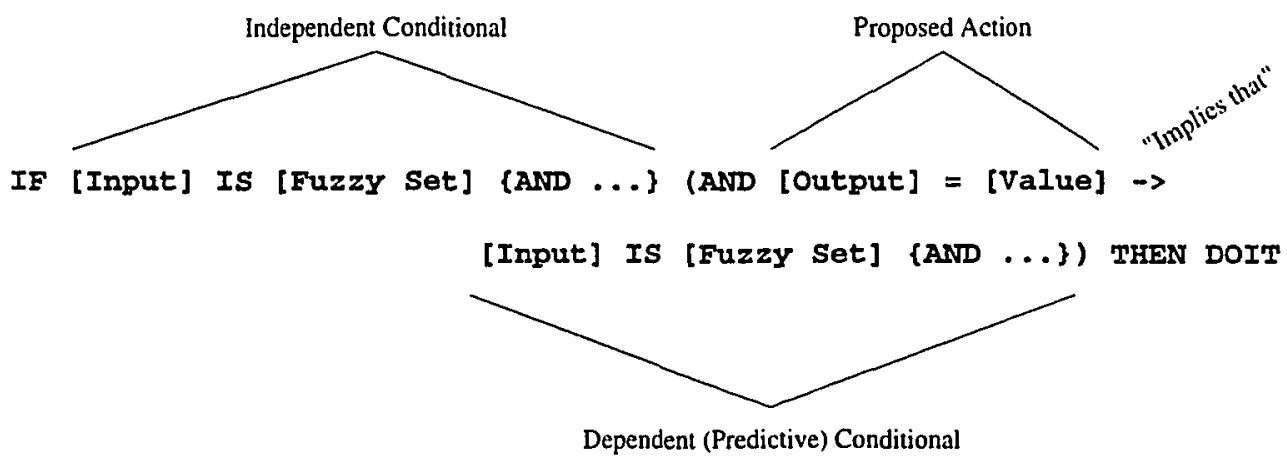

\section{Figure 17. New PKBC Rule Structure}

allows the use of actual physical measurements in the evaluation of rules, while the standard architecture only evaluates predictions. The importance of this difference increases as the accuracy of the predictive models decreases, since the controller may use physical measurements in addition to predicted results to help it decide which action is most appropriate.

e. Advantages. The use of prediction is like adding an additional feedback loop in the control system. A candidate action is passed through a set of plant models and the results evaluated to ascertain the goodness of that action. If sufficient candidates are available, something close to the best action will be chosen and applied. This selfevaluative approach yields several advantages over simpler feedback control methods.

One advantage is the ability of the controller to operate within hard constraints. In a desynchronizer, for example, the buffer must not overflow. Each rule may reflect this constraint by including a predictive term such as "and buffer is OK".

PKBC also has the potential of requiring less tuning than a standard $\mathrm{KBC}$. The knowledge of a $\mathrm{KBC}$ is completely contained in the content of the rules and the definition of the fuzzy sets. Rules are defined in terms of existing conditions and desired actions. Choosing a desired action requires that the designer have or obtain a good 
understanding of the effects that action will have. With $\mathrm{PKBC}$, the designer still must have some knowledge of appropriate actions in different conditions, but if several candidate actions are given in otherwise identical rules, the controller is able to choose the one which results in the best performance. Using language of Kosko [27], the odds of a "sabotage" rule firing are low, since the predicted results would be poor.

Also, when several constraints cannot be satisfied simultaneously, the designer may specify which one to violate by adding rules which ignore the lesser constraint when the more important one is near violation.

Finally, our addition of a conditional statement to the rule structure allows greater flexibility and potentially greater tolerance to model inaccuracies, as described in Section ld above.

\section{When is a PKBC Appropriate}

If there is no model available for the system, classical control techniques are severely disadvantaged and $\mathrm{KBC}$ or adaptive technique [34] may be able to provide a solution. If there is a linear model available for the plant and the controller objectives are expressed similarly, then one of many control techniques may be employed, with proven results. However, if a model is available (which may be arbitrarily complex and nonlinear) and the controller objectives are difficult to express symbolically, then PKBC may be a viable option.

\section{Disadvantages}

The author is not aware of any study of the stability of the PKBC. This is an important concern for some applications and, although our simulations have demonstrated stable behavior for many varied scenarios, conditions for global or asymptotic stability have not yet been derived. 
Also, offering many candidate actions can lead to a large number of rules, which increases computation time. This is also true as the number of inputs increases since there are many more combinations of the input state. However, although we specify nearly 60 rules, the number could be reduced to below 15 if the grammar were changed to allow a rule to specify a set of candidate actions rather than just one. (This change would certainly affect the complexity of the rulebase, but would not be expected to affect the time necessary to evaluate the rules).

Another disadvantage of PKBC over other methods is the need for simulation in the design and evaluation of the controller. This disadvantage is not unique to PKBC, however.

Despite these disadvantages, simulation has suggested that the control system is stable, the computational load is within the reach of modern processors, and a reasonably limited number of inputs leads to an acceptable number of rules. 


\section{CHAPTER VI}

\section{PKBC FOR THE SONET/DS3 DESYNCHRONIZER}

\section{A. OVERVIEW}

A SONET/DS3 desynchronizer has one operational objective: Smooth the clock signal without causing a buffer underflow or overflow. This objective may be decomposed into three concrete goals:

- Keep the buffer half full to minimize the probability of either underflow or overflow,

- Keep the output frequency near enough to the nominal DS3 value that the wander specification (MTIE) is not violated, and

- Exhibit low-pass behavior so that jitter generation is minimized.

The first two goals have explicit limits: the buffer has a fixed size, and MTIE is bounded by the MTIE mask specified by Tl committee documents. This means that a properly designed controller could conceivably evaluate itself in terms of these consirainis and take actions predicted to yield acceptable results.

It is not possible, however, to meet the third goal explicitly, because there is no universally applicable jitter specification. Instead, jitter levels are specified in terms of specific PJE profiles. Thus, assuming that the controller does not identify and adapt to specific PJE tests, the desynchronizer must be designed, tested, and tuned using trialand-error.

We determined that a PKBC-based approach is both possible and appropriate for 
the desynchronization problem. It is possible because prediction models of the buffer level and MTIE compliance may be derived. We decided that PKBC is appropriate for this constraint satisfaction problem because of its ability to predict the result of an action before it is taken and our presumed ability to construct an appropriate rulebase.

In the remaining sections of this chapter we describe the inputs, models, and output of the PKBC-based desynchronizer controller followed by insights and strategies for rule and fuzzy set development.

\section{B. ARCHITECTURE}

The PKBC-based desynchronizer is illustrated in Figure 18

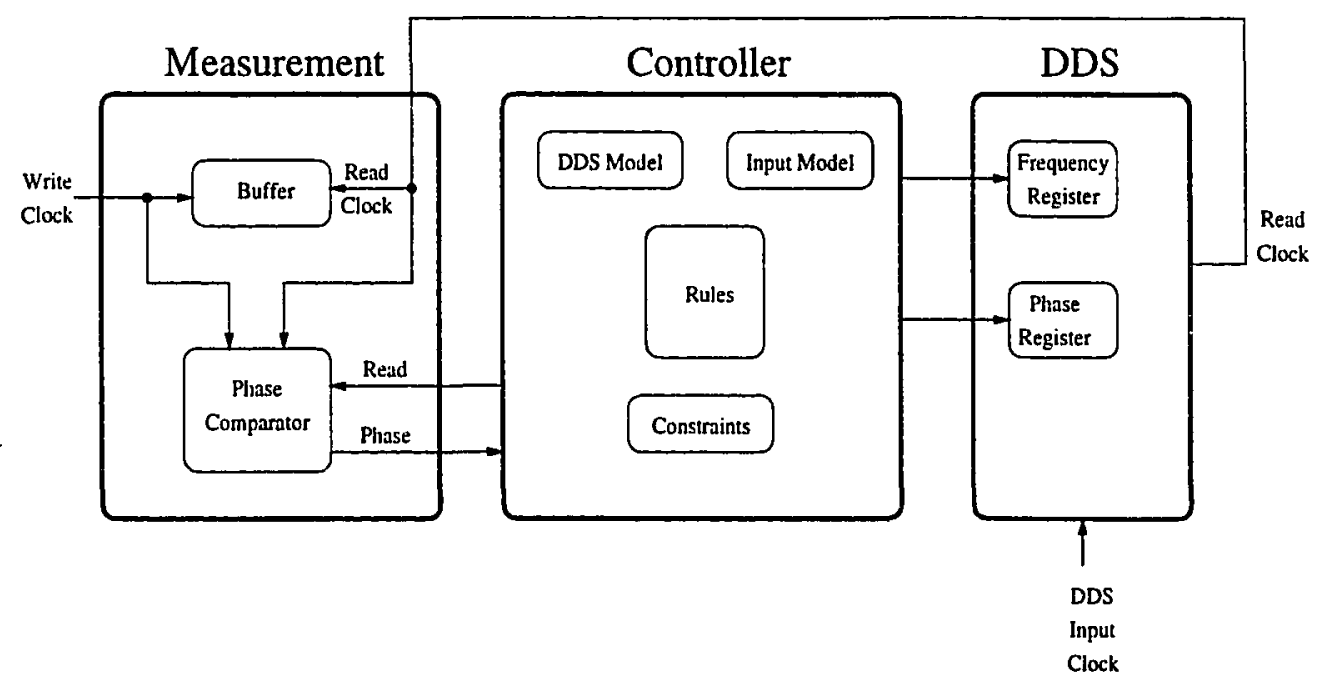

Figure 18. Basic Desynchronizer Diagram

The "measurement" block sends phase measurements to the controller. The "controller" is a computational device which executes the PKBC algorithm and sends frequency (and/or phase) commands to the DDS. The DDS generates the output clock which is also fed back to the measurement block. 
An expanded view of the "controller" may be seen in Figure 19.

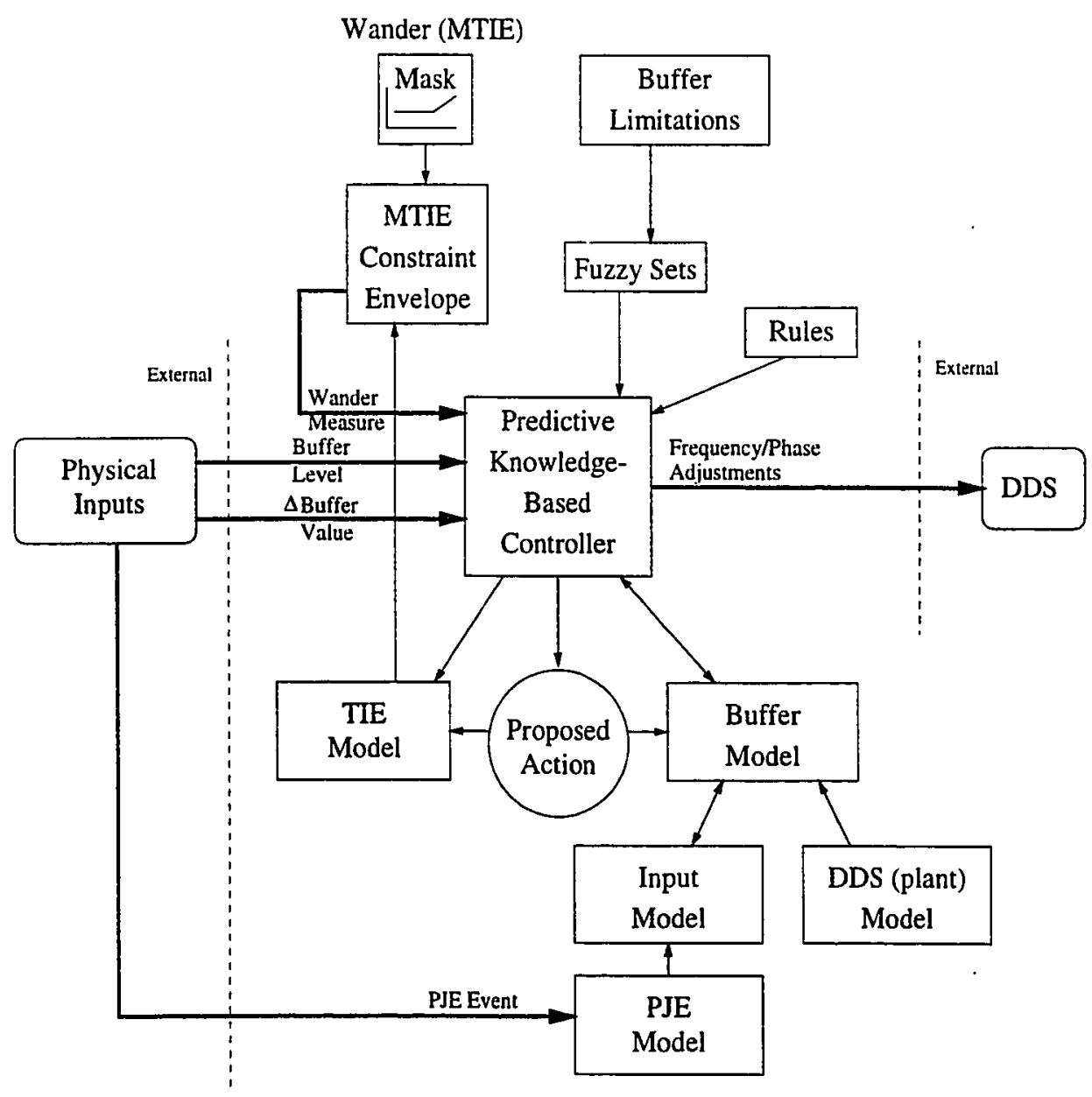

Figure 19. PKBC Desynchronizer Controller

The main inferencing block entitled "Predictive Knowledge-Based Control" in Figure 19 is described in Section $\mathrm{C}$ of Chapter V and thus will not be explained again here. The leftmost and rightmost blocks are the input and output interfaces to the external world respectively. The purpose of the controller is to send commands to the output (the DDS) such that the measurements received from the inputs (including the internally-generated "wander level" input) will meet the operational goals of the system. 
The remaining blocks above and below the PKBC are supportive functions and models which are described in more detail in the following sections.

\section{Inputs}

a. Buffer Level. The buffer, or phase measurement is synonymous with the filllevel of the elastic store, which may also be characterized by

$$
\text { buffer }=\int_{0}^{t} f_{\text {out }}-f_{\text {in }} d t
$$

where $f_{\text {out }}$ is the output frequency and $f_{\text {in }}$ is the incoming frequency. The buffer level is measured at the end of each SPE row (explained in Section A.2 of Chapter II). and accumulated until the sample time of the controller is reached.

The level of the buffer is measured at the end of each STS-1 row, resulting in a rate of $8000 \frac{\text { frames }}{\mathrm{sec}} \cdot 9 \frac{\text { rows }}{\text { frame }} \cdot 1 \frac{\text { sample }}{\text { row }}=72,000 \frac{\text { samples }}{\text { second }}$. Measurements taken more often would be plagued by "jitter" caused by the fixed stuffing bits and overhead gaps. Measurements taken more slowly would lack the resolution that may be achieved by oversampling and averaging.

At the end of the row, either 621 or 622 bits of DS3 payload data - depending on whether or not the stuff opportunity was taken - will have been written into the buffer. Ideally, the same number of bits will have been read out of the buffer by the output clock in order that the buffer doesn't fill or empty. We will call these SPE-row-based phase samples sub-samples to avoid confusion with the overall control-system sample period $T_{s}$.

The buffer measure may be thought of as a counter with its up-count input connected to the write clock and its down-count input connected to the read clock. If we measured the buffer once per control algorithm sample time, a resolution of 1UI would 
be the limit (without more capable phase measurement equipment, such as [35]).

The sub-samples are averaged to produce the buffer measurement used by the controller;

$$
\frac{\sum(\text { BufferLevel }[i]-\text { BufferSize/2) }}{i}
$$

where BufferLevel[ $i]$ is the buffer sample after each row. Assuming a controller sample period of 0.04 seconds, approximately 2880 row measurements are accumulated before Equation (3) is evaluated.

Oversampling of the SPE-row results in a maximum resolution of

$$
\text { Resolution }_{\text {buffer }}=\frac{1}{\text { row frequency } \cdot T_{s}}
$$

For a sample period of $T_{s}=0.04 \mathrm{~s}$,

$$
\begin{aligned}
& =\frac{1}{8 \mathrm{KHz} \cdot 9 \cdot 0.04 \mathrm{~s}} \\
& =3.47 \times 10^{-4} \mathrm{UI}
\end{aligned}
$$

This theoretical limit on the phase resolution may not be realized if the stuffing ratio is the quotient of small integers (like $1 / 3$ or 6/10). This is evidenced in Section A.l of Chapter 8 where mapping jitter is plotted as a function of the stuffing ratio. Fortunately, sustained integer stuffing ratios would not be expected in a real network where random processes are at work.

b. $\Delta B$ uffer Value. The derivative of the buffer level is estimated by a first order backward difference, normalized to the sample period;

$$
\Delta \text { buffer }[i]=\frac{\text { buffer }[i]-\text { buffer }[i-1]}{T_{s}}
$$

Large or sudden changes in $\Delta$ buffer - following a PJE, for example - limit the 
usefulness of this measure. The predicted $\Delta$ buffer (described in a Section B.4.a) is more representative, since the effect of periodic PJEs are considered over a long period of time.

c. Wander Measure. Whereas the buffer measure is an absolute measure (the number of bits from half full), wander is defined in terms of the amount of time before the MTIE mask would be violated if the current frequency were maintained. This measure is also called "time-till-violation". It is derived by first generating the MTIE Constraint Envelope and superimposing it with the TIE samples which would result from the proposed or current frequency action. Then a search is performed to determine where TIE crosses the envelope. For example, a wander value of 30 means that the current or proposed frequency will violate MTIE in 30 sample times. If the tie line does not cross the envelope, a very large number is returned.

If a candidate action is predicted to result in an MTIE time-till-violation of less than one sample period, the action is obviously not acceptable, since MTIE would be violated before a subsequent control action could be taken to avert it.

d. Comments. Both buffer and wander may be measured either in terms of timetill-violation or the distance from their current position to the constraint ceiling.

However, we chose to represent the buffer input by the absolute fill-level rather than the predicted time-till-violation, due to the transient nature of the buffer measure. If an unexpected PJE event occurs, for example, the previously predicted time-tillviolation is meaningless. However, such sudden and unexpected changes are not seen in the wander measure since wander is a function of only the output clock - over which the algorithm has complete control. Thus, the time-till-violation measure is more predictable and longer-term estimations are justified. 


\section{Rules}

Our implementation of PKBC rules have the following form:

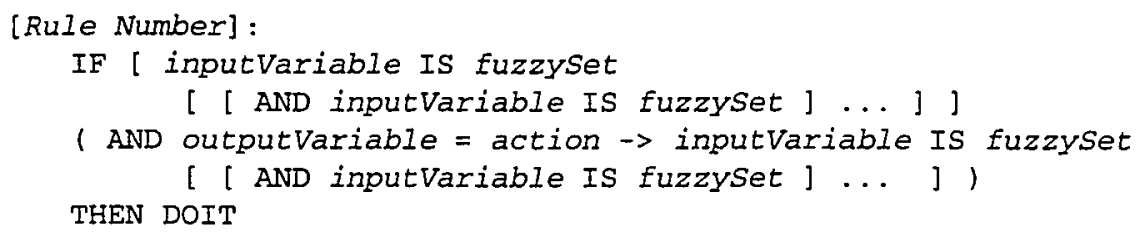

Each rule begins with a rule number, used for easy reference, followed by the reserved word IF and the conditional terms. Then, in parenthesis, an output action is proposed and the predicted result is evaluated in by the predictive terms.

The rules are stored (one per line) in a text file and parsed by a custom parser using lex [36] and yacc [37]. The parser enforces adherence to the grammar and ensures that every referenced input (like buffer) and fuzzy set (like NL) is defined elsewhere. The result is a doubly linked list of rules and terms at runtime. The rulebase may contain an arbitrary number of rules, each with an arbitrary number of terms. Modifications or extensions to the grammar are easily accomplished by simply editing the appropriate lex/yacc description files and rebuilding the executable.

\section{Fuzzy Sets}

Each fuzzy set used in the PKBC is read and parsed from an input file at runtime.

The file is divided into sections, each identified with one of two keywords: inputvar or outputvar followed by the variable name like buffer, d_buffer, or wander. (We will refer to the derivative of buffer as $\Delta$ buffer in textual discussions and d_buffer in relation to the simulator). After the declaration of the variable name, a terms statement declares the number of fuzzy sets defined for that variable. Then the appropriate number of fuzzy sets are defined.

Each fuzzy set definition consists of a name and one or more real numbers 
separated by spaces or tabs. Our representation of fuzzy sets allows four profiles. The singleton is used to define candidate outputs and is defined by a single number. The triangular membership function [38] is the most commonly used type and is defined by three numbers (left corner, peak, and right corner). A trapezoid is defined by four numbers (left corner, left peak, right peak, right corner), and if the first two or last two numbers are equal, a ramp is assumed. A ramp differs from a trapezoid in that one end of the ramp never returns to zero, and thus its domain extends to infinity. Ramps are useful for specifying default behavior outside standard input domains.

Figure 20 and Table II illustrate our representation of fuzzy sets.

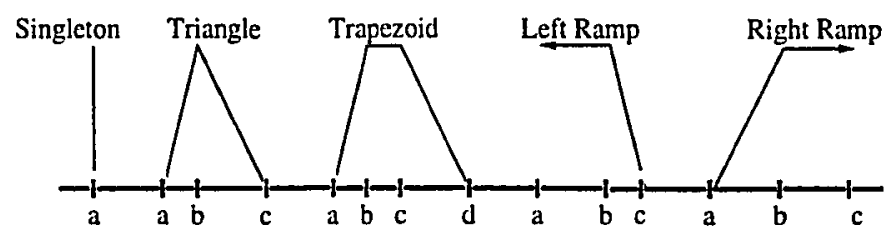

Figure 20. Fuzzy Set Definitions

TABLE II

Fuzzy Set Definitions

\begin{tabular}{|l|c|c|c|c|}
\hline $\begin{array}{c}\text { Fuzzy Set } \\
\text { Type }\end{array}$ & \multicolumn{4}{|c|}{$\begin{array}{c}\text { Numerical } \\
\text { Definition }\end{array}$} \\
\hline \hline Singleton & a & & & \\
\hline Triangle & a & b & c & \\
\hline Trapezoid & a & b & c & d \\
\hline Left Ramp & a & a & b & c \\
\hline Right Ramp & a & b & c & c \\
\hline \hline
\end{tabular}

The buffer constraint is embodied within the fuzzy sets, as described in Section C.1 below. 
4. Models

Two types of models are used in the simulator: Simulation Models and Prediction Models. To simulate the operation of the control algorithm, certain real-world processes must be emulated. The frequency of the original DS3, the frequency of the SONET network, the mapping of the DS3 payload into an STS-1 SPE, and the generation of PJEs must all be modeled by the simulator. Since the simulation models are not part of the PKBC controller they are described in the next chapter on simulation.

A predictive controller must possess and maintain a model of each real-world process necessary for the evaluation of candidate actions. In a simulation environment, all relevant information is contained in the simulation models mentioned above. But in a real-world implementation, the future DS3 rate, for example, is not known exactly by the controller and must be estimated. The Input Model, for example, characterizes the observed DS3 signal and is used to estimate future DS3 rates such that the "truth" of the predictive-term of each rule may be evaluated.

Prediction models are updated as new measurements are taken and only utilize data which would be available to the controller in a real-world implementation. Following is a description of the prediction models used by the controller.

A. Buffer Model. As stated earlier, the theoretical buffer level is determined by

$$
\text { buffer }[i+1]=\text { buffer }[i]+\int_{i}^{t+T_{s}} f_{\text {out }}-f_{\text {in }} d t
$$

Assuming that the $f_{\text {in }}$ and $f_{\text {cut }}$ remain constant between samples, this may be rewritten as

$$
\text { buffer }[i+1]=\text { buffer }[i]+\left(f_{\text {out }}-f_{\text {in }}\right) T_{s}
$$

Both $f_{\text {oul }}$, the output frequency of the DDS and $T_{s}$, the sample period of the control system are known quantities. The model for $f_{\text {in }}$ is described in the next section. 
The predicted $\Delta$ buffer value is computed by calculating the difference between the current buffer measurement and the predicted next buffer measurement.

B. Input Model. The goal of the Input Model is to estimate the frequency of the original DS3 signal. To accomplish this, it assumes that notice of PJE events is available and also receives phase measurements. The assumed availability of PJE information is reasonable since other techniques, including the phase-spreading techniques [15], utilize this information.

From Equation (4) we can derive an expression for $f_{\text {in }}$

$$
f_{i n}=\frac{\text { buffer }[i-1]-\text { buffer }[i]}{T_{s}}+f_{0}
$$

While this might be an accurate model, the estimate of $f_{\text {in }}$ changes drastically whenever a PJE occurs due to the sudden change of eight to 24UI. Thus we represent $f_{\text {in }}$ with two separate models; one to estimate the input frequency without the effect of PJEs, and another model to estimate the rate at which PJEs occur. Ultimately, the two models are combined to form a more representative estimate of the input frequency. In fact, the resulting estimate remains as constant as the instantaneous $f_{\text {in }}$ frequency and the PJE rate.

Thus, when a PJE occurs, the PJE model is passed the polarity and timestamp of the event, and its effect is removed from the instantaneous phase measurement. However, this second action requires additional explanation.

Consider the example where a phase sample is taken at $25.0 \mathrm{~ms}$ which yields a difference of 10UI. Next, a negative PJE occurs at $25.75 \mathrm{~ms}$, and the next sample is taken at $26 \mathrm{~ms}$. Although the correct buffer level at $26 \mathrm{~ms}$ is $18 \mathrm{UI}$, the measurement returns something less - for the following reason. Since the first $75 \%$ of the sub-samples measure 10UI and the last $25 \%$ measure 18UI, an average measure of $12 \mathrm{UI}$ is returned. (The subsequent phase sample at $27 \mathrm{~ms}$ correctly yields $18 \mathrm{UI}$ ). This effect is modeled 
by including the term $P J E_{1}^{s t}[i]$, which indicates the amount of phase seen at time $i$ from a PJE during the previous sample time (0.75.8 in the example above) and $P J E_{2}^{n d}[i-1]$ which contains the remainder ( $0.25 \cdot 8$ in the example above).

Thus input frequency at sample $i$ is estimated by the following expression:

$$
\frac{p[i-1]-p[i]+f_{D D S}[i-2] T_{d}+f_{D D S}[i-1]\left(T_{s}-T_{d}\right)+P_{D D S}[i-1]-P J E_{I^{s}}[i]-P J E_{2^{n ! l}}[i]}{T_{s}}
$$

where $p[i]$ is the PJE-removed measurement at sample $i ; f_{D D S}[i]$ is the DDS frequency applied $T_{d}$ seconds after sample $i$; and $P_{D D S}[i]$ is the change to the DDS phase register (in UI). $T_{d}$ is used to simulate the time between the instant when the sample is taken and the time when the algorithm finishes calculating the desired DDS action. When asked for the average input frequency, the Input Model combines the estimate above with the estimated PJE bit rate (described in the next section).

By decomposing a phase measurement into its two components - changes due to a frequency offset and steps due to PJEs - this model can provide a very accurate estimate of past input frequency values. Our use of past estimates for future prediction is validated by the fact that instantaneous input frequencies and PJE statistics change slowly over time [3]. In fact, as will be seen in Chapter 8, the controller actually anticipates the occurrence of regularly occurring PJEs and compensates for them before they arrive.

The input model requires input parameters specifying the length of the averaging filter and, if low-pass filtering of the average input measurement is desired, the filter's cutoff frequency.

C. PJE Model. The PJE model predicts future PJE events. Since PJE statistics are likely to change slowly [2], past events are a good indication of future events. Thus, a list of recent PJEs are recorded, along with their polarity and the time at which they occurred. From this list, an average PJE rate may be calculated. 
If, in our implementation of the PJE model, a stream of PJEs occur at regular intervals and suddenly an expected PJE doesn't arrive, the estimated PJE rate is ramped down to zero such that a rate of zero is achieved at the time of the $\mathrm{n}^{\text {th }}$ expected PJE.

A more representative PJE model might have multiple levels of estimation. The first level would acquire the instantaneous PJE rate. Missing PJEs from the first level would be modeled by a second level and spurious or bursts of PJEs by a third. Although this approach would yield a more accurate model of the PJE profiles used in the tests (and greatly reduced jitter), we decided that a simpler, more general PJE model which was not tailored to the standard tests would be more appropriate for real-world operation.

The input parameters associated with this model include the number of past PJEs to remember, and $n$.

D. TIE Model/MTIE Constraint Envelope. The MTIE Constraint Envelope module transforms an MTIE mask and past and future TIE samples into the constraint envelope described in Chapter III. This module is also able to calculate the time at which the TIE — resulting from a proposed frequency - crosses the envelope, indicating an MTIE violation.

The envelope is sent TIE samples at a fixed rate. Each of these samples is passed through a $10 \mathrm{~Hz}$, low-pass filter, but they are only stored in the TIE collection at the rate corresponding to the minimum observation time of the MTIE mask, since smaller observation times are not constrained by MTIE.

The envelope is updated each time a TIE value is stored. Then, for each rule, a candidate action is passed to the envelope, and a time-till-violation measurement is requested. A predicted TIE plot is compared to the envelope, the time of the first crossing is determined, and interpolation is employed to increase the resolution of the returned measurement. 
The TIE model is (by definition) described by Equation (2) which may be found in Section C of Chapter II. For simplicity we do not low-pass filter the calculated future TIE samples while searching for the crossing. This has the effect of advancing the point of crossing since a low-passed ramp is delayed with respect to an unfiltered ramp. Thus, the simplification results in a slightly more conservative estimate.

E. Direct Digital Synthesis (DDS) Model. The output of the control algorithm is a frequency command which is sent to the DDS for realization. In this section we will develop a model for the frequency generated by the DDS. Figure 21 illustrates the primary components of a DDS.

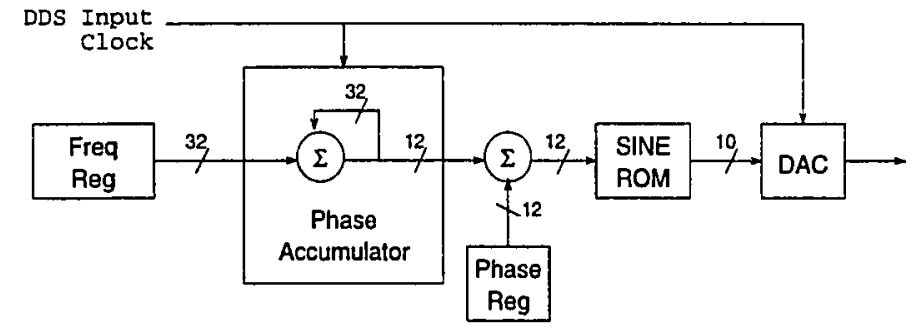

Figure 2.1. DDS Block Diagram

The frequency register holds the Frequency Tuning Word $\left(T W_{F}\right)$ and the phase register holds the Phase Tuning Word $\left(T W_{P}\right)$. During operation, $T W_{F}$ is accumulated once for every cycle of the DDS input clock and the result is added to $T W_{P}$ before being used to address the sinewave lookup table ROM. The addressed value of the ROM is passed through a Digital to Analog Converter (DAC) to form the output clock of the control system.

For an example, consider a $T W_{F}$ of one. The address used in the ROM lookup is incremented after each DDS input clock cycle, resulting in an output frequency equal to the DDS input clock rate $\left(f_{\text {clock }}\right)$ divided by the length of the ROM $\left(2^{R O M_{\text {his }}}\right)$. If $T W_{F}$ holds one third of the length of the ROM, the frequency of the output equals the input 
clock rate divided by three.

The frequency of the DDS is modeled by the following equation

$$
f_{D D S}=\left(\frac{T W_{F}}{2^{\text {ROM }}}\right) f_{\text {clrsck } k}
$$

where $R O M_{B I T S}$ is the number of bits in the ROM address.

The input parameters to the DDS model include the frequency of the input clock, the length of the tuning word, and the initial frequency and phase.

\section{Output}

The output of the PKBC is the candidate action which was predicted to result in best performance. Although the DDS is capable of adjusting both the frequency and phase of the output, our rules specify only frequency adjustments.

\section{STRATEGIES FOR RULE/FUZZY SET DEVELOPMENT}

In the process of designing rules and fuzzy sets, many different configurations were discovered. Although not every attempted set of rules and fuzzy sets yielded a satisfactory controller, we were pleased with the overall robustness of the algorithm and its tolerance of significant changes in operating point, input disturbances, and modifications to the rules and fuzzy sets. Surprisingly, a rather small set of rules is sufficient to provide acceptable performance.

The rules are divided into two primary classes. The first class of rules apply under "normal" conditions - when neither MTIE nor buffer constraints are near violation. A second set of rules define the constraint-satisfaction mode and are active when one or more of the constraints approach their limit. The two classes of rules are structurally identical and are distinguished only by the regions over which their fuzzy sets are defined. For example, the rule 
IF buffer IS PM AND d_buffer IS SI AND

wander IS OK AND ( freq = NS $\rightarrow$

wander IS OK AND buffer IS PM ) THEN DOIT

belongs to the first class, since it applies only when the proposed action is predicted to leave buffer and jitter within bounds.

By first ignoring the explicit MTIE and buffer constraints, we proceeded to develop a controller which resulted in "good" jitter performance. Sections 1 through 3 below apply to this mode of operation. Section 4 describes the candidate actions and Section 5 describes how the "normal" rules are augmented to ensure MTIE compliance at the cost of spilling the buffer when necessary. Section 6 describes how the rules are augmented to ensure buffer compliance. Finally, Section 7 provides miscellaneous insights and observations for rule development.

\section{Partitioning the Space}

Large frequency steps generate jitter. Thus when small frequency offsets are possible, jitter performance improves. If the buffer level is "OK", then no adjustment is necessary. If the buffer level is moderately large, a moderately large frequency adjustment is warranted. When the buffer is about to overflow, a very large effort is allowed - within the constraints of wander generation.

To accomplish this, we first partitioned the buffer space into regions: Zero Positive $(Z P)$ and Zero Negative ( $Z N)$, Positive Small (PS), Positive_Large ( $P L)$, and Positive_Very_Large $(P V L)$. Negative values were named similarly. These regions correspond to our goal of achieving good jitter performance in the usual case. A fifth region, Positive Too Large (P2L) activates rules whose objective is constraint satisfaction, corresponding to our goal of satisfying the constraints when necessary. We consider the constraint-satisfaction regions in Sections five and six but for now we will assume that the wander and the buffer values are well within limits. 


\section{Delta Ruts}

Rather than use the buffer measure as the setpoint of the controller, which could result in large frequency offsets, overshoot, or ringing, we decided to control the rate at which the buffer changed. Figure 22 shows the actions of the controller in two dimensions.

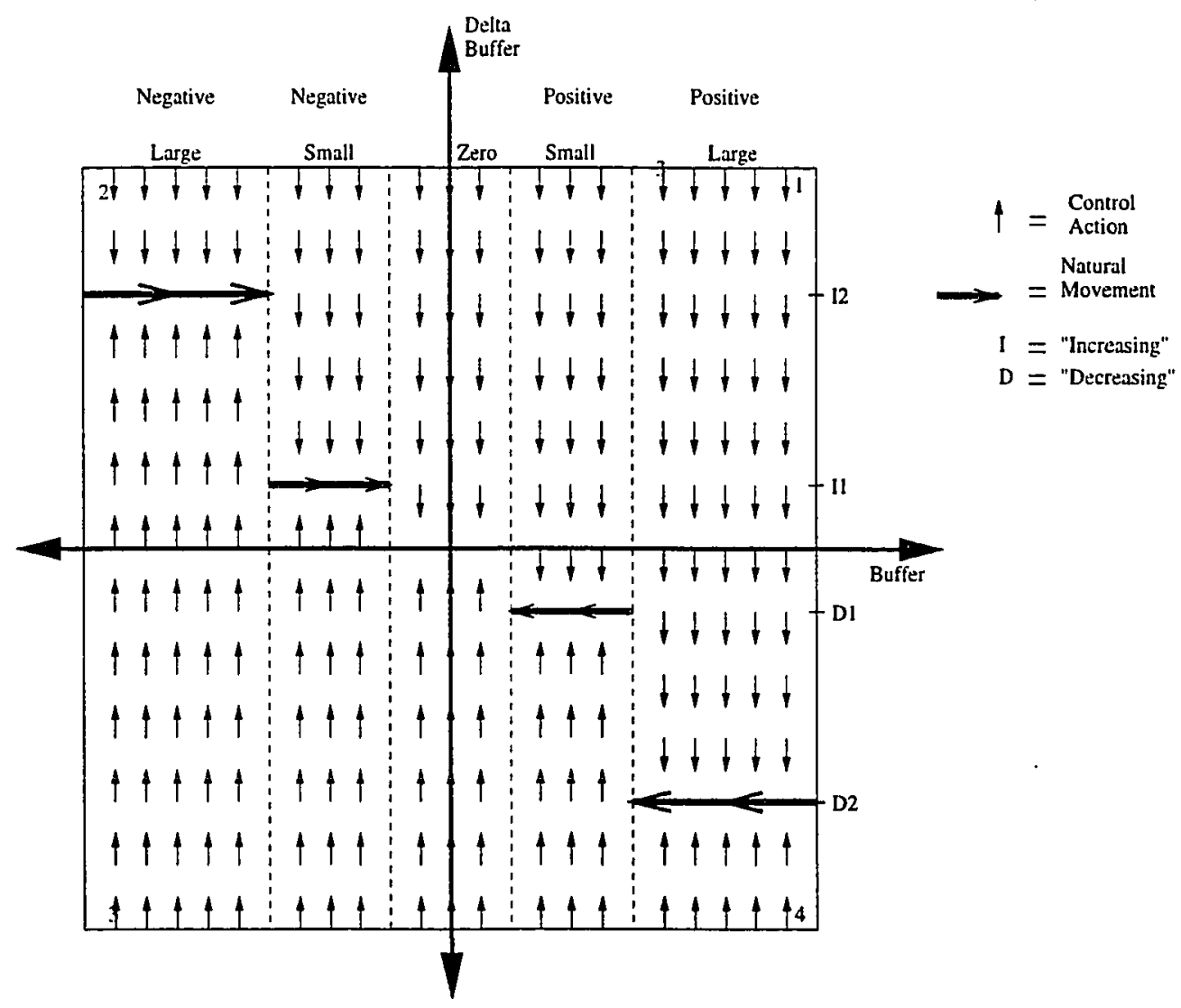

Figure 22. Natural and Controlled Vector Fields

The $x$-axis represents the current buffer level. The $y$-axis represents the first derivative of the buffer level, or $\Delta$ buffer. In this discussion we will represent the "state" by the pair (buffer, $\Delta$ buffer). The vertical arrows represent the actions of the controller. The horizontal arrows denote natural state motion. For example, if the derivative of buffer is 
negative, the state of the system will tend toward the left, indicated by the left-pointing arrow.

If the state is in the first quadrant, the buffer is more than half full and is becoming more full. This is undesirable for obvious reasons and a negative $\Delta$ buffer is desired. If in the second quadrant, the buffer is less than half full iut is filling. If the state is in the third quadrant, the buffer is less than half full and is continuing to become less full. The fourth quadrant represents the state where the buffer is more than half full, but is emptying. Clearly quadrants two and four contain states which act to prevent buffer overfiow.

Next, we partition the x-axis. For simplicity, we define only five partitions: Zero, Positive Small, Positive Large, Negative Small, and Negative Large as shown. We decide that if the buffer is positive and Small we want it to Decrease at some rate D1, chosen by some means. D2, I1 (rate of Increase), and I2 are chosen in the same way.

\section{Full Domain Fuzzy Set Spanning Property}

We chose to make predictions based on a time of one sample period. This implies that the predictive terms of the rule must be achievable in a single sample period or a low firing strength will result. We found that the best way to deal with this is to cause all the $\Delta$ buffer fuzzy sets to span the entire domain. Thus, since each predicted consequent is always true to some degree, the action which moves the state of the controller closest to the peak of the fuzzy set (optimizes the function) is chosen.

Consider, for example, the situation where buffer is NS (Negative Small), and the desired d_buffer value is $S I$ (Slowly Increasing). If we define $S I$ as shown in Figure 23, the predictive part will evaluate to zero if none of the listed candidate actions is predicted to place d_buffer precisely within the domain of IS. If the predictive part evaluates to zero, the truth of the entire rule will be zero and the rule will not fire. The 
probable result is a situation where no rules fire.

To solve this problem, we simply require all "target" fuzzy sets to span the entire range, as shown in Figure 23. By "target" fuzzy sets we mean a fuzzy set which is part of the predicted outcome of a rule.

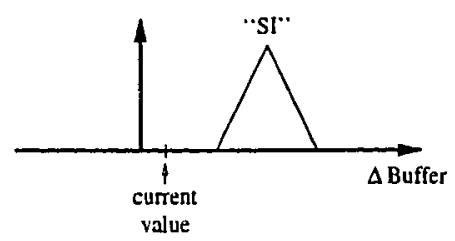

Figure 23. Original D_Buffer Fuzzy Set Definition

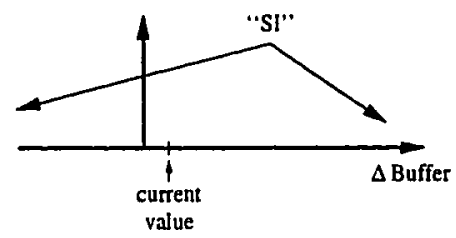

Figure 24. Improved (for PKBC) D_Buffer Fuzzy Set Definition

With this spanning definition, the statement $d_{\text {_buffer }}$ is $S I$ is always true to some degree. If a candidate action causes an increase in the degree of truth for this term its overall truth value will increase. With everything else equal, the rule which maximizes the predicted parameters will have the largest truth value and will be chosen. (Notice that this assumes a definition of conjunction based on the product operator. See Section 7 for the justification of this choice.)

In short, if a fuzzy set is the goal of one or more rules (it occurs in a predictive term), it should span the entire region over which it might be applied. If a fuzzy set is not the goal of any rule (it occurs in only conditional terms), it may be defined over whatever region is appropriate. 


\section{Output Fuzzy Sets}

Candidate actions for our PKBC are singletons or scalars. Figure 25 illustrates the output values we used in our simulations. $P$ implies Positive, $N$ implies Negative, VS stands for Very Small, $S$ stands for Small, $L$ stands for Large, and VL stands for Very Large. Units are $\mathrm{Hz} / \mathrm{sec}$.

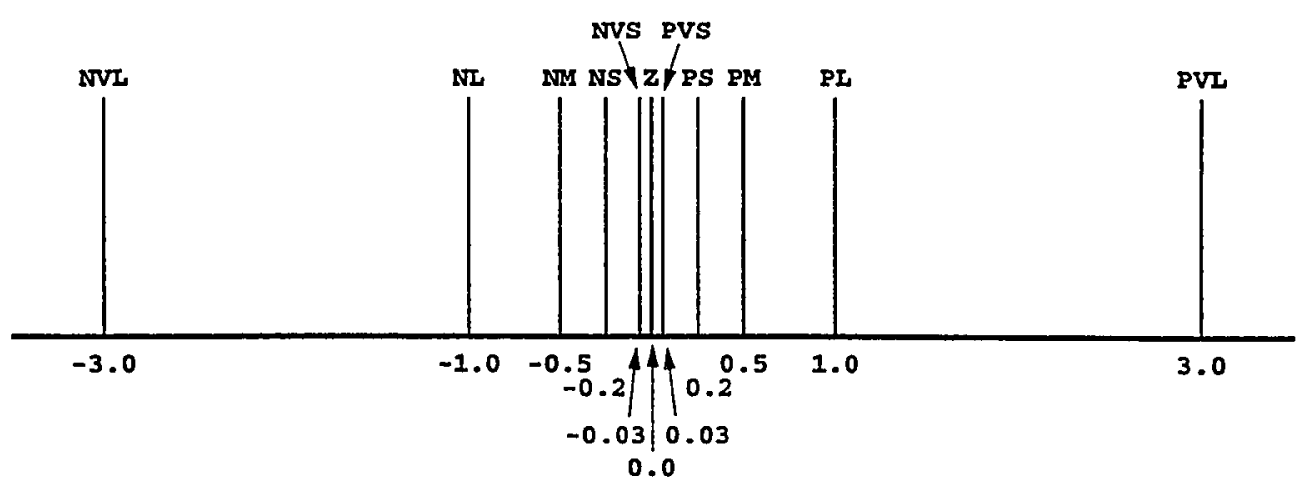

\section{Figure 25. Output Singletons}

The maximum rate at which the output frequency may change is determined by the largest defined output frequency step. If the largest proposed action were $\Delta f_{\max } \mathrm{Hz}$, the largest sustained rate of change in the input frequency would also be $\Delta f_{\max } \mathrm{Hz}$ per second.

The maximum frequency offset is typically applied only when the buffer is about to overflow, and maximum effort is warranted. Smaller buffer levels may be reduced to zero with smaller effort - thus only small candidate actions are specified in the appropriate rules in an effort to reduce jitter generation.

\section{Maintaining MTIE Compliance}

Once rules were written to maintain a buffer level of zero (half-full), adding MTIE compliance was relatively simple. First, the term 
.... AND wander IS POK

was added to all the rules. This ensured that actions which would result in a violation or near-violation of MTIE would evaluate to zero and be eliminated from consideration. Remember that a wander value of 21 , for example, means that the proposed action is predicted to violate MTIE in 21 sample times.

Next, a set of rules was crafted which stated that if wander were not OK and a candidate action would cause it to be more $O K$ than it was, apply that candidate action. Finally, if MTIE were violated, and a candidate action would result in moving TIE closer to the envelope (closer to re-compliance), then apply that action. The net effect of these rules is to cause the buffer to be kept at zero unless MTIE is in danger of violation. In that case, the action necessary to keep the violation from occurring is taken. These results are illustrated in Chapter 8.

\section{Maintaining Buffer Compliance}

We also show, in Chapter 8, that it is possible to construct the rulebase such that the buffer is given precedence over MTIE when both are near violation. Again, this was a relatively simple task. First, the term

$$
\text { ... AND buffer IS } O K
$$

was appended to every rule in the ruleset which ensured MTIE compliance. Then, two "ramp" fuzzy sets were defined on the buffer axis: Positive Too Large (P2L) and Negative Too Large (N2L), and the two fuzzy sets POS and NEG were defined on the d_buffer axis at +25 and -25 respectively. Finally, rules were added to the rulebase which stated:

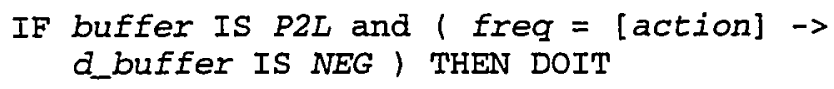

A similar rule was added for $N 2 L$. 
These rules ensure that if the buffer becomes too full or empty, appropriate actions will be taken to ensure that they do not increase (or decrease) further without regard for MTIE compliance. The location of $P 2 L$ and N2L define the boundary at which these buffer-constraints take effect.

Notice, however, that this action alone does not guarantee buffer compliance. If, for example, the "too large" fuzzy sets were located 20 bits from the actual buffer limits, a single 3-pointer burst would cause the buffer to spill before the controller would have time to react. Thus, buffer compliance may be guaranteed only if appropriate limits are placed on the rate and magnitude of PJE bursts, and the rate at which a continuous PJE stream changes.

\section{Observations}

Under most circumstances, a single term need not be in both the conditional and predictive terms. The question to ask is: Is this term a prerequisite for this action, or is this term something that I want to come about due to this action. In addition, if a single term is included twice, the product AND operator uses its truth value twice, decreasing the overall truth of the rule. Conversely, this property might be useful in a situation where a squared fuzzy set is desired.

Our implementation of the PKBC can employ either min or product definitions of the AND and inference operators. We can also specify whether the centroid or "winner-take-all" defuzzification method is employed. However, we have found that the product definition is superior to min and that centroid defuzzification does not make intuitive sense.

For example, suppose we have two rules as follows:

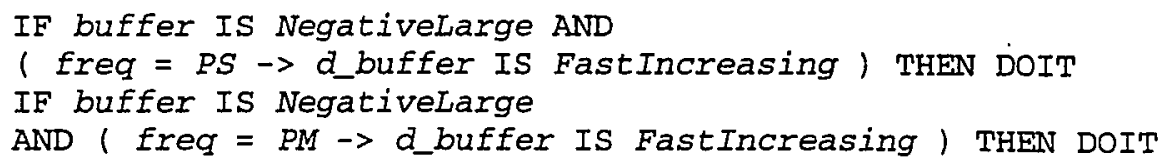


Now suppose that the truth of the conditional term is 0.2 and the truth of the predictive terms of the first and second rules are 0.6 and 0.9 respectively. With the min definition of AND, both rules will have the same overall truth value $(\min (0.2,0.6)=\min (0.2,0.9)=0.2$,$) yet clearly the second rule proposes the most$ appropriate action since it produces better compliance with the term IncreasingFast. The product definition of AND is thus more appropriate for a predictive controller as we have defined it, yielding rule truths of 0.12 and 0.18 respectively.

In addition, we believe that centroid defuzzification is counter-intuitive. The performance of each rule is evaluated using measurements and prediction. If we then weight each action based on the truth of the rule from which it came and combine them all into a single action, it is not clear that the result will have a better predicted outcome than the rule with the highest truth value. In fact, if a large action produces the most benefits, combining it with other actions with smaller benefits will reduce the overall effectiveness of the controller. Thus, we use a winner-take-all technique for rule selection.

A possible extension would be to combine the most promising candidate actions to form a new rule which would then be evaluated and compared to the rules from which it was derived. As before, the rule with the largest truth value would be chosen.

\section{FINAL CONTROLLER SPECIFICATION}

We now describe the fuzzy sets and rules which were used in the simulations of the next chapter to test jitter performance. Since neither constraint was threatened in these tests, the controller employed the rules which act to keep the buffer half-full. Although the constraint-satisfaction abilities were not demonstrated in the jitter tests, the rules were written such that MTIE was the dominant constraint. 


\section{FUZZY SETS}

We chose to partition the buffer level into five regions for positive (partly full) levels and five regions for negative (partly empty) levels. The fuzzy sets Zero Positive $(Z P)$, Positive Small (PS), Positive Large ( $P L)$, Positive Very Large $(P V L)$, and Positive Too Large $(P 2 L)$ - along with their negative counterparts - are illustrated in Figure 26.

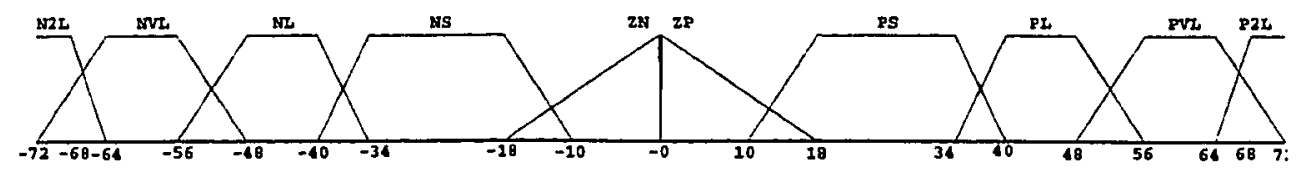

\section{Figure 26. Final Buffer Fuzzy Set Definitions}

Since the fuzzy sets of $\Delta$ buffer are the goal of one or more rules, they span the entire useful range of the variable. If a $\Delta$ buffer larger than MI is equally acceptable, the set may be defined by a ramp rather than a triangle.

The use of IS (Increasing Slowly) caused a conflict with the reserved word IS of the rule grammar, so we changed the order of the terms, yielding, for positive values: Slowly Increasing (SI), Moderately Increasing (MI) and Fast Increasing (FI). Both positive and negative values around zero are specified with the fuzzy set $O K$. These fuzzy set definitions are illustrated in Figure 27

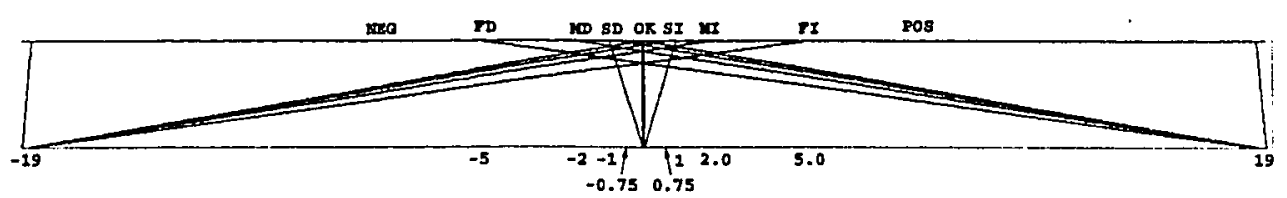

Figure 27. Final $\Delta B u f f e r$ Fuzzy Set Definitions

Notice that the only parameter which must be chosen for $\Delta$ buffer is the peak of each 
triangular fuzzy set. The endpoints of each set are assigned a value which is larger than the largest expected $\Delta$ buffer measurement.

MTIE is evaluated in terms of the elapsed time before a candidate action would result in an MTIE violation as indicated by the MTIE constraint envelope. Thus, large wander values are good and small values are bad. The wander value returned is negative if TIE falls outside the envelope. Figure 28 illustrates the fuzzy sets associated with wander.

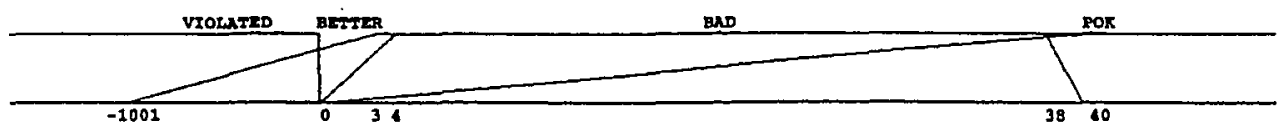

\section{Figure 28. Wander Fuzzy Set Definitions}

Although we also have the ability to measure $\Delta$ wander, we did not find its use necessary.

\section{RULES}

If two or more rules share the same truth value, whichever is first in the rulebase is chosen. Thus the rulebase begins with rules which propose frequency changes of zero since unnecessary changes in frequency produce unnecessary jitter.

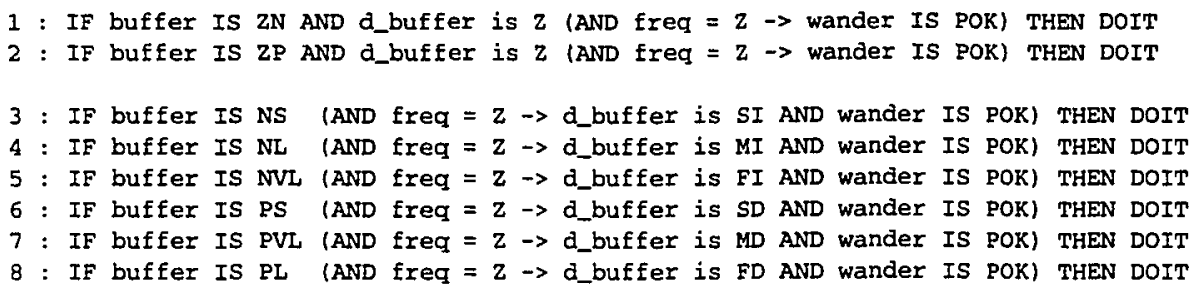

The second cluster of rules deal with normal operation - when neither MTIE nor buffer constraints are in danger of violation. Their goal is to maintain an average buffer level of zero. Notice that none of the rules in this section are applied unless they result 
in acceptable wander. Also, when the buffer level is near zero, only very small actions are permitted.

9 : IF buffer IS $2 \mathrm{~N}$ (AND freq = PVS $\rightarrow$ d_buffer is $\mathrm{z}$ AND wander IS POK) THEN DOIT

10: IF buffer IS $\mathrm{ZN}$ (AND fxeq $=$ PS $\rightarrow$ d_buffer is $z$ AND wander IS POK) THEN DOIT

11: IF buffer IS ZP (AND freq $=$ NVS $\rightarrow$ d_buffer is $z$ AND wander IS POK) THEN DOIT 12: IF buffer IS $Z P$ (AND freq $=N S \rightarrow$ d_buffer is $Z$ AND wander IS POK) THEN DOIT

13: IF buffer IS NS (AND freq $=$ PS $\rightarrow$ d_buffer is SI AND wander IS POK) THEN DOIT 14: IF buffer IS NS (AND freq = NS $\rightarrow$ d_buffer is SI AND wander IS POK) THEN DOIT 15: IF buffer IS NS (AND freq $=\mathrm{NM} \rightarrow$ d_buffer is SI AND wander IS POK) THEN DOIT 16: IF buffer IS NS (AND freq $=$ PM $\rightarrow$ d_buffer is SI AND wander IS POK) THEN DOIT

17: IF buffer IS NL (AND freq $=$ PS $\rightarrow$ d_buffer is MI AND wander IS POK) THEN DOIT 18: IF buffer IS NL (AND freq $=$ NS $\rightarrow$ d_buffer is MI AND wander IS POK) THEN DOIT 19: IF buffer IS NL (AND freq $=$ PM $\rightarrow$ d_buffer is MI AND wander IS POK) THEN DOIT

20: IF buffer IS NL (AND freq $=N M \rightarrow$ d_buffer is MI AND wander IS POK) THEN DOIT

21: IF buffer IS NL (AND freq $=N L \rightarrow$ d_buffer is MI AND wander IS POK) THEN DOIT

22: IF buffer IS NL (AND freq $=$ PL $\rightarrow$ d_buffer is MI AND wander IS POK) THEN DOIT

23: IF buffer IS NVL (AND freq $=$ PS $\rightarrow$ d_buffer is FI AND wander IS POK) THEN DOIT 24: IF buffer IS NVL (AND freq $=$ NS $\rightarrow$ d_buffer is FI AND wander IS POK) THEN DOIT 25: IF buffer IS NVL (AND freq $=$ PM $\rightarrow$ d_buffer is FI AND wander IS POK) THEN DOIT 26: IF buffer IS NVL (AND freq $=N M \rightarrow$ d_buffer is FI AND wander IS POK) THEN DOIT 27: IF buffer IS NVL (AND freq $=N L \rightarrow$ d_buffer is FI AND wander IS POK) THEN DOIT 28: IF buffer IS NVL (AND freq $=$ PL $\rightarrow$ d_buffer is FI AND wander IS POK) THEN DOIT

29: IF buffer IS PS (AND freq $=$ NS $\rightarrow$ d_buffer is SD AND wander IS POK) THEN DOIT 30: IF buffer IS PS (AND freq $=$ PS $\rightarrow$ d_buffer is SD AND wander IS POK) THEN DOIT 31: IF buffer IS PS (AND freq $=\mathrm{NM} \rightarrow$ d_buffer is SD AND wander IS POK) THEN DOIT 32: IF buffer IS PS (AND Ereq $=$ PM $\rightarrow$ d_buffer is SD AND wander IS POK) THEN DOIT

33: IF buffer IS PVI (AND freq = NS $\rightarrow$ d_buffer is MD AND wander IS POK) THEN DOIT 34: IF buffer IS PVL (AND freq $=$ PS $\rightarrow$ d_buffer is MD AND wander IS POK) THEN DOIT 35: IF buffer IS PVI (AND freq $=$ NM $\rightarrow$ d_buffer is MD AND wander IS POK) THEN DOIT 36: IF buffer IS PVL (AND freq $=$ PM $\rightarrow$ d_buffer is MD AND wander IS POK) THEN DOIT 37: IF buffer IS PVL (AND freq $=N L \rightarrow$ d_buffer is MD AND wander IS POK) THEN DOIT 38: IF buffer IS PVL (AND freq $=$ PL $\rightarrow$ d_buffer is MD AND wander IS POK) THEN DOIT

39: IF buffer IS PL (AND freq $=$ NS $\rightarrow$ d_buffer is FD AND wander IS POK) THEN DOIT 40: IF buffer IS PL (AND freq $=$ PS $\rightarrow$ d_buffer is FD AND wander IS POK) THEN DOIT 41: IF buffer IS PL (AND freq $=\mathrm{NM} \rightarrow$ d_buffer is FD AND wander IS POK) THEN DOIT 42: IF buffer IS PL (AND Ereq $=$ PM $\rightarrow$ d_buffer is FD AND wander IS gOK) THEN DOIT 43: IF buffer IS PL (AND freq $=N L \rightarrow$ d_buffer is FD AND wander IS POK) THEN DOIT 44: IF buffer IS PL (AND freq $=$ PL $\rightarrow$ d_buffer is FD AND wander IS POK) THEN DOIT

The third and fourth rule clusters deal with constraint satisfaction. The third cluster takes action only when MTIE is in danger of violation. It also includes rules to bring MTIE back into compliance if it should ever increase beyond the mask.

45: IF wander IS BAD (AND freq $=2 \rightarrow$ wander IS POK) THEN DOIT

46: IF wander IS BAD (AND freg = PS $\rightarrow$ wander is POK) THEN DOIT

47: IF wander IS BAD (AND Ereq $=$ NS $\rightarrow$ wander is POK) THEN DOIT

48: IF wander IS BAD (AND freq $=$ NM $\rightarrow$ wander is POK) THEN DOIT

49: IF wander IS BAD (AND freq $=$ PM $\rightarrow$ wander is POK) THEN DOIT

50: IF wander IS BAD (AND freq $=$ PL $\rightarrow$ wander is POK) THEN DOIT 
51: IF wander IS BAD (AND freq $=\mathrm{NL} \rightarrow$ wander is POK ) THEN DOIT

52: IF wander IS VIOLATED (AND freq $=$ PM $\rightarrow$ wander is BETTER) THEN DOIT

53: IF wander IS VIOLATED (AND freq $=$ NM $\rightarrow$ wander is BETTER ) THEN DOIT

54: IF wander IS VIOLATED (AND freq $=$ PL $\rightarrow$ wander is BETTER) THEN DOIT

55: IF wander IS VIOLATED (AND freq $=\mathrm{NL} \rightarrow$ wander is BETTER) THEN DOIT

56: IF wander IS VIOLATED (AND freq = PMax $\rightarrow$ wander is BETTER) THEN DOIT

57: IF wander IS VIOLATED (AND freq $=$ NMax $\rightarrow$ wander is BETTER ) THEN DOIT

Finally, the fourth cluster of rules are invoked when the buffer is in danger of violation. The first rule applies when the buffer is positive and too large, yet is still increasing. In this case, a maximum allowed frequency offset is applied so long as it does not violate the wander specifications.

58: IF buffer IS P2L AND d_buffer IS POS (AND freq $=$ NMax $\rightarrow$ wander is POK) THEN DOIT 59: IF buffer IS N2L AND d_buffer IS NEG (AND freq $=$ PMax $\rightarrow$ wander is POK) THEN DOIT 


\section{CHAPTER VII}

\section{SIMULATION ENVIRONMENT}

\section{A. SIMULATION FRAMEWORK}

The controller described in the previous chapter was implemented and incorporated into the simulation environment illustrated in Figure 29.

The simulator consists of a set of C++ [39] objects which are coordinated by a custom discrete-event simulation engine. Parameters are passed to the simulator through a database object and various custom files. After the simulation is completed, samples of pertinent quantities are written to files and illustrated by a motif-based graphical display and browser.

\section{B. VALIDITY}

The validity of our results depend strongly on the validity of our simulation engine and simulation models. Therefore great care was taken to ensure that each model accurately reflected the system, signal, or format it was designed to represent. Still, a physically realized implementation might exhibit slightly different waveforms, just as no two physical systems of this complexity would be identical.

Fortuitously, our simulator has one significant advantage over simulators of other types of systems: It is a digital program simulating a largely digital system. Specifically, the input is a digital signal (albeit a continuous-time one), the PKBC algorithm internal to the simulator is the very same program that would be recompiled for a realworld implementation, and the output frequency generator is a purely digital system 


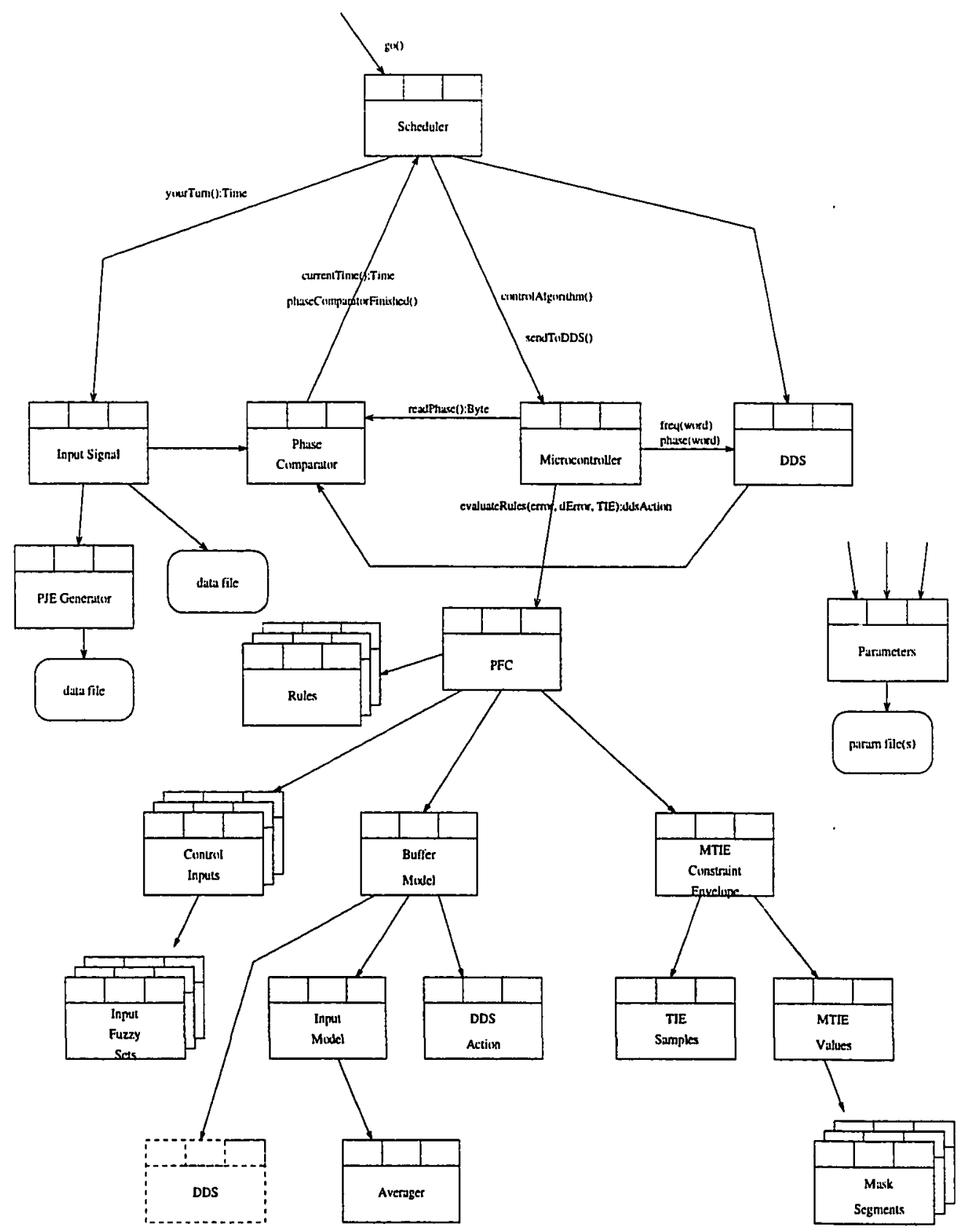

Figure 29. Simulator Object Interaction Diagram

coupled with a notch filter and hard limiter. 


\section{SIMULATION MODULES}

Here we describe the components of the simulator which are not part of the PKBC algorithm, but instead allow the algorithm to function in a virtual environment.

\section{Scheduler}

The scheduler schedules discrete-time events at the module level. Specifically, the input and microcontroller modules (described below) are invoked by the scheduler at the requested time. Then, when each module returns, it informs the scheduler of the time at which the next call is to occur. The scheduler exits when the terminal simulation time is reached.

\section{Input Signal}

The input signal module emulates a DS3 signal after overhead and stuff-bytes have been removed. To do this, the nominal (pre-PJE) DS3 rate, the STS-1 synchronization frequency, and the structure of the DS3 to STS-1 mapping are made available at runtime. During early development, the input was modeled at the clock level (each rising and falling edge), but it quickly became evident that such fine time resolution was both unnecessary and extremely computation intensive. At nominal data rates, the scheduler was required to call the input module $2 \cdot 44,736,000$ times for each second of simulation. Instead, we calculate the cumulative effect of an entire STS-1 row, resulting in an execution-time reduction of several orders of magnitude. After each row, the number of DS3 data bits in the row (621 or 622 , depending on the stuff bit) is sent to the phase comparator.

\section{PJE Generator}

All PJEs are generated by the PJE module. For maximum flexibility, a method of specifying arbitrary PJE profiles was developed. PJEs may be specified at arbitrary 
times and/or at arbitrary rates and with positive or negative polarities - within the constraints of the SONET standard. The PJE input file may contain any number of entries of the following form:

$$
\text { startTime TimeBetweenPJEs Polarity }
$$

where the startTime supersedes the previous TimeToNextPJE.

If an indefinite sequence of positive PJEs is desired, beginning at a simulation time of 3 seconds and with an interval of 1 second, the PJE file would contain the single line:
3.0
1.0
1

If each PJE is to be placed at irregular times, the startTime field may be assigned the desired time of each PJE and TimeToNextPJE may be set to a number larger than the length of the simulation. Most profiles use a combination of the above two techniques.

Although the PJE generator provides precise timing information during simulation, such precision would not be available in a real-world implementation. We anticipate this lack of time resolution to have only negligible effect, however, since PJE scenarios in Chapter VIII actually simulate missing or extra PJEs (as many as four missing PJEs) while still meeting jitter specifications.

\section{DDS Output}

The DDS module models the operation of a DDS. It accepts frequency and phase tuning words from the microcontroller object, and calculates the output frequency that will result. When asked, it can give the number of output clock cycles since the last time it was queried. The length of $T W_{F}$ is limited to the number of bits used in internal integer calculations of the simulating microprocessor (32, in our case), since native arithmetic operations are used to calculate the output of the DDS. This limitation could be removed through the use of an arbitrary-precision library, but at a cost of increased 
simulation time.

\section{Phase Comparator}

The phase comparator was initially designed to model a physical phase comparator we had designed and constructed. This phase comparator was able to make both integer and fractional-cycle measurements. However, we found that sufficient resolution was achieved by sampling the integer phase difference after every STS-1 row and averaging over a large number of samples.

The physical implementation of the simplified phase comparator, shown in Figure 30

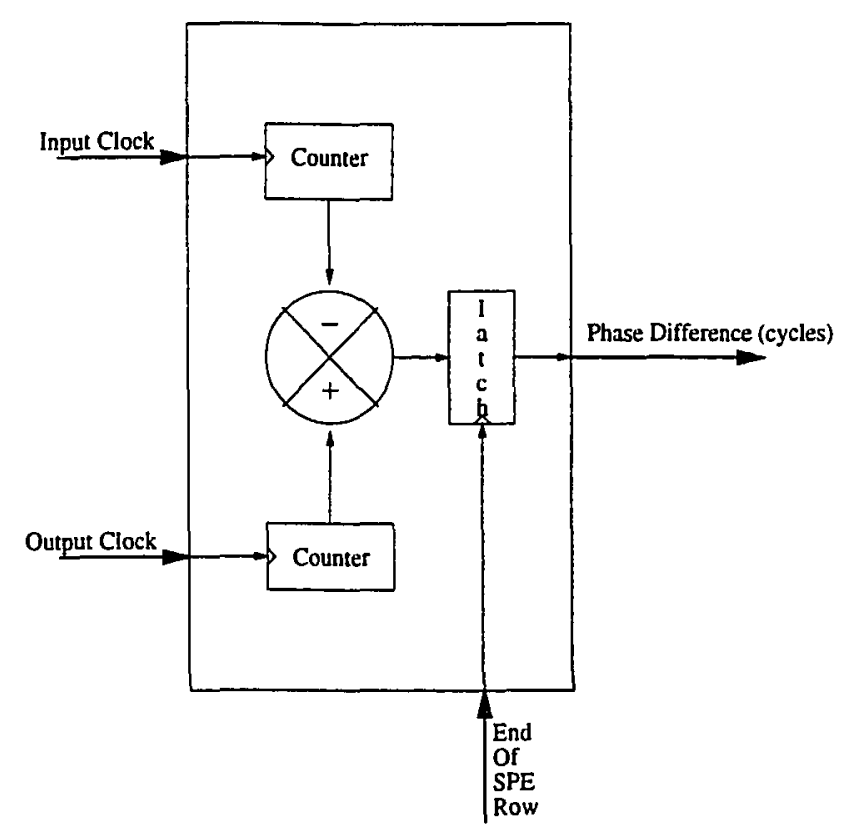

Figure 30. Phase Measurement

is analogous to an up-down binary counter with the count-up input connected to the read-clock and the count-down input connected to the write-clock of the elastic store. 


\section{SIMULATION MEASUREMENTS}

\section{TIE Reference Clock}

The Time Interval Error measure, described previously, is necessary for the calculation of both wander and jitter and requires a frequency reference $f_{D S 3}$. For jitter, longterm stability of the reference clock is not necessary since low frequencies are attenuated by the high-pass filter.

For the MTIE calculation, however, long-term stability of the reference clock is important since it is against this clock that the long-term variation of the outgoing DS3 signal will be measured. The question that arises is: If the reference against which TIE is measured is imperfect, would external equipment testing MTIE compliance reach the same conclusion as the control algorithm? The answer to this question is found in the definition of DS3 MTIE [4]. The specified mask assumes that TIE samples are taken relative to a system clock readily available to the desynchronizer. Thus the same clock that would be used by an external MTIE measurement device is available to our PKBC algorithm. If this were not the case, a clock with sufficient accuracy would be required such that the TIE samples used to generate the MTIE Constraint Envelope would yield an acceptable margin of error.

\section{Jitter Measurement}

The rate at which TIE is sampled for calculating jitter is not bounded by the controller's update rate $T_{s}$ (although our algorithm restricts it to an integer multiple of $T_{s}$ ). In this way, the peak-to-peak jitter error resulting from the discrete-time measurement (as derived in Chapter III) may be reduced to whatever levels are deemed acceptable. We operated at a TIE sample rate $\left(T_{T I E}\right)$ of $200 \mathrm{~Hz}$, which was shown to result in a jitter measurement error of less than $1 \%$. 
Jitter measurement is included in the simulator for testing purposes. It is not specifically used by the controller and may be omitted in a hardware implementation.

\section{Wander}

Although we represent wander by the MTIE measure, we do not calculate MTIE explicitly. Rather, the MTIE Constraint Envelope is generated as a function of both the MTIE mask and all previous TIE measurements (to the limits of the mask) as described in the previous Chapter. Since MTIE is undefined for observations times less than 0.1 second, the envelope is recomputed at a rate of $10 \mathrm{~Hz}$. When the simulation is complete, a single plot showing the generated TIE and the MTIE mask may be displayed for the user.

\section{E. GRAPHICAL USER INTERFACE}

An important part of the simulation environment is our custom graphical user interface. Any internal variable may be sampled and stored by a set of Signal objects. These storage objects are given samples at regularly spaced intervals with the member function:

[Object Name].dataIn(Value, Time)

After the simulator reaches the terminal time, another object, called the GraphWindow collects all the Signals and asks each to display itself within a PanedWindow Motif widget which allows vertical scaling of any signal of choice. At the highest level, the XInterface object manages the Panedwindow, several pushbuttons for quitting or writing the signals to their appropriate files, and the fuzzy sets illustration window described below.

If the mouse button is pressed while the cursor is in the signals area, a vertical bar cursor is displayed at the relevant time for each signal, and the value at the cursor 
is displayed along with other relevant information. Simultaneously, the fuzzy sets below are illuminated with the values at the cursor. If, for example, the cursor shows the buffer with a value of 12 , a tick mark is drawn on the $\mathrm{x}$-axis of the buffer fuzzy sets, and the non-zero truth value of each fuzzy set is illustrated with a colored, dotted line. In addition, if the action taken under the cursor is predicted to effect a change in any of the fuzzy variables, both current and predicted values are shown with a directed arc from the current value to the predicted value. This feature may be seen in Figure 31. The arrow indicates the d_buffer which, using prediction, is expected the next sample. As can be seen from the clipped snapshot of Figure 32, the predicted location is realized. The cursor may be moved by a single or multiple points using the left and right arrow keys and numeric multipliers. Also, the actual MTIE constraint envelope and the predicted TIE plot are written to files for concurrent display.

A few words of explanation may be needed for the two simulation snapshots. The first plot is of the "Input Frequency". This is the nominal frequency of the incoming DS3 signal (minus the effect of any PJEs). The "DDS Frequency Output" is the next plot, and may be seen to change by $0.5 \mathrm{~Hz}$ at the cursor (this corresponds to a "Frequency Effort" of $0.5 \mathrm{~Hz}$ ). The "Buffer" plot shows the number of bits in the buffer, and so on. Near the bottom of the snapshot, the fuzzy sets are shown and illustrated as described above.

If the user desires to view one graph in greater detail, he may either click the "Write" button and then view the resulting data file with a separate plotting program or use the mouse to expand the specific signal's window which causes the plot to be scaled automatically. In addition, outliers may be ignored from consideration. Horizontal scaling is also possible. 


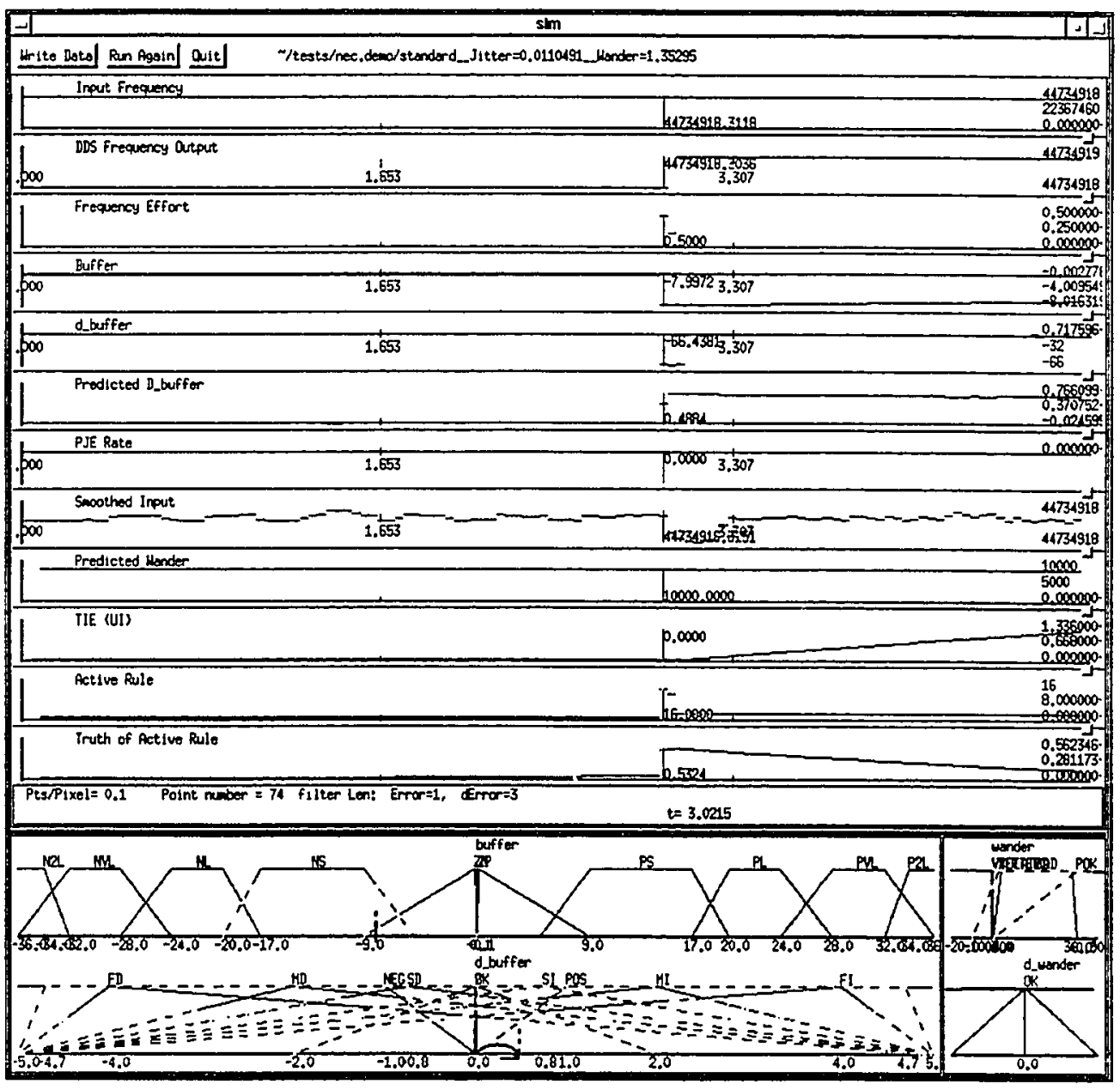

Figure 31. Simulation Example Showing Predicted d_buffer movement 


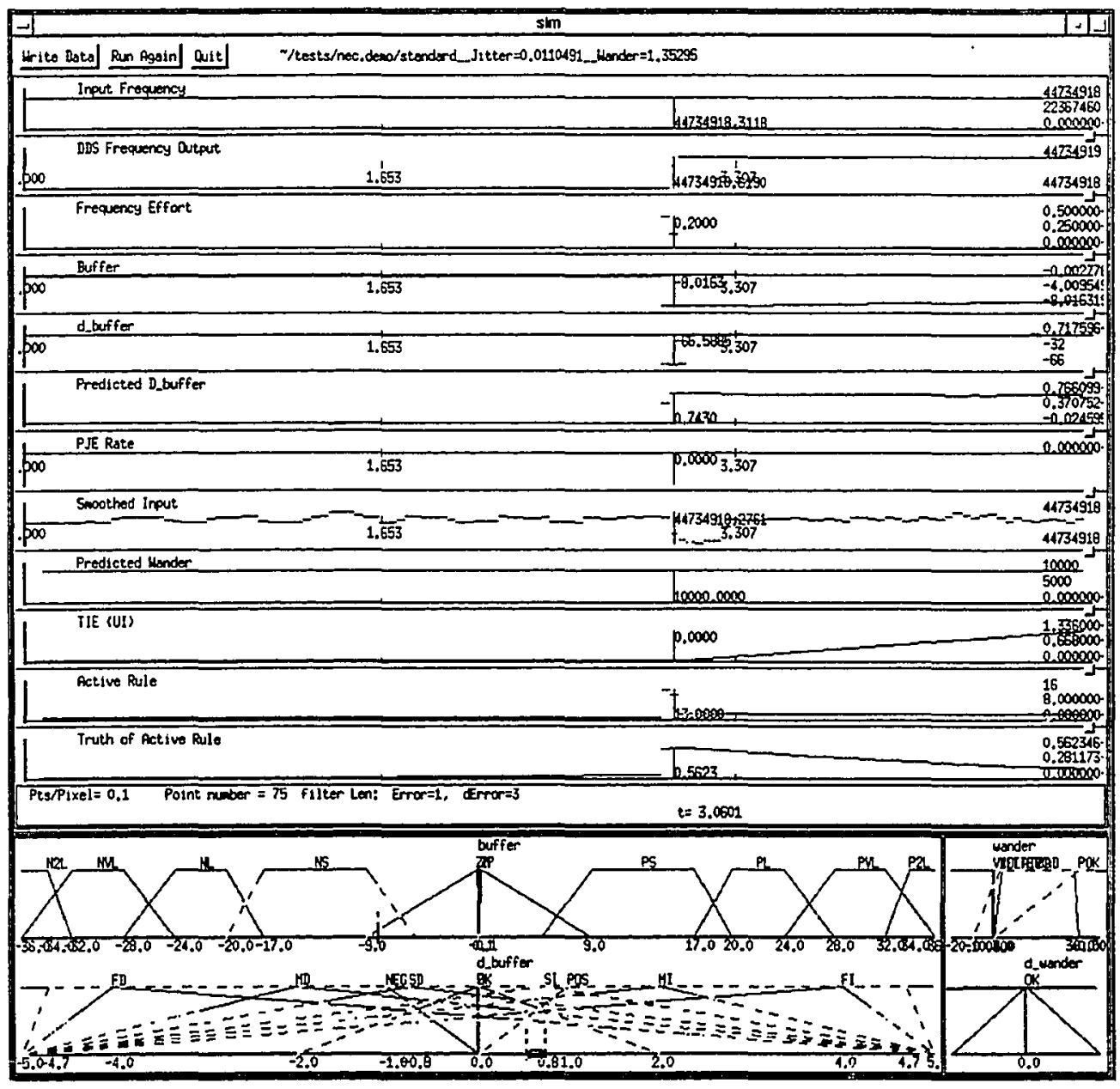

Figure 32. Simulation Example Showing d_buffer movement 


\section{F. COMPUTATIONAL COMPLEXITY}

The time required to calculate one iteration of the control loop is an important consideration when implementing any computational control algorithm. While it is not likely that the PKBC algorithm would account for $100 \%$ of the computations of a realworld control loop, a study of its computational demands can be useful.

In this section we consider the change in computational load as various control system parameters are adjusted. We use the commonly used $\operatorname{Order}$ operator $(O())$ [10] to denote the asymptotic behavior of the function relative to the parameter under consideration. We then proceed to estimate the constants associated with each significant variable. Since the execution time of the algorithm is linearly dependent upon the number of times it is called per second $\left(1 / T_{s}\right)$, this parameter is $O(n)$. The number of terms and the number of rules are each $O(n)$, resulting in a combined rule-term complexity of $O\left(n_{\text {rules }} \cdot n_{\text {terms. }}\right)$ since each term is considered independently.

With the above asymptotic complexities of the PKBC and knowledge of other processes in the simulator itself, we arrived at an expression which characterizes the time necessary to complete a simulation of the control system;

$$
t=K_{S t u n U p}+T_{\text {sim }}\left(K_{O H}+\frac{1}{T_{s}} \cdot K_{P K B C}\right)
$$

$K_{\text {Surt }}$ is the time needed to read configuration files and other necessary tasks when the simulator is invoked (assuming a moderate number of rules and terms). $T_{\text {sim }}$ is the desired terminal simulation time, in seconds. $K_{O H}$ is overhead of the simulator which is independent of the sample time of the control system. This includes the generation of the input signal, PJEs, and other scheduling overhead. $T_{s}$ is the sample period of the control system and $K_{P K B C}$ is the time required for each iteration of the control loop. 
$K_{P K B C}$ may be further expressed as

$$
K_{P K B C}=K_{\text {Rule }} * N_{\text {Rules }}+K_{R \& T}\left(N_{\text {Rules }} \cdot N_{\text {Terms }}\right)+K_{\text {misc }}
$$

where $K_{\text {Rule }}$ is the computation time associated with the evaluation of each rule, $K_{R \& T}$ is the time constant associated with each term of each rule, $N_{\text {Rules }}$ and $N_{\text {Terms }}$ are the number of rules and terms per rule respectively, and $K_{\text {misc }}$ includes all other PKBC computations.

Generation of the MTIE envelope has a theoretical computational complexity of $O\left(\log _{\text {buse }} n_{\text {env }}\right)$ where base is the exponential growth constant of the MTIE constraint envelope (typically about 1.5) as described in Chapter 4 and $n_{e n v}$ is the desired length of the constraint envelope in units of $T_{s}$. This term is not included in the expression for $T_{P K B C}$ since its effect on simulation time was too small to be measured with confidence and is therefore included in $K_{\text {misc }}$.

The first two terms of $T_{P K B C}$ and empirical measurements are plotted together in Figure 33. Obviously, the model accurately reflects measurements of simulation time for widely varying numbers of rules and terms.

The constants of Equations (5) and (6) are listed in Table III. They were derived through timed simulations and in each case the linearity of the expression was verified by multiple simulations.

The platform used for the simulations was a $90 \mathrm{MHz}$ Pentium-based IBM compatible computer with 32 Megabytes of RAM and running Linux version 1.2.13. The compiler was gcc/g++ version 2.6 .3 with optimization level four. Assuming similar processing power, a single iteration of a 60 -rule, 4 -term controller would require $2.14 \mathrm{~ms}$, far less than the $T_{s}$ of $40 \mathrm{~ms}$ used in our tests. It is also important to notice that $K_{R u l e}, K_{R \& T}$ and $K_{\text {Misc }}$ include the time necessary to collect data in Signal objects for later display, execute debug statements and conditionals, and additional time due to algorithms which 


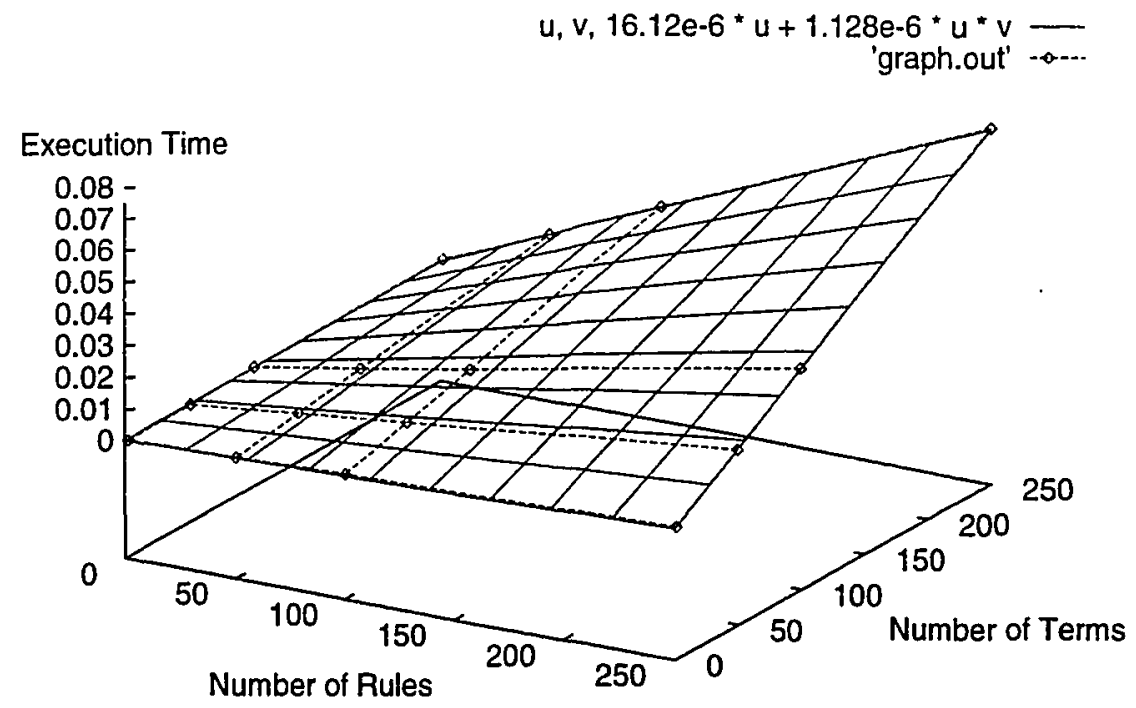

Figure 33. Rule and Term Execution Times

TABLE III

Computational Complexity Multipliers

\begin{tabular}{|l|c|}
\hline \multicolumn{2}{|c|}{ Time Constants } \\
\hline Name & Value \\
\hline$K_{\text {Sturi }}$ & 0.57 \\
\hline$K_{O H}$ & 0.48 \\
\hline$K_{\text {Rule }}$ & $16.12 \mathrm{e}-6$ \\
\hline$K_{\text {R\&T }}$ & $1.128 \mathrm{e}-6$ \\
\hline$K_{\text {Misc }}$ & $906.0 \mathrm{e}-6$ \\
\hline
\end{tabular}

are optimized for readability and maintenance rather than speed. One example of this is the repeated calculation of predicted buffer levels for each candidate action in the rulebase (60 times in the above example) even though there are only 11 possible output values. 


\section{CHAPTER VIII}

\section{TESTING AND EVALUATION}

This chapter presents the tests used to verify that the PKBC meets our two primary goals. Our first goal was to verify that the controller produced acceptable (or better) jitter in the normal case as a result of PJE profiles specified in [3]. The first set of tests verify this claim. However, compliance with the standard tests is only part of the capabilities of the controller (albeit a necessary part) since other desynchronization techniques have also been shown to meet this requirement [15].

The second goal was to study the PKBC's ability to maintain MTIE compliance and prevent buffer overflow or underflow for as iong as possible. With both constraints facing the danger of violation, we suggested that the algorithm would be capable of satisfying one constraint at the expense of the other as determined beforehand by the designer. This is the larger challenge. The performance of the PKBC is demonstrated in a second set of tests.

\section{A. STANDARD JITTER TESTS}

To facilitate the testing of jitter performance, we created a directory structure where the parameters file, rules, fuzzy sets, and MTIE mask could all be defined in one place. Subdirectories for mapping, standard, burst, phase transient burst, and degraded modes each included shell and Awk [40] scripts for automatically setting up experiments and generating PJE files.

For example, a pair of files in the degraded/continuous subdirectory specify the 
values of $t$ (rate of continuous PJEs) and the values for $T$ (the rate of spurious PJEs) desired for the experiment. If $T$ is defined by the set $\{30,35,40,60\}$ and $t$ is defined by the set $\{0.034,0.05,0.1,0.5,1.0,5.0,10.0\}$, directories with names derived from each $T$ value are created and in each of them, a directory is created for each of the $t$ values (for a total of 24 directories in this example). Finally, in each of these lowest directories, a custom PJE generator writes the appropriate PJE profile file.

A simulation was run in each of the directories for 10 repetitions of the most infrequent event and the resulting TIE samples were written to a file for future processing. We found that this number of repetitions was sufficient to reveal the amount of jitter that would result from the PJE input pattern. The jitter generated before the first few spurious PJEs was ignored as specified in the standard by the Initialization and Cool Down periods. If a change is made to a global file such as the rule or fuzzy set files, a single command creates all the appropriate directories, generates the PJE profiles, runs the simulations, calculates the resulting jitter, and automatically generates the representative tables and graphs.

In the following sections we show the jitter generated by the various PJE profiles defined in [3]. Desynchronization equipment must pass these standardized tests for certification.

\section{Mapping Jitter}

In the absence of all other type of jitter, the desynchronizer must generate less than 0.4UI of jitter resulting from the mapping process and pulse stuffing. We found that, in the absence of PJEs, the PKBC consistently generated less than 0.03UI of jitter for various stuffing ratios.

Some jitter is unavoidable. Since the frequency range of the DDS is quantized, the finite frequency mismatch between the input and the output requires that the output 
be dithered to achieve the same average frequency as the input.

Figure 34 shows a representative simulation snapshot. The simulator's display is described in Section E of Chapter VII. Table IV and Figure 35 show the jitter which results from various stuffing ratios.

\section{TABLE IV}

Mapping Jitter vs. Stuffing Ratio

\begin{tabular}{|l|c|}
\hline \multicolumn{2}{|c|}{ No PJEs } \\
\hline $\begin{array}{c}\text { Stuffing } \\
\text { Ratio }\end{array}$ & $\begin{array}{c}\text { Jitter } \\
(\mathrm{UI})\end{array}$ \\
\hline \hline .01019 & 0.00410 \\
\hline .05123 & 0.00315 \\
\hline .1 & 0.01089 \\
\hline .1010 & 0.00315 \\
\hline .2 & 0.02257 \\
\hline .2012 & 0.00378 \\
\hline .3 & 0.01183 \\
\hline .3013 & 0.00331 \\
\hline .4 & 0.02478 \\
\hline .4014 & 0.00363 \\
\hline .41 & 0.00378 \\
\hline .5 & 0.02525 \\
\hline .5015 & 0.00331 \\
\hline .6 & 0.02478 \\
\hline .6016 & 0.00315 \\
\hline .7 & 0.01041 \\
\hline .7017 & 0.00378 \\
\hline .8 & 0.02478 \\
\hline .8018 & 0.00378 \\
\hline .9 & 0.00994 \\
\hline .9019 & 0.00378 \\
\hline .9901 & 0.00426 \\
\hline & \\
\hline
\end{tabular}




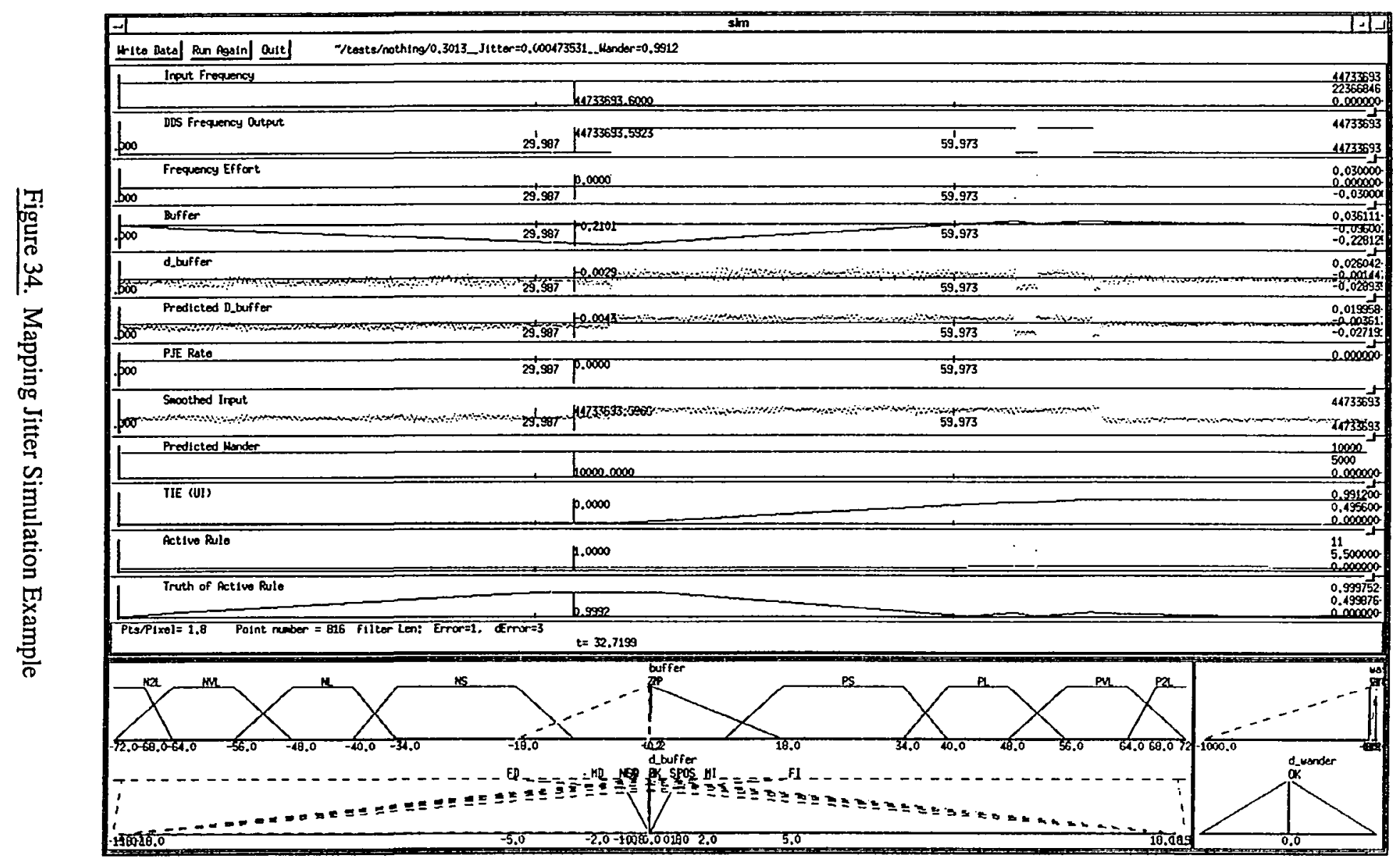




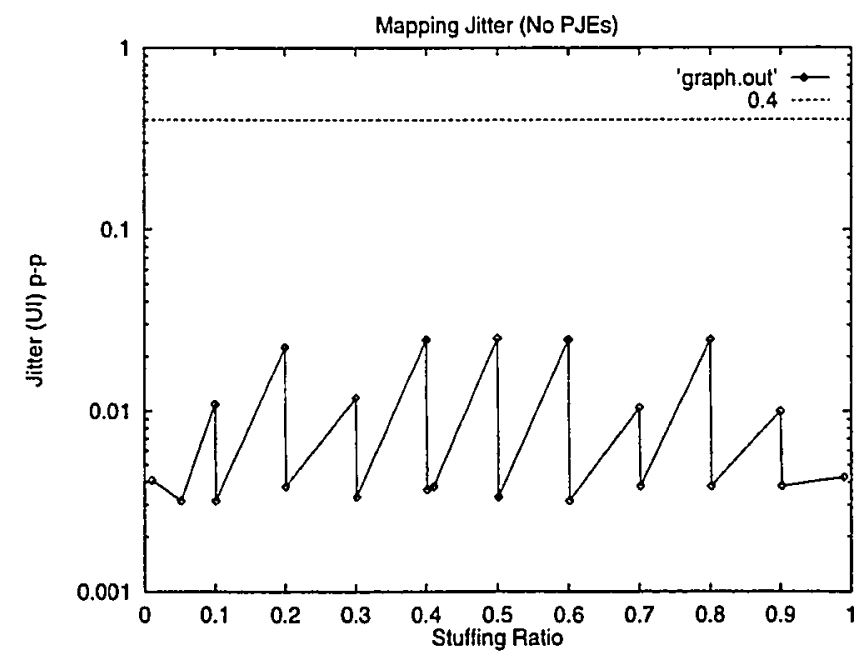

Figure 35. Mapping Jitter vs. Stuffing Ratio

Note that mapping jitter is far below the 0.4 UI allowed.

We found that stuffing ratios composed with small integers produced higher levels of jitter than ratios composed with larger numbers. For example, a stuffing ratio of $1 / 10=0.1$ results in more jitter than a ratio of $101 / 1000=0.101$. This can be attributed to the phase comparison method we employ. The additional digits in the stuffing ratio act as dither to increase the effective resolution of the phase measurements.

Although the existence of a sustained stuffing ratio of precisely $0 . r$ where $r$ is an integer from one and nine is possible, such a state would be rare in a real network. Still, mapping jitter performance even in this case would be greatly improved by using a better phase measurement scheme such as [35] or through additional filtering of the phase measurements.

The following jitter tests allow for mapping jitter of 0.4UI. However, since simulations show that our mapping jitter is very small, we subtracted $0.4 \mathrm{UI}$ from each of allowed jitter values below. It is expected that field tests of equipment employing this 
controller would show even greater jitter attenuation than is stated here. Since stuffing ratios are not specified in the standard, a ratio composed of large integers is used in the following tests.

\section{Standard Mode}

In addition to mapping jitter, an occasional pointer adjustment will be seen even in the standard mode. For this test, the time between spurious PJEs $(T)$ may range from 30 seconds to infinity. Figure 36 shows the official test.

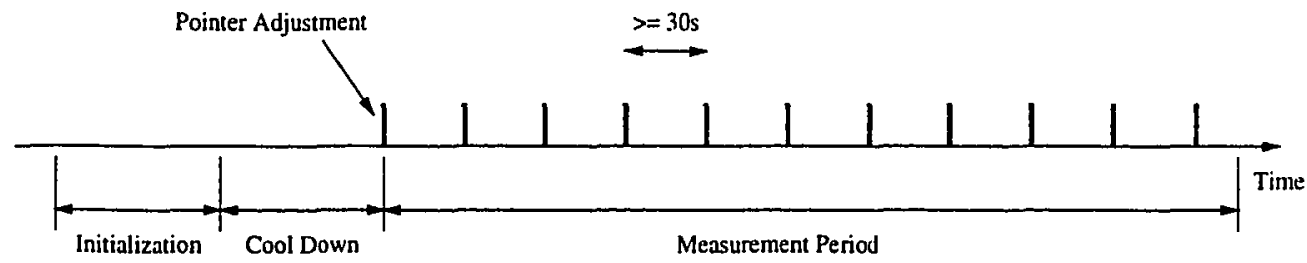

Figure 36. Standard Mode PJE Test Sequence

As can be seen from the sample simulation in Figure 37, phase transients resulting from the spurious PJE are eliminated within 30 seconds and thus an increase in $T$ does not significantly change the level of jitter generated. Jitter levels shown in Table $\mathrm{V}$ and illustrated in Figure 38 show little dependence upon the PJE rate. 


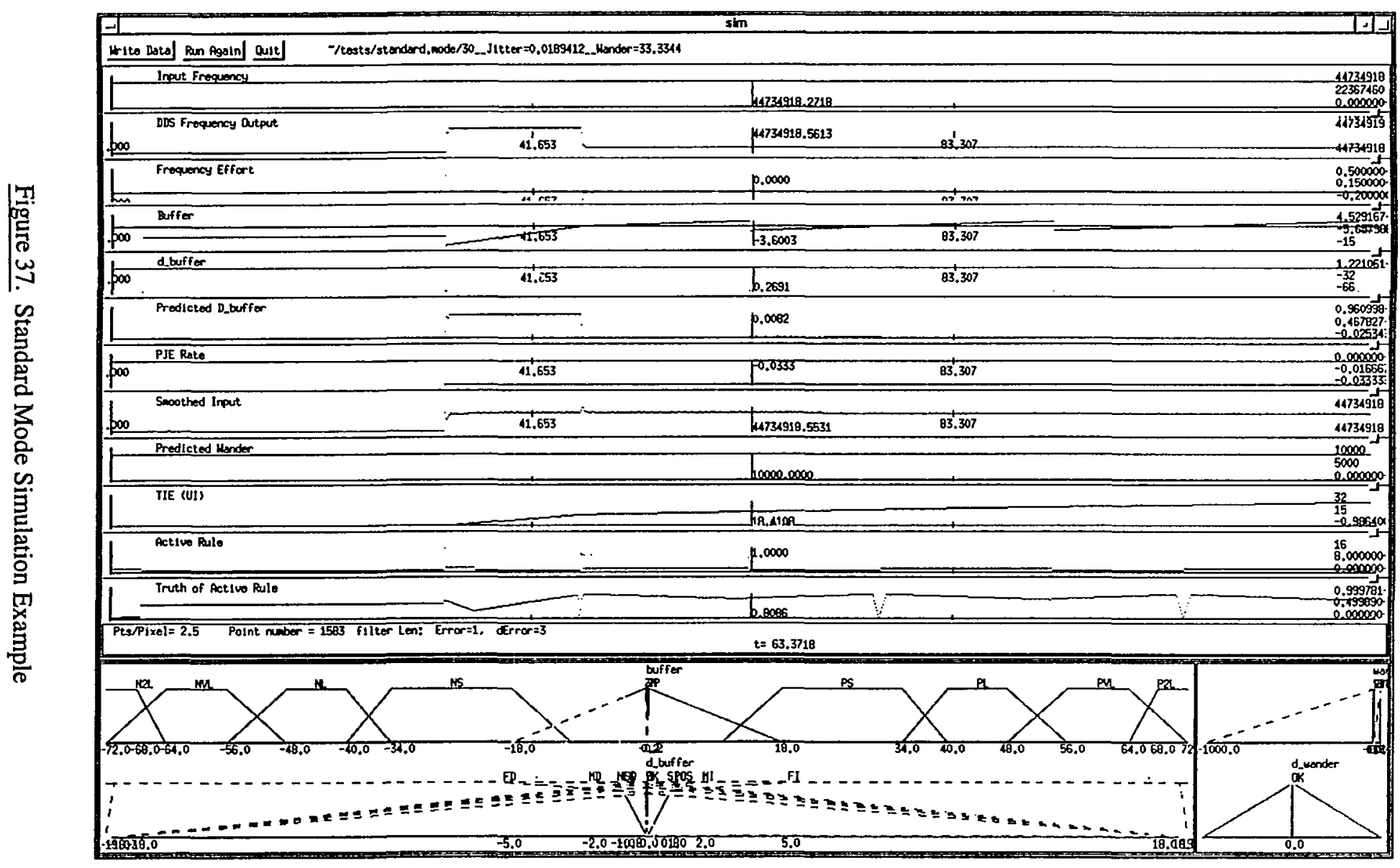


TABLE V

Standard Mode Jitter

\begin{tabular}{|c|c|}
\hline \multicolumn{2}{|c|}{ Standard Mode } \\
\hline $\begin{array}{c}\text { Time Between } \\
\text { Spurious PJEs (sec) }\end{array}$ & $\begin{array}{c}\text { Jitter } \\
\text { (UI) }\end{array}$ \\
\hline \hline 30 & 0.003 \\
\hline 35 & 0.003 \\
\hline 40 & 0.003 \\
\hline 50 & 0.003 \\
\hline 70 & 0.003 \\
\hline 100 & 0.003 \\
\hline
\end{tabular}

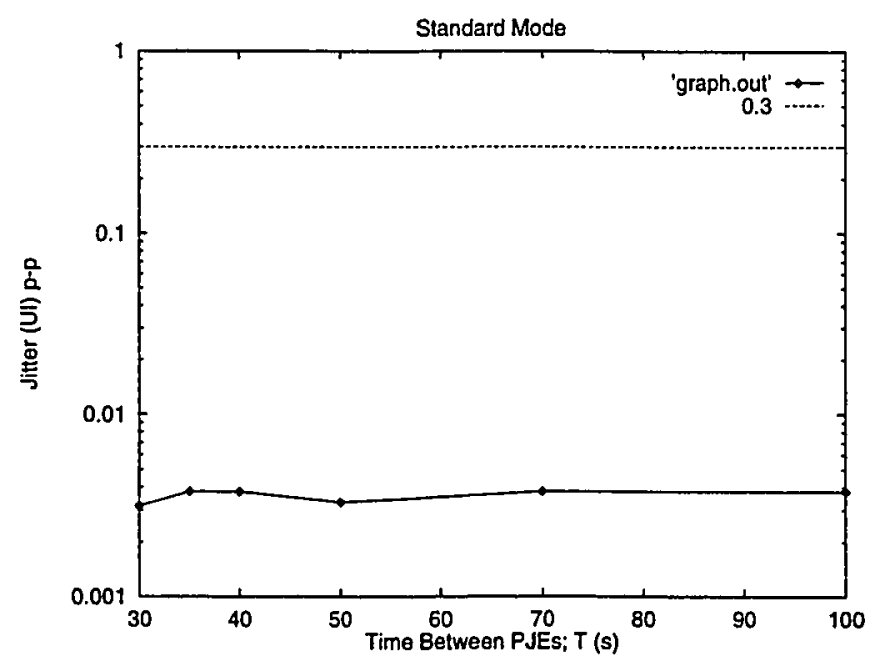

Figure 38. Standard Mode Jitter

Standard mode jitter is $12 \mathrm{~dB}$ below the $0.30 \mathrm{UI}$ specified.

\section{Burst Mode}

A PJE burst is composed of three PJEs separated by $0.5 \mathrm{~ms}$. The time between bursts $(T)$ ranges from 30 seconds to infinity, as illustrated in Figure 39.

Figure 40 shows a representative simulation with several bursts. Table VI and 


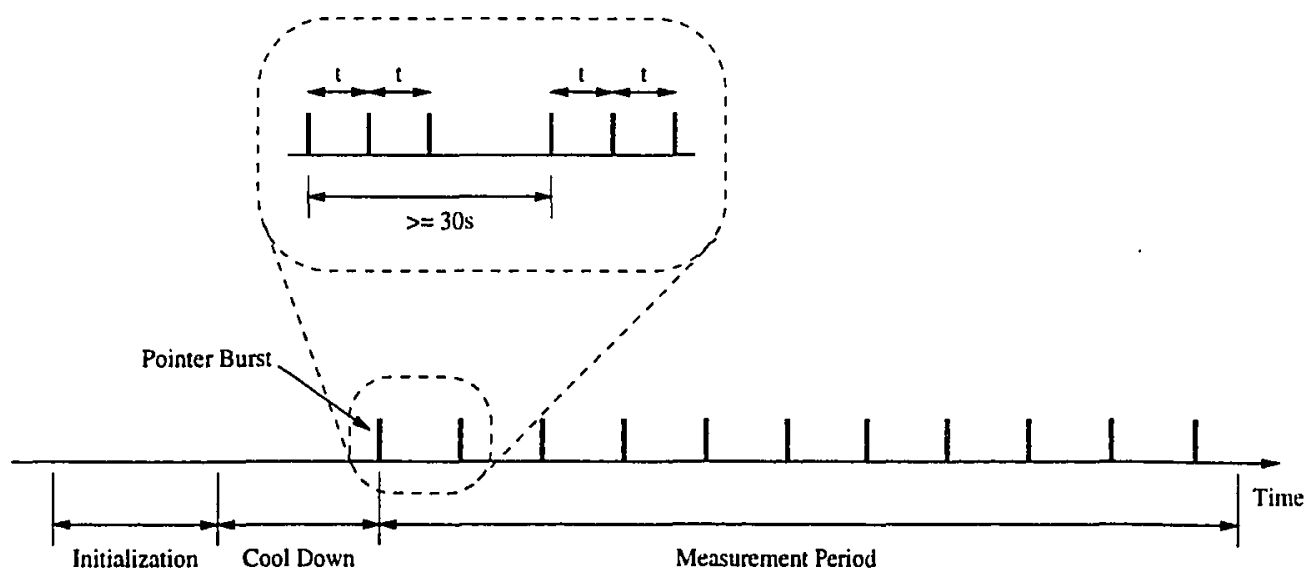

Figure 39. Burst Mode PJE Test Sequence

Figure 41 show that jitter has little dependence upon the time between bursts since in each case the buffer offset resulting from the burst is eliminated before the next burst.

TABLE VI

Burst Mode Jitter

\begin{tabular}{|c|c|}
\hline \multicolumn{2}{|c|}{ Burst Mode } \\
\hline $\begin{array}{c}\text { Time Between } \\
\text { PJE Bursts (sec) }\end{array}$ & $\begin{array}{c}\text { Jitter } \\
\text { (UI) }\end{array}$ \\
\hline \hline 30 & 0.019 \\
\hline 35 & 0.018 \\
\hline 40 & 0.019 \\
\hline 45 & 0.019 \\
\hline 50 & 0.019 \\
\hline 55 & 0.019 \\
\hline 60 & 0.018 \\
\hline
\end{tabular}




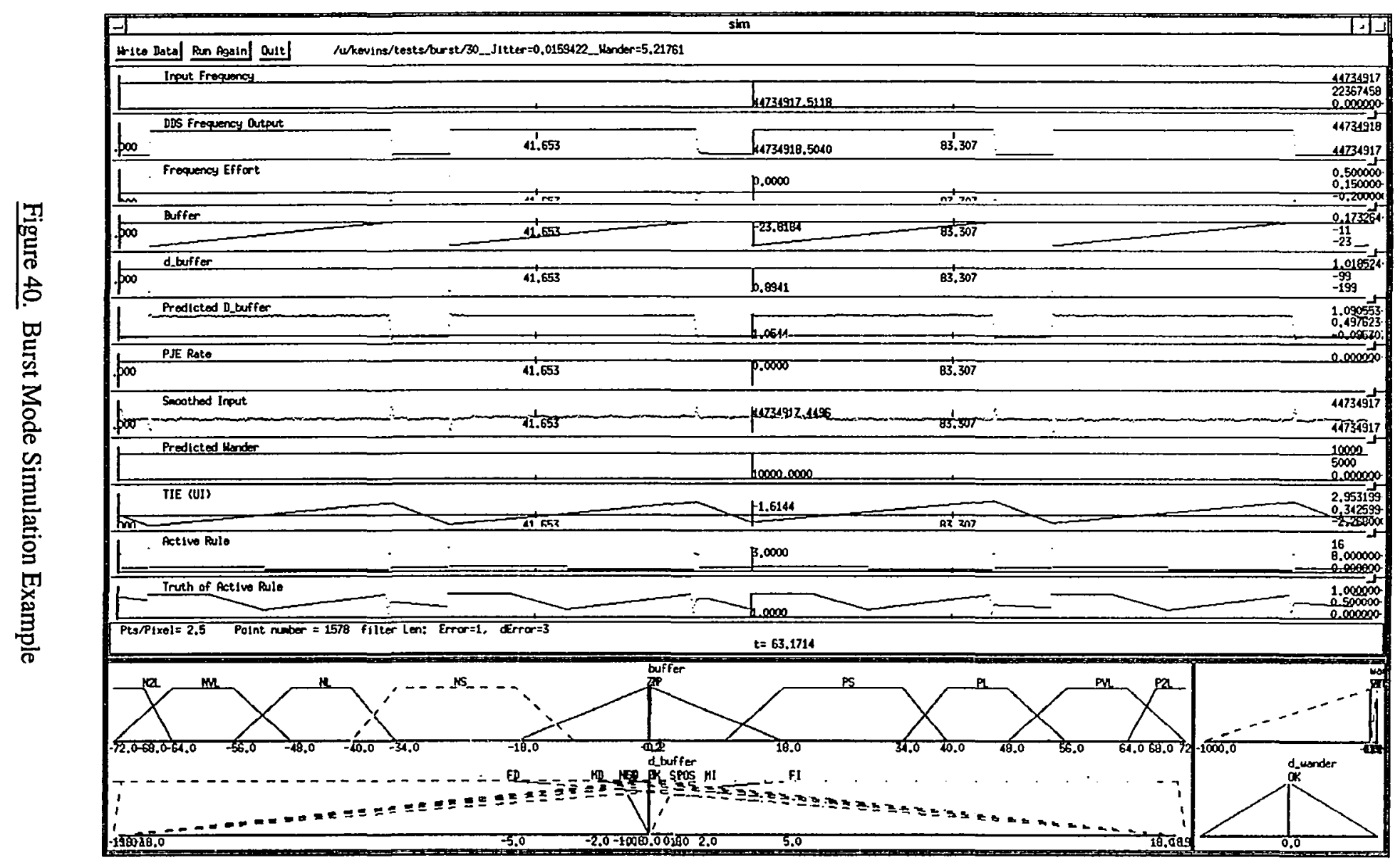




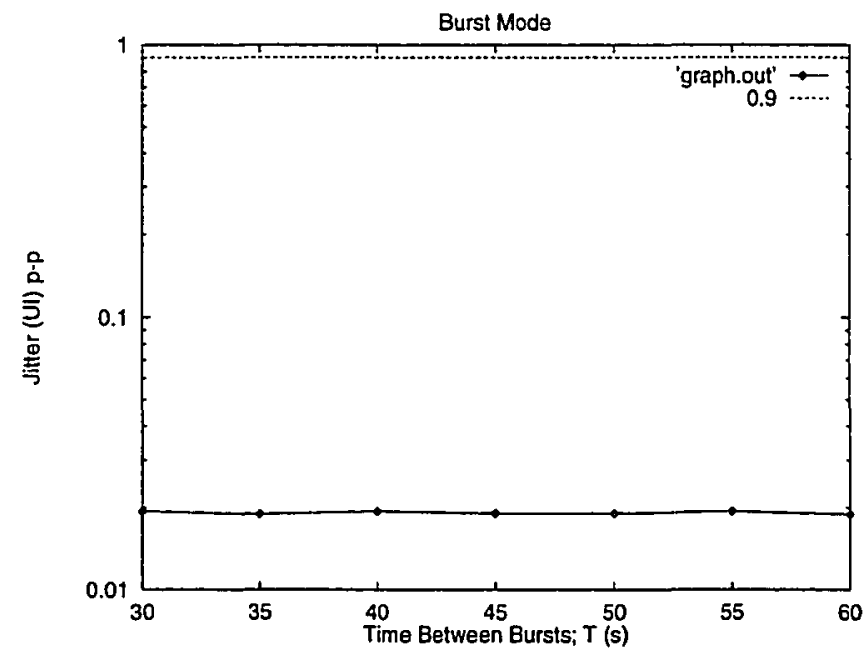

Figure 41. Burst Mode Jitter

Burst mode jitter is $16 \mathrm{~dB}$ below the $0.9 \mathrm{UI}(0.30 \mathrm{UI} \cdot 3 \mathrm{PJEs})$ specified.

\section{Phase-Transient Burst Mode}

A Phase-Transient burst is composed of seven PJEs in 2.5 seconds. The time between each burst $(T)$ is at least 30 seconds apart, as illustrated in Figure 42. Figure 43 shows a representative simulation. Table VII and Figure 44 show that there is little dependence upon the time between bursts, since each burst is eliminated within that time. 


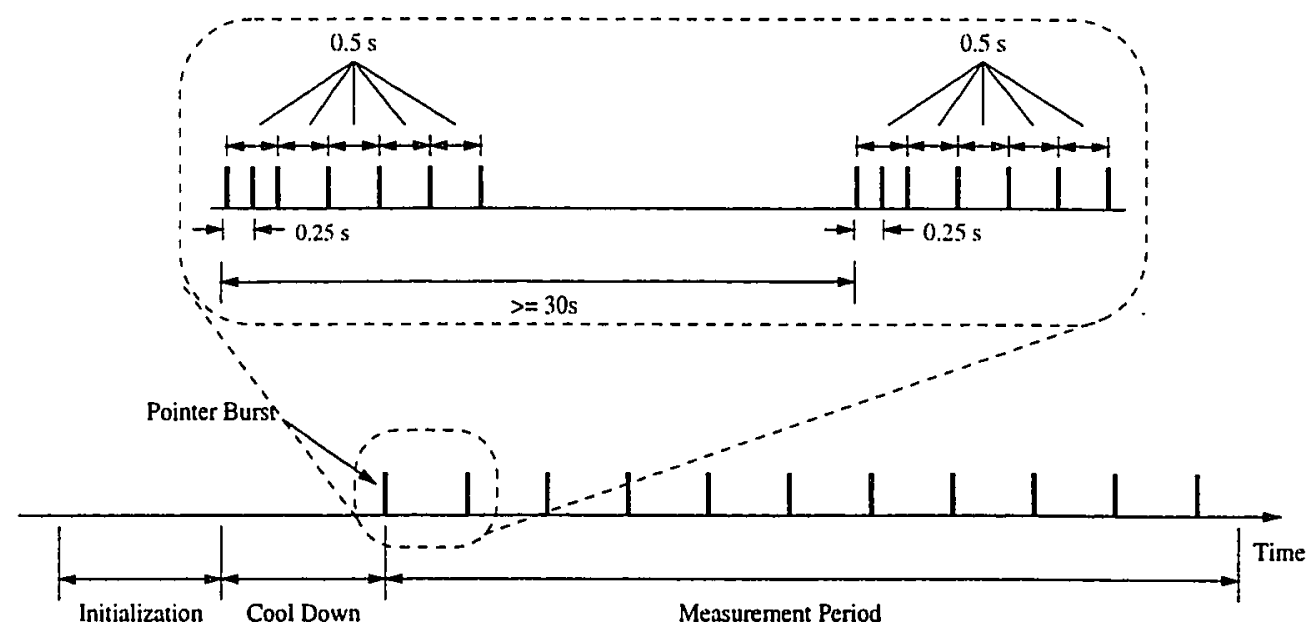

Figure 42. Phase-Transient Burst Mode PJE Test Sequence

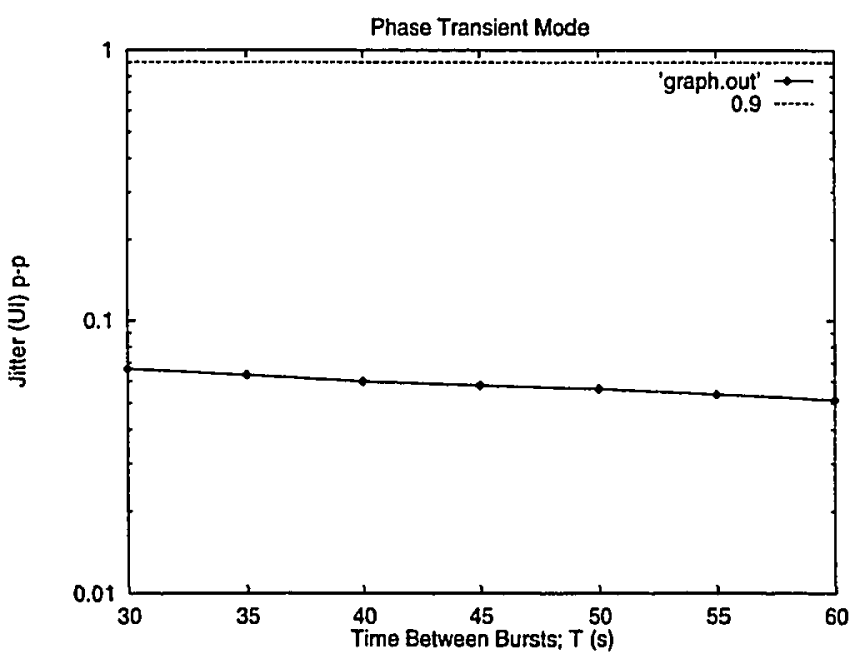

Figure 44. Phase-Transient Burst Mode Jitter

Phase-transient burst mode jitter is $16 \mathrm{~dB}$ below the $0.8 \mathrm{UI}$ specified. 


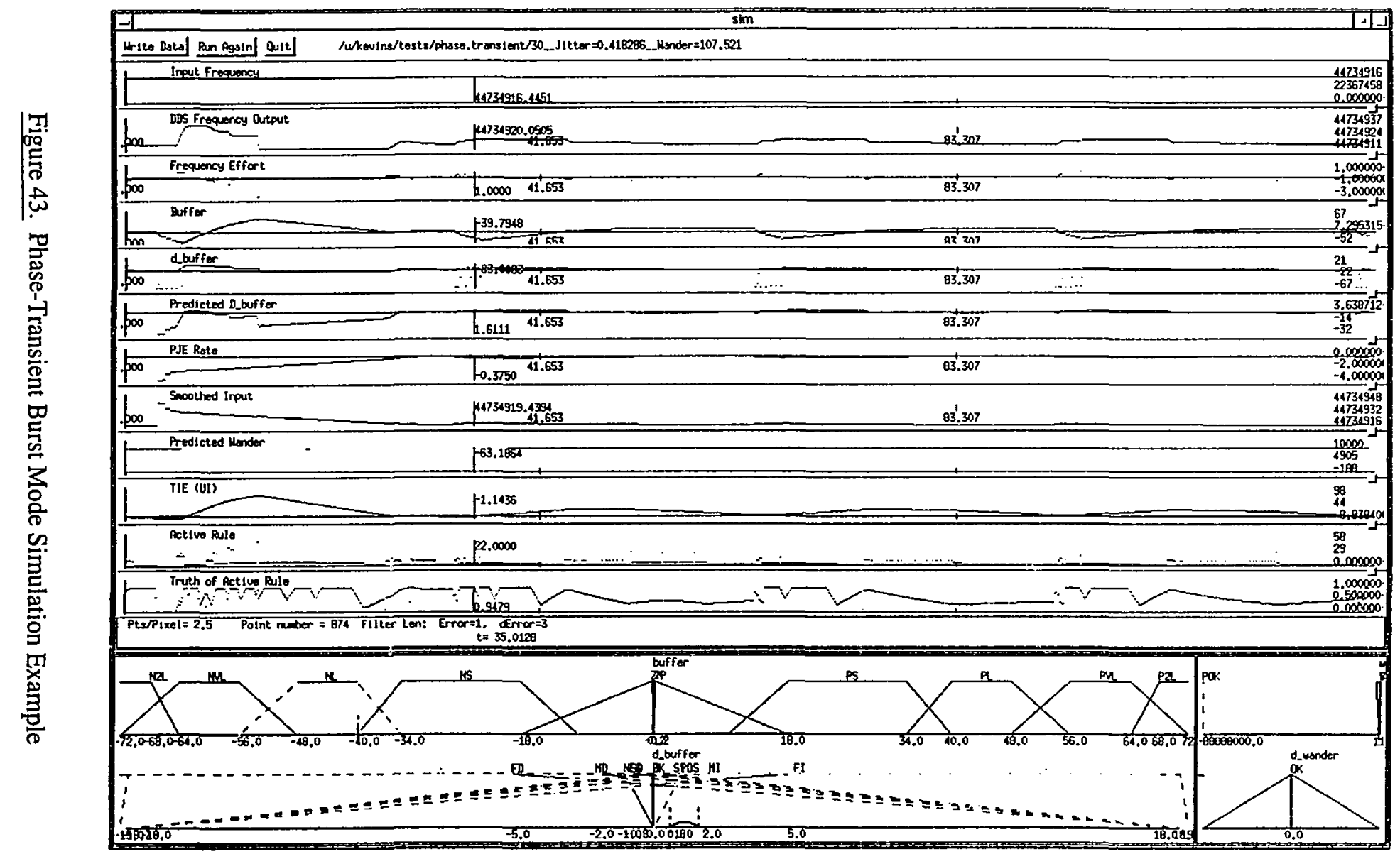


TABLE VII

Phase Burst Jitter

\begin{tabular}{|c|c|}
\hline \multicolumn{2}{|c|}{ Phase Transient Mode } \\
\hline $\begin{array}{c}\text { Time Between } \\
\text { PJE Bursts (sec) }\end{array}$ & $\begin{array}{c}\text { Jitter } \\
\text { (UI) }\end{array}$ \\
\hline \hline 30 & 0.066 \\
\hline 35 & 0.063 \\
\hline 40 & 0.059 \\
\hline 45 & 0.057 \\
\hline 50 & 0.056 \\
\hline 55 & 0.053 \\
\hline 60 & 0.051 \\
\hline
\end{tabular}

\section{Degraded Mode}

There are two separate PJE profiles for testing degraded mode jitter generation. The two profiles correspond to differences in implementation of the algorithm used to actually generate the PJEs. The first profile, shown in Figure 45 is called the "continuous pattern" and consists of a constant stream of PJEs augmented with standard-mode (spurious) PJEs every 30 seconds or more $(T)$. The time between the stream of PJEs $(t)$ ranges from $34 \mathrm{~ms}$ to $10 \mathrm{~s}$, and the distance between a continuous stream PJE and the spurious, standard-mode PJE, is $0.5 \mathrm{~ms}$. Again, separate tests are required for added and canceled pointers. Figure 46 shows a representative simulation. Table VIII and Figure 47 illustrate how jitter depends upon $T$ and whether the PJE is added or dropped. 

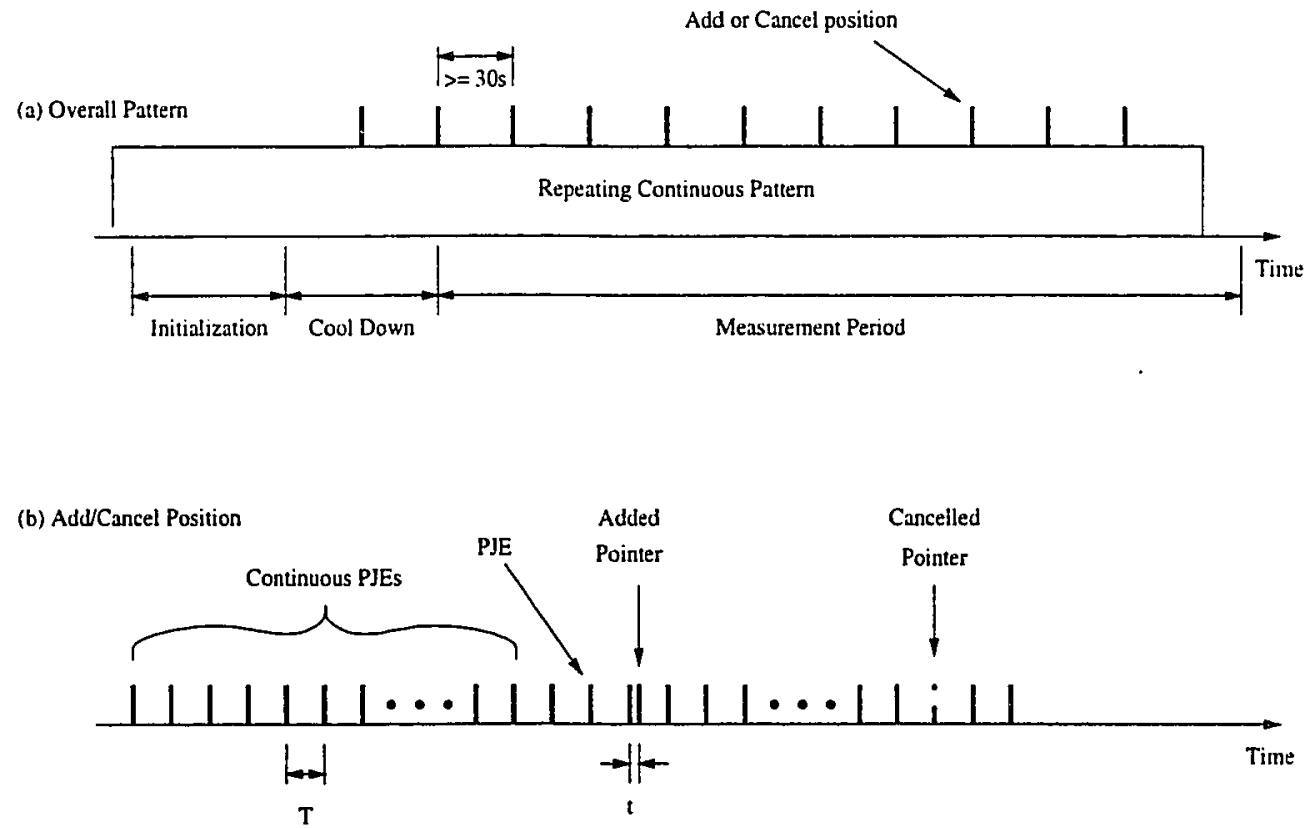

Figure 45. Degraded Mode PJE Test Sequence (Continuous) 


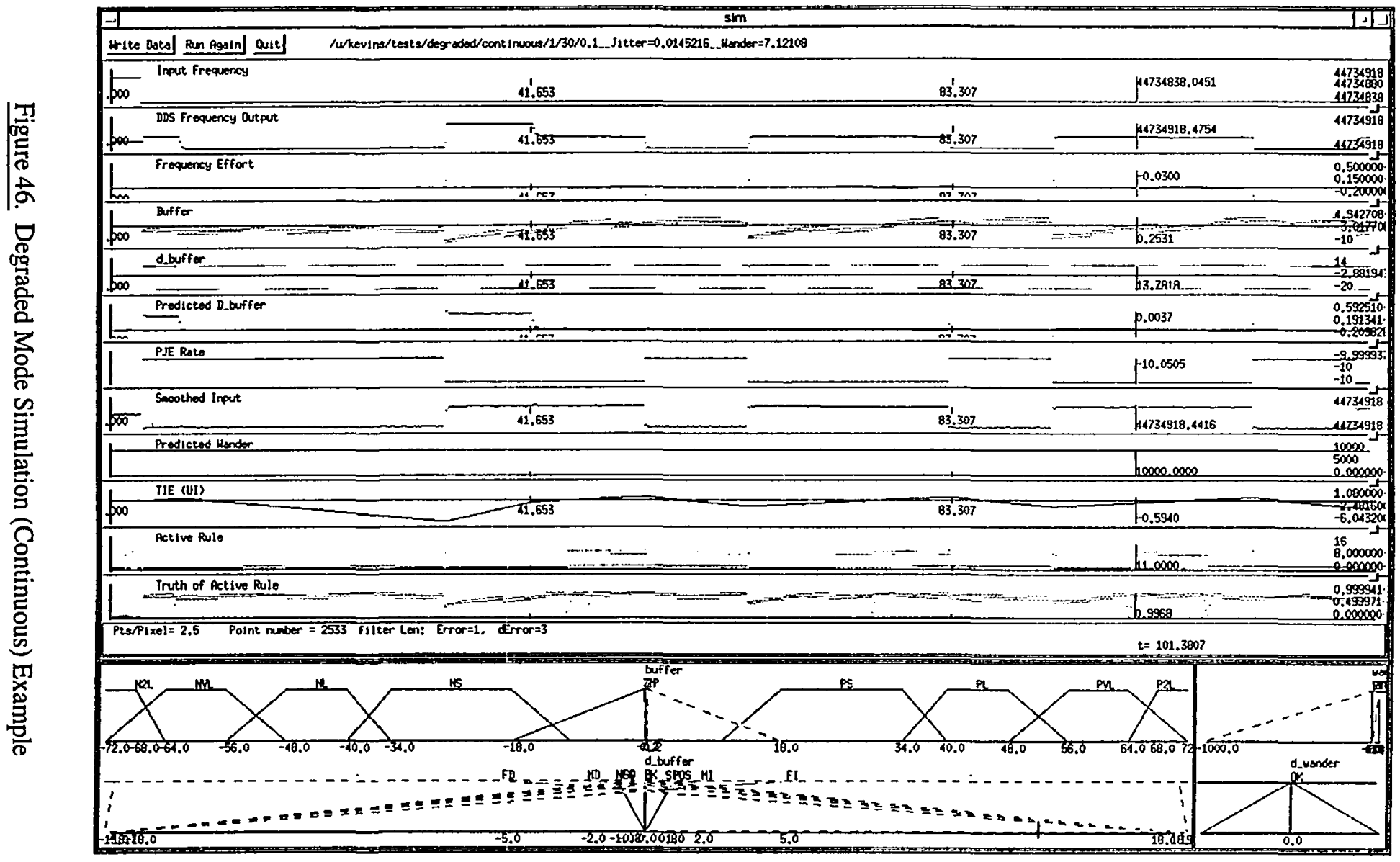


TABLE VIII

Degraded Mode (Continuous Pattern) Jitter

\begin{tabular}{|c|c|c|c|}
\hline \multicolumn{4}{|c|}{ Degraded Mode (Continuous) } \\
\hline Time Between & Time Between. & Jitt & (UI) \\
\hline $\begin{array}{l}\text { Spurious PJEs } \\
\quad(\mathrm{sec})\end{array}$ & $\begin{array}{c}\text { Continuous PJEs } \\
\text { (sec) }\end{array}$ & $\begin{array}{l}\text { Added } \\
\text { PJE }\end{array}$ & $\begin{array}{l}\text { Dropped } \\
\text { PJE }\end{array}$ \\
\hline$\overline{30}$ & 0.034 & 0.0304 & 0.0254 \\
\hline 30 & 0.05 & 0.0162 & 0.0164 \\
\hline 30 & 0.1 & 0.0099 & 0.0101 \\
\hline 30 & 0.2 & 0.0067 & 0.0140 \\
\hline 30 & $\overline{0.4}$ & 0.0063 & 0.0073 \\
\hline 30 & 0.8 & 0.0041 & 0.0108 \\
\hline 30 & I & 0.0184 & 0.0053 \\
\hline 30 & $\overline{2}$ & 0.0186 & 0.0052 \\
\hline 30 & 4 & 0.0036 & 0.0047 \\
\hline 30 & 8 & 0.0031 & 0.0093 \\
\hline 30 & 10 & 0.0283 & 0.0047 \\
\hline 35 & $\overline{0.034}$ & 0.0293 & 0.0263 \\
\hline 35 & 0.05 & 0.0285 & 0.0284 \\
\hline 35 & 0.1 & 0.0091 & 0.0094 \\
\hline 35 & 0.2 & 0.0064 & 0.0155 \\
\hline 35 & 0.4 & 0.0060 & 0.0193 \\
\hline 35 & 0.8 & 0.0046 & 0.0086 \\
\hline 35 & $T$ & 0.0110 & 0.0066 \\
\hline 35 & 2 & 0.0037 & 0.0080 \\
\hline 35 & 4 & 0.0036 & 0.0052 \\
\hline 35 & 8 & 0.0036 & 0.0047 \\
\hline 35 & 10 & 0.0041 & 0.0052 \\
\hline$\overline{40}$ & $\overline{0.034}$ & 0.0285 & 0.0189 \\
\hline 40 & 0.05 & 0.0290 & 0.0189 \\
\hline 40 & 0.1 & 0.0091 & 0.0094 \\
\hline 40 & $\overline{0.2}$ & 0.0064 & 0.0155 \\
\hline 40 & 0.4 & 0.0052 & 0.0108 \\
\hline 40 & 0.8 & 0.0045 & 0.0074 \\
\hline 40 & l & 0.0047 & 0.0069 \\
\hline 40 & 2 & 0.0113 & 0.0050 \\
\hline 40 & 4 & 0.0399 & 0.0167 \\
\hline 40 & 8 & 0.0334 & 0.0179 \\
\hline 40 & 10 & 0.0294 & 0.0037 \\
\hline 60 & 0.034 & 0.0280 & 0.0435 \\
\hline 60 & 0.05 & 0.0288 & 0.0285 \\
\hline
\end{tabular}




\begin{tabular}{|c|c||c|c|}
\hline \multicolumn{4}{|c|}{ Degraded Mode (Continuous) } \\
\hline $\begin{array}{c}\text { Time Between } \\
\text { Spurious PJEs } \\
(\mathrm{sec})\end{array}$ & $\begin{array}{c}\text { Time Between } \\
\text { Continuous PJEs }\end{array}$ & \multicolumn{2}{|c|}{ Jitter (UI) } \\
(sec) & Added & Dropped \\
PJE & PJE \\
\hline 60 & 0.1 & 0.0145 & 0.0096 \\
\hline 60 & 0.2 & 0.0069 & 0.0156 \\
\hline 60 & 0.4 & 0.0066 & 0.0112 \\
\hline 60 & 0.8 & 0.0052 & 0.0144 \\
\hline 60 & 1 & 0.0041 & 0.0066 \\
\hline 60 & 2 & 0.0082 & 0.0050 \\
\hline 60 & 4 & 0.0479 & 0.0042 \\
\hline 60 & 8 & 0.0036 & 0.0047 \\
\hline 50000 & 10 & 0.0336 & 0.0042 \\
\hline 50000 & 0.034 & 0 & 0 \\
\hline 50000 & 0.05 & 0.0004 & 0.0004 \\
\hline 50000 & 0.1 & 0.0004 & 0.0004 \\
\hline 50000 & 0.2 & 0.0004 & 0.0004 \\
\hline 50000 & 0.4 & 0.0004 & 0.0004 \\
\hline 50000 & 0.8 & 0.0009 & 0.0009 \\
\hline 50000 & 1 & 0.0004 & 0.0004 \\
\hline 50000 & 2 & 0.0004 & 0.0004 \\
\hline 50000 & 4 & 0.0004 & 0.0004 \\
\hline 50000 & 8 & 0.0004 & 0.0004 \\
\hline & 10 & 0.0004 & 0.0004 \\
\hline
\end{tabular}

As may be seen from the table, this type of degraded-mode jitter is $8.5 \mathrm{~dB}$ below the 0.9UI specified ( 0.3 for spurious PJEs, and 0.6 for degraded mode).

The standard also states that with $T=\infty$, jitter must be below $0.6 \mathrm{UI}$. This is tested by setting $T=50,000$. As may be seen from Table VIII, the resulting jitter is far below the allowed value of $0.6 \mathrm{UI}$.

The second degraded-mode profile, shown in Figure 48, is called the 87-3 pattern due to the sequence of 87 regularly spaced PJEs followed by three missing PJEs. Superimposed on each pattern of 90 PJE locations is an added or dropped standardmode PJE at an interval of 30 or more seconds $(T)$. The time between PJEs $(t)$ is to span a range from $34 \mathrm{~ms}$ to $10 \mathrm{~s}$. 


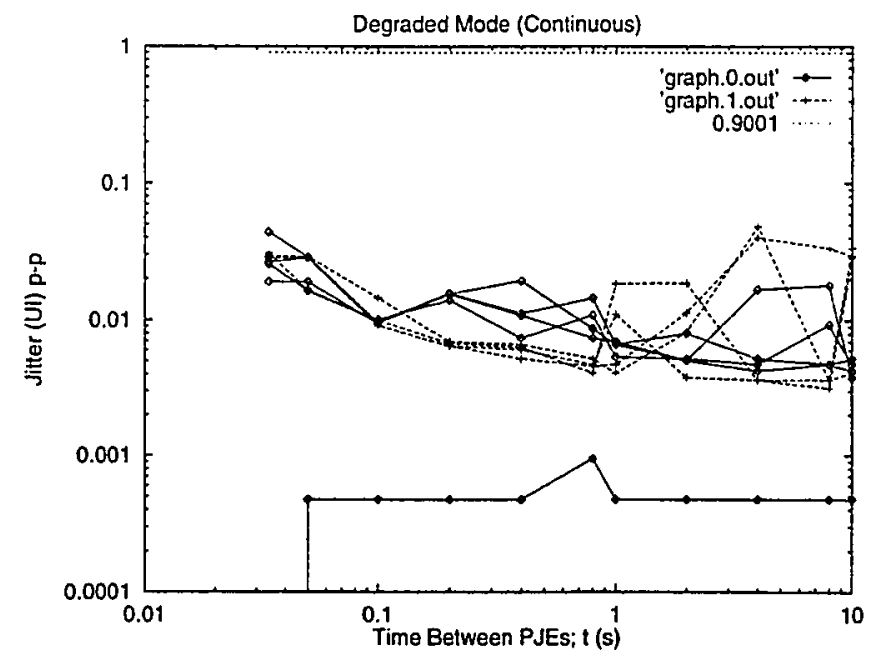

Figure 47. Degraded Continuous Pattern Jitter

The specification requires separate tests for added and dropped pointers. Figure 49 shows a representative $87-3$ simulation. Table IX and Figure 50 show how jitter changes with PJE spacing for both added-PJE and dropped-PJE modes. 

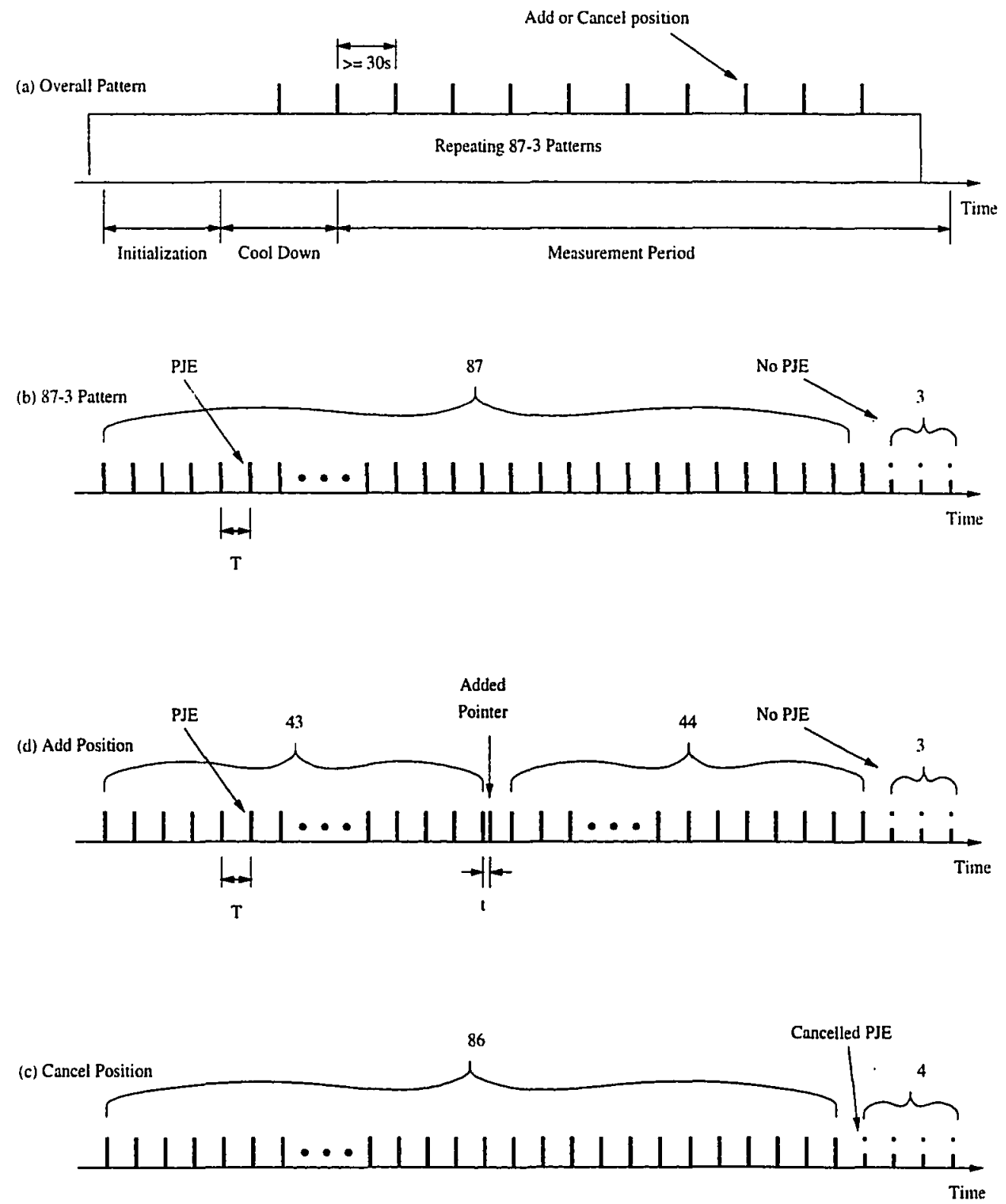

Figure 48. Degraded Mode PJE Test Sequence (Continuous) 


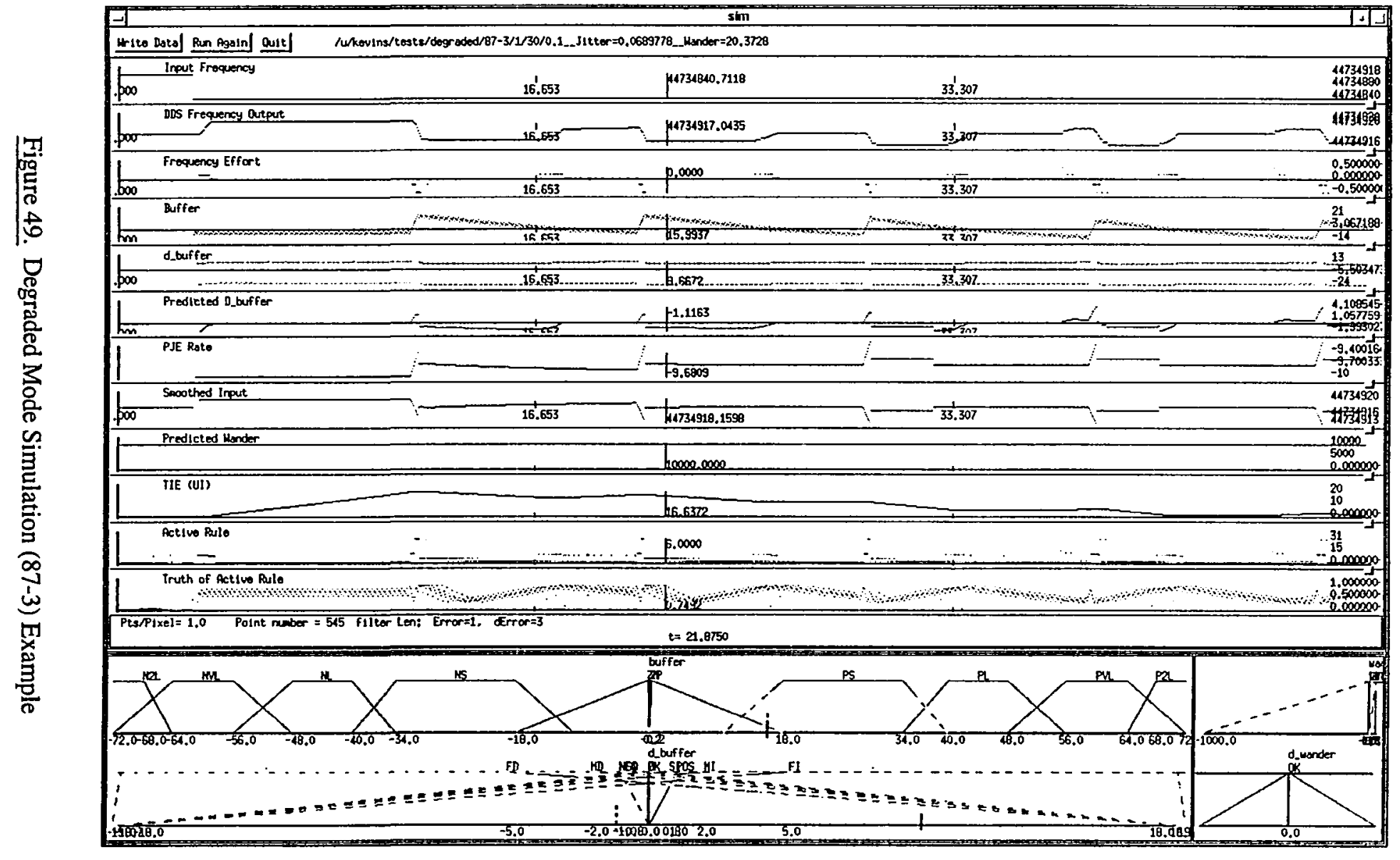




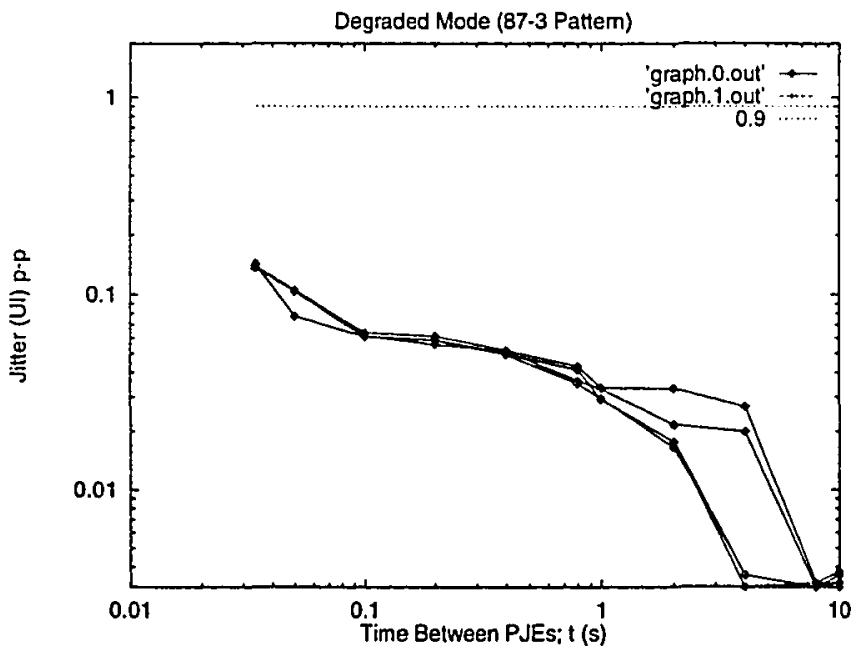

Figure 50. Degraded 87-3 Pattern Jitter

TABLE IX

Degraded Mode (87-3 Pattern) Jitter

\begin{tabular}{|c|c||c|c|}
\hline \multicolumn{4}{|c|}{ Degraded Mode (87-3 Pattern) } \\
\hline $\begin{array}{c}\text { Time Between } \\
\begin{array}{c}\text { Spurious PJEs } \\
\text { (sec) }\end{array}\end{array}$ & $\begin{array}{c}\text { Time Between } \\
\text { Continuous PJEs } \\
(\mathrm{sec})\end{array}$ & $\begin{array}{c}\text { Jitter (UI) } \\
\text { PJE }\end{array}$ & $\begin{array}{c}\text { Dropped } \\
\text { PJE }\end{array}$ \\
\hline \hline 30 & 0.034 & 0.142 & 0.142 \\
\hline 30 & 0.05 & 0.077 & 0.077 \\
\hline 30 & 0.1 & 0.061 & 0.061 \\
\hline 30 & 0.2 & 0.058 & 0.058 \\
\hline 30 & 0.4 & 0.049 & 0.049 \\
\hline 30 & 0.8 & 0.041 & 0.041 \\
\hline 30 & 1 & 0.029 & 0.029 \\
\hline 30 & 2 & 0.017 & 0.017 \\
\hline 30 & 4 & 0.003 & 0.003 \\
\hline 30 & 8 & 0.003 & 0.003 \\
\hline 30 & 10 & 0.003 & 0.003 \\
\hline \hline 35 & 0.034 & 0.139 & 0.139 \\
\hline 35 & 0.05 & 0.103 & 0.103 \\
\hline 35 & 0.1 & 0.060 & 0.060 \\
\hline 35 & 0.2 & 0.058 & 0.058 \\
\hline
\end{tabular}




\begin{tabular}{|c|c|c|c|}
\hline \multicolumn{4}{|c|}{ Degraded Mode (87-3 Pattern) } \\
\hline Time Between & Time Between & & (UI) \\
\hline $\begin{array}{c}\text { Spurious PJEs } \\
\text { (sec) }\end{array}$ & $\begin{array}{l}\text { Continuous PJEs } \\
\text { (sec) }\end{array}$ & $\begin{array}{l}\text { Added } \\
\text { PJE }\end{array}$ & $\begin{array}{l}\text { Dropped } \\
\text { PJE }\end{array}$ \\
\hline 35 & $\overline{0.4}$ & 0.050 & 0.050 \\
\hline 35 & 0.8 & 0.041 & 0.041 \\
\hline 35 & 1 & 0.029 & 0.029 \\
\hline 35 & 2 & 0.017 & 0.017 \\
\hline 35 & 4 & 0.003 & 0.003 \\
\hline 35 & 8 & 0.003 & 0.003 \\
\hline 35 & 10 & 0.003 & 0.003 \\
\hline 40 & 0.034 & 0.135 & 0.135 \\
\hline 40 & 0.05 & 0.103 & 0.103 \\
\hline 40 & 0.1 & 0.061 & 0.061 \\
\hline 40 & 0.2 & 0.055 & 0.055 \\
\hline 40 & 0.4 & 0.051 & 0.051 \\
\hline 40 & 0.8 & 0.036 & 0.036 \\
\hline 40 & 1 & 0.033 & 0.033 \\
\hline 40 & 2 & 0.021 & 0.021 \\
\hline 40 & 4 & 0.019 & 0.019 \\
\hline 40 & 8 & 0.003 & 0.003 \\
\hline 40 & 10 & 0.003 & 0.003 \\
\hline 60 & $\overline{0.034}$ & 0.138 & 0.138 \\
\hline 60 & 0.05 & 0.104 & 0.104 \\
\hline 60 & 0.1 & 0.063 & 0.063 \\
\hline 60 & 0.2 & 0.061 & 0.061 \\
\hline 60 & $\overline{0.4}$ & 0.051 & 0.051 \\
\hline 60 & 0.8 & 0.042 & 0.042 \\
\hline 60 & $T$ & 0.033 & 0.033 \\
\hline 60 & 2 & 0.033 & 0.033 \\
\hline 60 & 4 & 0.026 & 0.026 \\
\hline 60 & 8 & 0.003 & 0.003 \\
\hline 60 & 10 & 0.003 & 0.003 \\
\hline 50000 & 0.034 & 0.140 & 0.140 \\
\hline 50000 & 0.05 & 0.077 & 0.077 \\
\hline 50000 & 0.1 & 0.061 & 0.061 \\
\hline 50000 & $\overline{0.2}$ & 0.058 & 0.058 \\
\hline 50000 & 0.4 & 0.049 & 0.049 \\
\hline 50000 & 0.8 & 0.035 & 0.035 \\
\hline 50000 & T & 0.029 & 0.029 \\
\hline 50000 & 2 & 0.016 & 0.016 \\
\hline 50000 & 4 & 0.003 & 0.003 \\
\hline 50000 & 8 & 0.003 & 0.003 \\
\hline
\end{tabular}




\begin{tabular}{|c|c||c|c|}
\hline \multicolumn{4}{|c|}{ Degraded Mode (87-3 Pattern) } \\
\hline $\begin{array}{c}\text { Time Between } \\
\text { Spurious PJEs } \\
(\mathrm{sec})\end{array}$ & $\begin{array}{c}\text { Time Between } \\
\text { Continuous PJEs } \\
(\mathrm{sec})\end{array}$ & $\begin{array}{c}\text { Added } \\
\text { PJter (UI) }\end{array}$ & $\begin{array}{c}\text { Dropped } \\
\text { PJE }\end{array}$ \\
\hline 50000 & 10 & 0.003 & 0.003 \\
\hline
\end{tabular}

Degraded mode jitter is well below the 0.9UI (0.30UI * 3PJEs) specified. Again, with $T=50000$ the resulting jitter is far below the allowed value of $0.6 \mathrm{UI}$.

6. Jitter Summary

Table X summarizes the peak-to-peak jitter that results from the various PJE input patterns.

TABLE X

Jitter Summary

\begin{tabular}{|l|c|l|c|}
\hline \multicolumn{4}{|c|}{ PJE Jitter Generation } \\
\hline \multicolumn{1}{|c|}{ Sync. Mode } & Allowed & Generated & Attenuation (Db) \\
\hline \hline None (Mapping) & 0.40 & 0.025 & 12.0 \\
\hline Standard & 0.30 & 0.0038 & 19.0 \\
\hline Burst & 0.90 & 0.019 & 16.8 \\
\hline Phase Burst & 0.90 & 0.066 & 11.3 \\
\hline Degraded (Cont., No Add) & 0.60 & 0.00095 & 28.0 \\
\hline Degraded (Cont., Add) & 0.90 & 0.048 & 12.7 \\
\hline Degraded (87-3, No Add) & 0.90 & 0.14 & 8.1 \\
\hline Degraded (87-3, Add) & 0.90 & 0.14 & 8.1 \\
\hline
\end{tabular}

Again, jitter generation is far below the maximum levels for all required PJE tests.

\section{CONSTRAINT-SATISFACTION TESTS}

The second goal of the PKBC - and the most significant contribution of this work - is constraint satisfaction. Since the universal satisfaction of both wander and buffer constraints is not possible, one of two alternatives must be given priority when 
both are in danger of violation. Either the buffer is to be kept from overflowing even if it means MTIE violation, or MTIE is to be kept from violating its specifications even if it means that the buffer will overflow or underflow. These two strategies are investigated in the following two sections.

\section{Wander Satisfied}

The most recent MTIE mask proposal from Bellcore [4] , is shown in Table XI

TABLE XI

Proposed MTIE Mask

\begin{tabular}{|l|l|}
\hline \multicolumn{1}{|c|}{ Observation Time } & \multicolumn{1}{c|}{ MTIE } \\
\hline \multicolumn{1}{|c|}{$(\mathrm{s})$} & \multicolumn{1}{c|}{ (ns) } \\
\hline \hline $\mathrm{S}<0.1$ & N/A (jitter region) \\
\hline $0.1<\mathrm{S}<0.22$ & $4600 * \mathrm{~S}$ \\
\hline $0.22<\mathrm{S}<5100$ & $900+240 * \mathrm{~S}^{1 / 2}$ \\
\hline $5100<\mathrm{S}$ & 18000 \\
\hline
\end{tabular}

and illustrated in Figure 51. Thus we set out to prove that PKBC is capable of satisfying the specified MTIE mask. This was accomplished by simply adding the term:

AND wander IS OK

to the predictive part of each rule as discussed in Section C.5 of Chapter VI. This addition ensures that only those rules with candidate actions which do not violate MTIE are considered. In particular, if a candidate action is predicted to produce an MTIE which is not $\mathrm{OK}$, the above term will evaluate to zero and the rule will not fire.

Since wander is measured in terms of time-to-violation in sample periods, there is no mechanism to keep MTIE from moving arbitrarily close to the envelope. Thus, we provided a simulation parameter which acts to narrow the neck of the envelope artificially at some point in the envelope. This parameter acts as a safety mechanism by generating a predicted MTIE fault when the TIE and envelope lines are about to converge. 


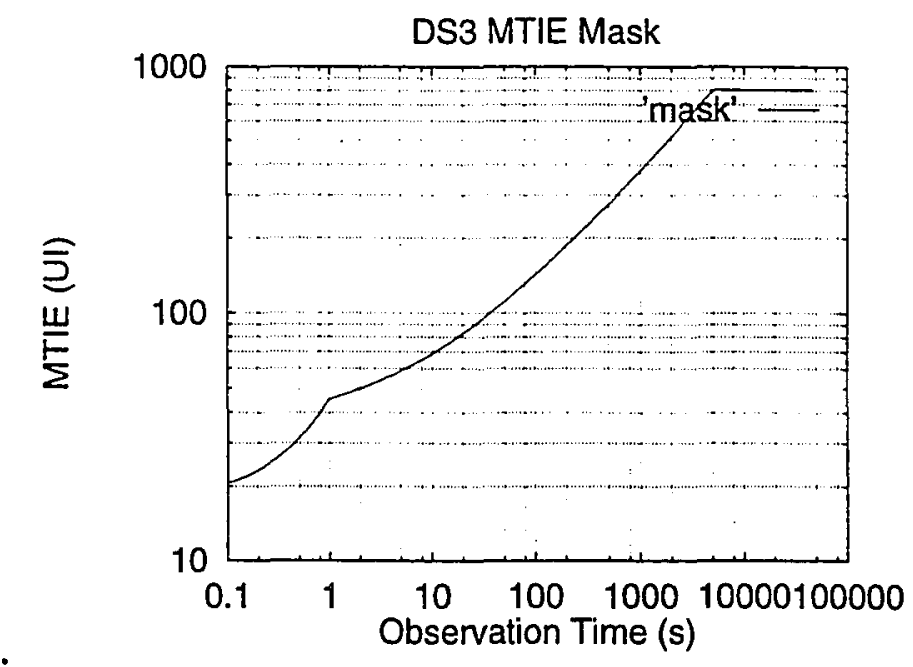

Figure 51. Proposed DS3 Wander Specification

An alternate solution would be to measure the distance to the envelope (a trivial task) and either combine the two measurements into a composite wander-risk-of-violation parameter, or create a new input variable which could be referenced separately by the rules.

The reader will recall that TIE is the integral of the difference between the output frequency and a reference signal at the nominal frequency. Thus, if an input is applied which is different from the reference, the controller initialiy acts to maintain the appropriate buffer level by adjusting the output away from the nominal frequency. The ensuing TIE ramp eventually approaches the MTIE constraint envelope and any actions which would result in an MTIE violation are removed from consideration. The resulting MTIE plot, seen in Figure 52 shows the MTIE mask, the MTIE generated by the controller, and their difference. The generated MTIE approaches the mask but does not cross it - thus satisfying the wander specifications. This graph was precisely what we had hoped to see, and verified our hypothesis regarding the constraint satisfaction capabilities of a PKBC. 


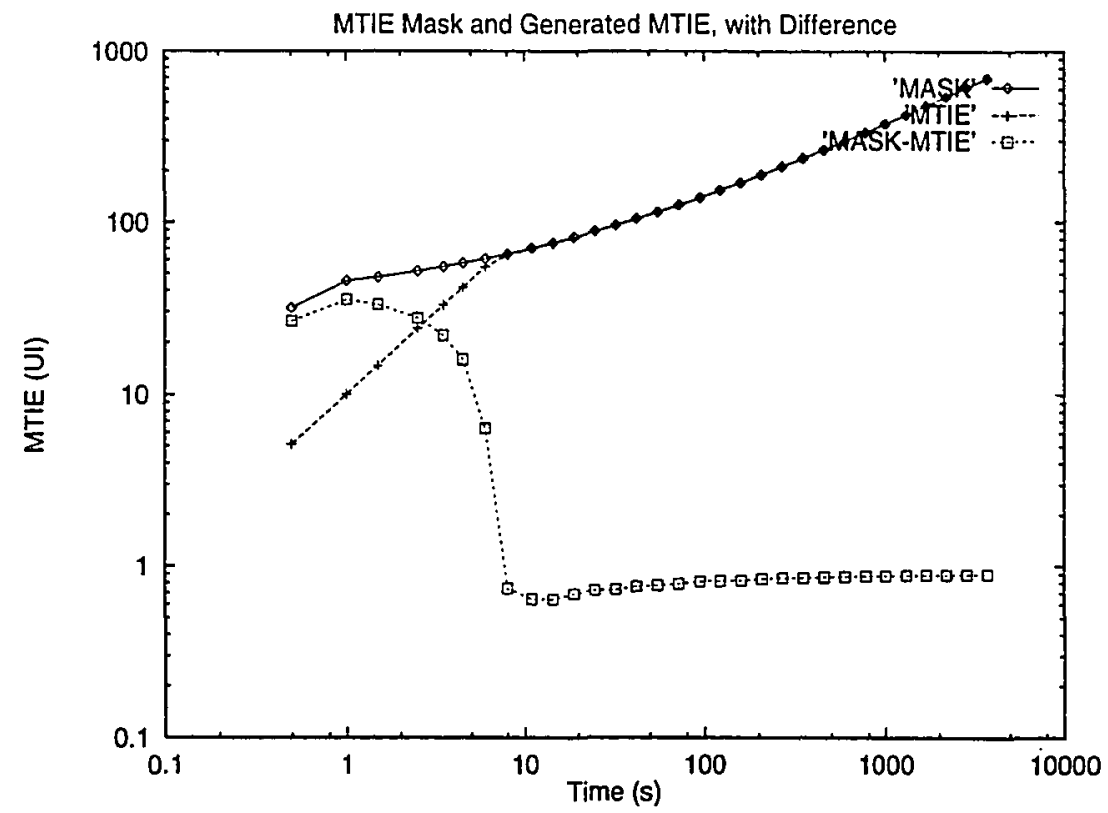

Figure 52. MTIE Satisfaction Plot

Figure 53 shows a similar simulation, this time with the buffer level shown also. Notice that the buffer increases above its desired maximum of 25 when mandated by the MTIE.

Although MTIE is specified in absolute terms, a slight violation is much better than a large violation. So as an added precaution we crafted a set of rules which take effect when the MTIE time-to-violation is negative (TIE is above the ceiling or below the floor of the constraint envelope). These rules take whichever action is predicted to result in the smallest positive wander value (the action which most rapidly moves TIE back inside the envelope).

If the situation occurs where none of the listed candidate actions in the rulebase is predicted to bring MTIE back into compliance, setting the frequency to its nominal value would cause the predicted TIE to become flat. Since the envelope opens 


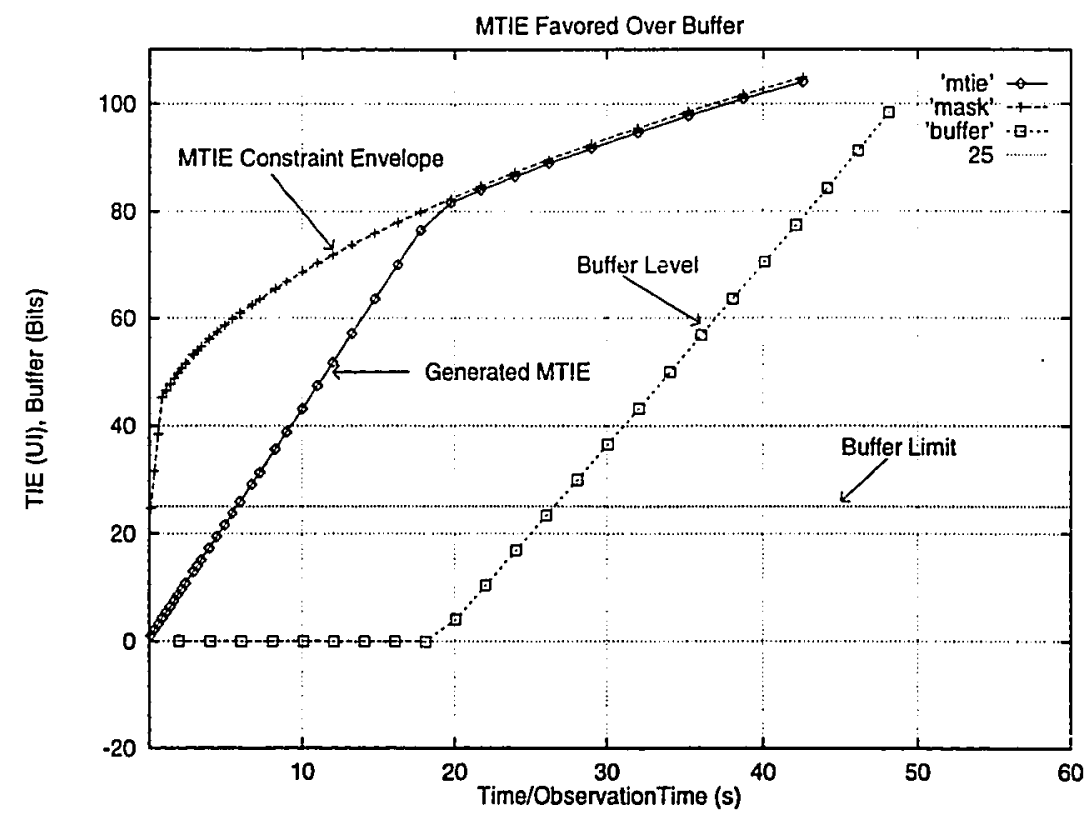

Figure 53. MTIE Satisfaction Plot With Buffer

exponentially, this action would be guaranteed to eventually bring TIE back in compliance. While a commercial application of our desynchronizer might want to include this capability, we have not implemented it.

\section{Buffer Satisfied}

If, instead of maintaining MTIE compliance at all cost, the designer wishes to maintain buffer compliance at all cost, the set of rules described in Section C.6 of Chapter VI is used.

A likely buffer-constraint-satisfaction scenario is now described. An input which is offset from the nominal DS3 frequency is applied to the controller. The controller reacts by tracking the input frequency to maintain the desired buffer level. The TIE that results is a ramp with a slope of $f_{\text {out }}-f_{D S 3}$. Eventually, the MTIE constraint envelope predicts an impending MTIE violation and the standard rules no longer apply - since 
keeping the buffer at the desired level would result in unacceptable wander. Thus, the buffer level begins to ramp. When the $P 2 L$ or $N 2 L$ fuzzy sets become active, the buffer constraint-satisfaction rules begin to apply - resulting in no further increase (or decrease if $N 2 L$ ) of the buffer level. Once both constraints reach their limit, MTIE is violated and the buffer level constraint is maintained as desired. This is illustrated in Figure 54.

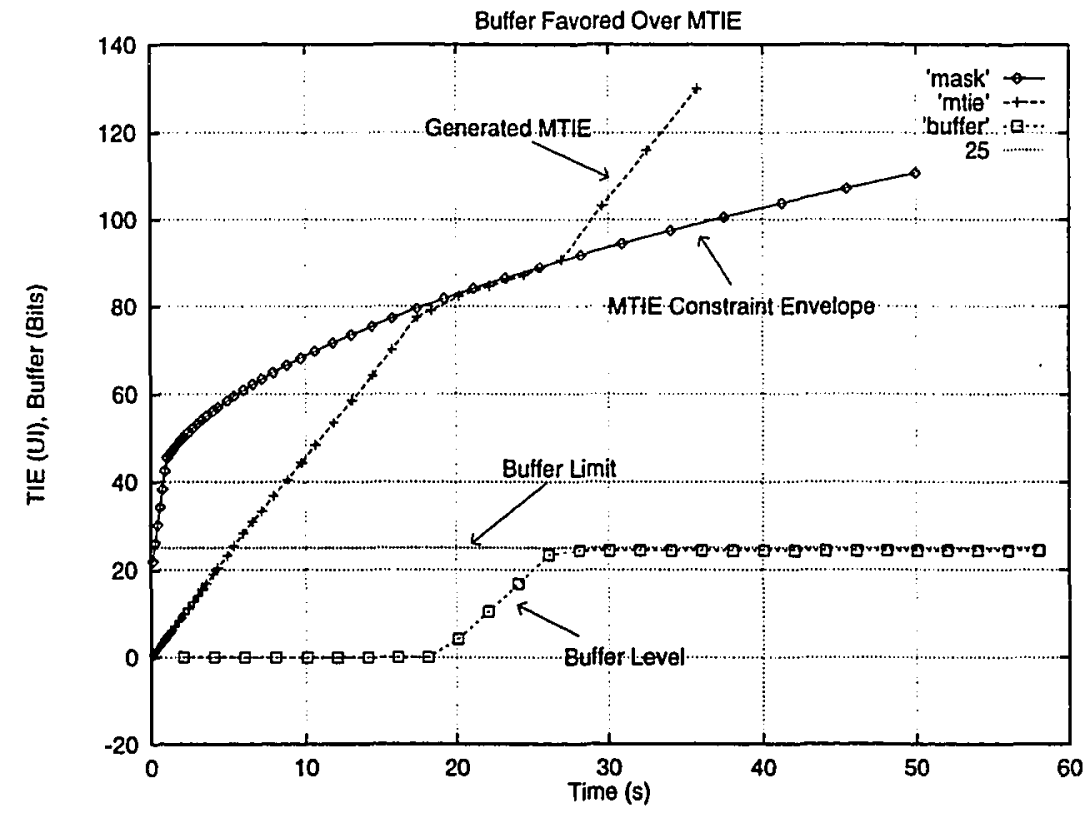

Figure 54. Buffer Satisfaction Plot

Notice that the MTIE plot in this Figure is not a true MTIE calculation, because an MTIE violation at some time $t_{0}$ can cause violations for observation periods of less than $t_{0}$. So, instead of plotting MTIE versus the observation period, MTIE is plotted with respect to simulation so that the form of the graph is the same as in the previous section.

Again, the above plots exemplify the constraint-satisfaction ability of the PKBC. 


\section{CHAPTER IX}

\section{CONCLUSIONS/FUTURE WORK}

\section{A. CONCLUSION}

The need to carry PDH payloads such as the DS3 over a SONET network continues to increase as PDH services gain popularity with corporate customers and SONET gains popularity with public networks.

Our investigation targeted the configuration where a DS3 payload is mapped into an STS-1 envelope and then unmapped and desynchronized at the other end. PJEs within the SONET network were shown to induce both high-frequency jitter and lowfrequency wander in the desynchronized DS3. To ensure compatibility among different equipment manufacturers, the ANSI-accredited T1 committee and Bellcore have developed standards defining acceptable operation. The standard specifies the maximum allowed peak-to-peak jitter for each of several PJE profiles, and wander (MTIE) is specified independently of any particular PJE input pattern. In addition, the desynchronizer must ensure that its elastic store - used to absorb jitter and wander - does not overflow or underflow.

Desynchronizers typically employ a low-pass filter and possibly some non-linear transfer elements to attenuate high-frequency jitter. But the low frequency wander passes below the cutoff frequency of the low-pass filter and can accumulate from network element to network element.

We set out to devise a technique with the following four capabilities: 
- Exhibit the characteristics of a low-pass filter such that the jitter specifications are met implicitly.

- Explicitly satisfy the size constraint of the buffer for as long as the MTIE constraint allows.

- Explicitly satisfy the MTIE constraint for as long as the size constraint of the buffer allows.

- When the above two constraints are in danger of violation, satisfy one at the expense of the other as determined by the designer.

An MTIE violation may result in network instability and/or data errors. A buffer spill results in data errors which would precipitate a retransmission.

As we have shown, a Predictive Knowledge-Based Controller (PKBC) with an expanded rule grammar is a viable alternative to other desynchronizers in terms of jitter attenuation, but stands alone in its ability to dynamically satisfy MTIE and buffer constraints. Our invention of the MTIE constraint envelope (and its efficient calculation) allows the rule-based controller to evaluate the MTIE and buffer level which would result from the application of a particular candidate action. Actions which would needlessly cause constraint violation are removed from consideration automatically. We showed that the computational complexity of the control algorithm is within the reach of modern microprocessors.

To test the constraint satisfaction capabilities of the PKBC, a scenario was presented where the output frequency was adjusted away from the TIE reference frequency to prevent buffer overflow. As a result, TIE began to ramp, causing MTIE to approach the mask. Eventually the controller was forced to slow the increase in TIE by adjusting the output frequency back toward its nominal value. The resulting frequency mismatch between the input and output caused the buffer level to increase. Ultimately, it was 
impossible to simultaneously meet both constraints. For this case we demonstrated the ability of PKBC to sustain either buffer or MTIE compliance. The priority of the constraints was specified in the rules.

In addition to satisfying hard MTIE and buffer constraints, overall jitter generation was very low - less than $0.02 \mathrm{UI}$ for a single PJE - compared to the limit of 0.3UI. The controller was shown to exceed the jitter performance for each pointer adjustment test specified in [3].

Our hypothesis that a PKBC would be capable of good jitter performance in the usual case and MTIE/buffer constraint satisfaction in the limit was thus confirmed.

The effect of our research is two-fold. First, desynchronization equipment which implements a PKBC can be made to generate very small levels of jitter and satisfy the hard constraints whenever possible - leading to better reliability and data integrity in hybrid digital networks. Second, the PKBC has the potential to solve problems in other areas of control. If a model of the process exists and expert knowledge is available for rule generation, this control paradigm has the potential of good performance under normal conditions and constraint satisfaction as a limiting condition.

\section{B. FUTURE WORK}

We have shown the dynamic constraint satisfaction capabilities of a KnowledgeBased Predictive Controller applied to the SONET/DS3 desynchronization problem. A more general study of other applications would serve to broaden understanding of the predictive controller, its rulebase, required model characteristics, and stability.

We extended the standard PKBC by allowing a conditional statement in each rule. Other extensions to the grammar itself might yield other improvements in the designer's ability to express the desired controller characteristics. 
For example, it might be possible to specify a desired outcome in the rule without specifying a candidate action. A single-variable gradient search could then be used to find a suitable action to cause the specified outcome to be realized. This would require an extension to both the grammar and the control algorithm and might also require additional computational resources. Also, additional fuzzy set types would increase the expressive power of the ruleset. In particular, a nonlinear or more general piecewiselinear construct could prove useful in specifying desired behavior more precisely.

With regard to the desynchronization problem, it might be beneficial to study the latency effect of the buffer level compared to other latency effects. If the delay is proportionally significant, it might be possible to modify the rules such that the average number of bits stored in the desynchronizer is reduced without significantly increasing the risk of buffer underflow. While the latency could thereby be reduced, jitter would likely be increased as the controller would need to act more quickly in some situations. Although it is not likely to be reached, [41] defines an upper bound for data latency of $100 \mathrm{~ms}$ where the performance of echo suppression circuitry begins to degrade.

Our rulebase did not explicitly consider the current PJE mode (standard, burst, degraded) when evaluating actions. Instead, we chose a simpler, unified approach. There were two reasons for this. First, this choice simplified the controller, since a PJEmode model would not necessary. Second, we believe that if a single mode-ignorant method can be made to operate acceptably over the broad range of PJE profiles, new or modified PJE profiles will be handled as well. Nonetheless, if PJE mode information were available we expect that jitter performance could of the standard tests could be improved further.

An additional measure of MTIE compliance is a topic worthy of further study. A position measure of the projected TIE within the opening of the MTIE Constraint Envelope would vary more smoothly than the current "time-till-violation" measure 
currently used. A fuzzy region several UI from either envelope boundary would indicate an MTIE hazard to the controller, which could take appropriate action. This could eliminate the need for our technique of artificially narrowing the envelope to maintain a safe distance from the MTIE mask.

Finally, a hardware implementation of our algorithm would allow testing in a real-world environment. Although we believe we have modeled the relevant real-world processes in the simulator, there are always additional factors present when hardware interfaces with a physical environment. We are confident, however, that the research documented here will be shown to be valid and applicable were such a prototype constructed.

We did not employ the phase adjustment capabilities of the DDS. A preliminary investigation found that phase adjustments can be very useful when the buffer level is not changing, and is non-zero. In this case, the phase may simply be adjusted such that the buffer level returns to zero without necessitating a frequency change. 


\section{References}

1. Scott Henderson and Randy Jones, "Synchronizing SONET," Telephony, pp. 27-31 (September 27, 1993).

2. American National Standards Institute (ANSI), "Synchronization Interface Standard," T1.101, American National Standard for Telecommunications (1994).

3. American National Standards Institute (ANSI), For Telecommunicatios - Synchronous Optical Network (SONET) Jitter at Network Interfaces, T1.105.03-1994 (1994).

4. Ron Brown, "Proposal for DS3 Wander Specification at Customer-to-Carrier Interfaces," T1X1.3/96-006, Bellcore (January 1996).

5. George T. Hawley and Frank Nabavi, "Getting to the Pulse on Time," Telephony" (February 1992).

6. D.L. Duttweiler, "Waiting time jitter," Bell System Technical Journal, 51, pp. 165-207 (January 1972).

7. American National Standards Institute (ANSI), "Synchronous Optical Network (SONET) - Basic Description including Multiplex Structure, Rates and Formats," t1x1.5/94-033R7, draft T1.105-199x (1994).

8. Patrick R. Trischitta and Eve L. Varma, Jitter in Digital Transmission Systems, Artech House (1989).

9. Alan V. Oppenheim and Ronald W. Schafer, Discrete-Time Signal Processing, Prentice Hall (1989).

10. Donald F. Stanat and David F. McAllister, Discrete Mathematics in Computer Science, Prentice Hall (1977).

11. R. G. Kusyk, "Techniques for the reduction of DS-1 jitter caused by SONET VT1.5 pointer adjustments"," M.Sc. Thesis, Dept. of Electrical Engineering, University of Alberta (Fall 1990).

12. Hikmet Sari and Georges Karam, "Cancellation of Pointer Adjustment Jitter in SDH Networks," IEEE Transactions on Communications, 42, 12, pp. 3200-3207 (1994).

13. R. F. Bridge and et al., "Jitter Attenuation in T1 Networks," ICC '90 Conf. Rec., 2, pp. 685-689, Atlanta, GA (June 1990).

14. Robert O. Nunn, "SONET Requirements for Jitter Interworking with Existing Networks," IEEE Global Telecommunications Conference, 3, pp. 1501-1505 (1993).

15. R. G. Kusyk, "Analysis of Techniques for the Reduction of Jitter Caused by SONET Pointer Adjustments," IEEE Transactions on Communications, 42, 2/3/4, pp. 2036-2050 (Feb., Mar. 1994).

16. John C. Bellamy, "Digital Network Synchronization," IEEE Communications Magazine, pp. 70-83 (April 1995).

17. Andy Reid, "SONET desynchronizers," ECSA Contribution TIX1.6/89-012 (February 1989).

18. W. D. Grover, T. E. Moore, and J. A. McEachern, "Waiting time jitter reduction by synchronizer stuff threshold modulation," IEEE Globecom 87, 1, pp. 13.7.1-13.7.5 (1987).

19. R. G. Kusyk, T. E. Moore, and W. A. Krzymien, "Spectral analysis of waiting time jitter in the presence of stuff threshold modulation," Electronics Letters, 26, 
8, pp. 526-528 (April 1990).

20. Gianfranco L. Pierobon and Romano P. Valussi, "Jitter Analysis of a Double Modulated Threshold Pulse Stuffing Synchronizer," IEEE Transactions on Communications, 39, 4, pp. 594-602 (April 1991).

21. Lotfi A. Zadeh, "Fuzzy Sets," Information and Control, 8, 3, pp. 338-353 (June 1965).

22. George J. Klir and Tina A. Folger, Fuzzy sets, uncertainty, and information, Prentice-Hall (1988).

23. Ebrahim H. Mamdani, "Application of fuzzy algorithms for contol of simple dynamic plant," Proceedings of the IEE, 121, pp. 1585-1588 (1974).

24. Dimiter Driankov, Hans Hellendoorn, and Michael Reinfrank, An Introduction to Fuzzy Control, Springer-Verlag (1993).

25. Boverie, S. et al., "Fuzzy Logic Control Compared with other Automatic Control Approaches," Proceedings 30th IEEE-CDC Conference on Decision and Control, Brighton, UK (December 11-13, 1991).

26. C.-C. Wong, "Realization of linear outputs by using mixed fuzzy logics," Fuzzy Sets and Systems, 58, 3, p. 329 (Sep. 24, 1993).

27. Bart Kosko, Neural Networks and Fuzzy Systems : A Dynamical Systems Approach to Machine Intelligence, Prentice-Hall (1992).

28. Adaptive Logic, "Single-Chip Fuzzy Logic NLX230 Information Sheet," 411 Central Park Drive, Sanford, FL 32771 (1995).

29. S. Yasunobu and S. Miyamoto, "Automatic Train Operation System by Predictive Fuzzy Control," Industrial Applications of Fuzzy Control (M. Sugeno ed.), pp. 1-18, North-Holland (1985).

30. S. Yasunobu, S. Miyamoto, T. Takaoka, and H. Oshima, "Application of Predictive Fuzzy Control to Automatic Train Operation Controller," Proceedings of the 1984 International Conference on Industrial Electronics, Contrl and Instrumentation, 2, pp. 657-662 (1984).

31. Toshiro Terano, Kiyoji Asai, and Michio Sugeno, Applied Fuzzy Systems, Academic Press (AP) Professional (1994).

32. Seiji Yasunobu and Yasuhito Murai, "Predictive Fuzzy Control and Parking Control," Proceedings of the American Control Conference (1995).

33. M. Maeda, M. Shimakawa, and S. Murakami, "Predictive fuzzy control of an autonomous mobile robot with forecase learning function," Fuzzy Sets and Systems, 72, 1, p. 51 (May 26, 1995).

34. Kumpati S. Narendra and Kannan Parthasarathy, "Identification and Control of Dynamical Systems Using Neural Networks," IEEE Transactions on Neural Networks, 1, 1, pp. 5-27 (March 1990).

35. Ye. V. Zilbert and M. N. Koltonov, "Precision digital phase discriminator for a digital network synchronization system," Telecommunications and Radio Engineering, 46, 10, pp. 37-43 (Oct 1991).

36. M. E. Lesk, "Lex - A Lexical Analyzer Generator," Computing Science Technical Report, 39, Bell Laboratories, Murray Hill, New Jersey (October 1975).

37. S. C. Johnson, "Yacc: Yet Another Compiler Compiler," Computing Science Technical Report, 32, Bell Laboratories, Murray Hill, NJ 07974 (1975). 
38. Witold Pedrycz, "Why triangular membership functions?" Fuzzy sets and systems, 64, pp. 21-30 (1994).

39. Margret A. Ellis and Bjarne Stroustrup, The Annotated C++ Reference Manual, Addison-Wesly (1990).

40. Alfred V. Aho, Brian W. Kernighan, and Peter J. Weinberger, The AWK Programming Language, Addison-Wesley (1988).

41. Peter Kartaschoff, "Synchronization in Digital Communications Networks," Proceedings of the IEEE, 79, 7, pp. 1019-1028 (July 1991). 